\title{
Ingredients for solar-like systems: protostar IRAS 16293-2422 B versuS comet 67P/Churyumov-Gerasimenko
}

\author{
Maria N. Drozdovskaya ${ }^{\oplus},{ }^{1 \star}$ Ewine F. van Dishoeck, ${ }^{2,3}$ Martin Rubin ${ }^{\oplus},{ }^{4}$ \\ Jes K. Jørgensen ${ }^{5}$ and Kathrin Altwegg ${ }^{\oplus 4}$ \\ ${ }^{1}$ Center for Space and Habitability, Universität Bern, Sidlerstrasse 5, CH-3012 Bern, Switzerland \\ ${ }^{2}$ Leiden Observatory, Leiden University, PO Box 9513, NL-2300 RA Leiden, The Netherlands \\ ${ }^{3}$ Max-Planck-Institut für Extraterrestrische Physik, Giessenbachstrasse 1, D-85748 Garching, Germany \\ ${ }^{4}$ Physikalisches Institut, Universität Bern, Sidlerstrasse 5, CH-3012 Bern, Switzerland \\ ${ }^{5}$ Niels Bohr Institute \& Centre for Star and Planet Formation, University of Copenhagen, Øster Voldgade 5-7, DK-1350 Copenhagen K., Denmark
}

Accepted 2019 August 29. Received 2019 August 27; in original form 2019 May 27

\begin{abstract}
Our modern day Solar System has $4.6 \times 10^{9} \mathrm{yr}$ of evolution behind it with just a few relics of its birth conditions remaining. Comets are thought to be some of the most pristine tracers of the initial ingredients that were combined to produce the Earth and the other planets. Other low-mass protostars may be analogous to our proto-Sun and hence, could be used to study the building blocks necessary to form Solar-like systems. This study tests this idea on the basis of new high sensitivity, high spatial resolution ALMA data on the protoplanetary disc-scales ( $\sim 70 \mathrm{au}$ ) of IRAS 16293-2422 and the bulk composition of comet 67P/ChuryumovGerasimenko, as determined for the first time with the unique in situ monitoring carried out by Rosetta. The comparative analysis of the observations from the Protostellar Interferometric Line Survey (PILS) and the measurements made with Rosetta Orbiter Spectrometer for Ion and Neutral Analysis (ROSINA) shows that the relative abundances of CHO-, N-, and S-bearing molecules correlate, with some scatter, between protostellar and cometary data. A tentative correlation is seen for the first time for P- and Cl-bearing compounds. The results imply that the volatile composition of cometesimals and planetesimals is partially inherited from the preand protostellar phases of evolution.
\end{abstract}

Key words: astrochemistry-comets: general-comets: individual: 67P/ChuryumovGerasimenko-stars: protostars - ISM: molecules.

\section{INTRODUCTION}

Frozen volatile molecules are found in our Solar System in cold distant regions from the Sun or within bodies sufficiently large to shelter the ices from thermal desorption. Consequently, this includes large $\left(<10^{3} \mathrm{~km}\right)$ icy moons such as Europa or Enceladus, and small $(\sim \mathrm{km}$-sized) distant comets. As our mature Solar System is devoid of gas on disc-scales, ices still present today must have been formed during earlier evolutionary phases of our system when gases were still available for adsorption. This implies that cometary ices are made from the gases and ices found in the protoplanetary disc and the prestellar core (Greenberg \& Li 1999; Ehrenfreund \& Charnley 2000). Prestellar ices may be entirely inherited by comets (i.e. pristine), or may be partially or entirely modified en route to and inside the disc and into the comets (i.e. partial or full reset). Recent

^E-mail: maria.drozdovskaya@csh.unibe.ch measurements of a very high ratio of 17 for $\mathrm{D}_{2} \mathrm{O} / \mathrm{HDO}$ relative to $\mathrm{HDO} / \mathrm{H}_{2} \mathrm{O}$ in comparison to the statistically expected value of 0.25 (Altwegg et al. 2017a) on comet 67P/Churyumov-Gerasimenko, hereafter $67 \mathrm{P} / \mathrm{C}-\mathrm{G}$, corroborate the pristinity of cometary water ice. The abundance of highly volatiles species, such as $\mathrm{CO}, \mathrm{N}_{2}$, and noble gases (Rubin et al. 2018) on 67P/C-G rejects the possibility of full reset, as the forming disc is unlikely to ever be sufficiently cold to re-adsorb these molecules. Comets and other icy planetesimals have been postulated to bring water and the ingredients for life to our planet due to their significant late-time dynamics. Hence, understanding their composition and origins may shed light on the emergence of life on Earth, its ubiquity on other planets, and in extrasolar systems (as reviewed in A'Hearn 2011; Mumma \& Charnley 2011; Bockelée-Morvan et al. 2015a).

The formation of a protostar and its protoplanetary disc is governed by gravitational collapse (Shu, Adams \& Lizano 1987). This process is coupled with grain-growth mechanisms transforming $0.1 \mu \mathrm{m}$-sized dust grains found in prestellar cores to mm-sized 
dust particles seen in discs. Disc-scale gas and dust hydrodynamic processes subsequently assemble $\mathrm{m}$-sized planetesimals. Cometary bodies may be a normal by-product of planet formation across the disc in the form of remnant building blocks or even primordial rubble piles composed of their own building blocks (A'Hearn 2011; Davidsson et al. 2016). Once the icy dust/rocks are assembled into a $\mathrm{km}$-sized body, it is unlikely to be significantly thermally processed. Most recent calculations suggest that even a perihelion passage of a comet at 1.2 au from the Sun will only heat the outermost few tens of $\mathrm{cm}$ (at least for the morphology of 67P/C-G; Schloerb et al. 2015; Capria et al. 2017). Meanwhile, non-catastrophic collisions are also unlikely to generate sufficient energy for significant heating (e.g. Jutzi et al. 2017; Jutzi \& Benz 2017; Schwartz et al. 2018). Hence, bulk cometary ices very likely closely resemble disc and prestellar ices and gases (Pontoppidan et al. 2014). Consequently, cometary bulk composition may yield information about the ingredients for Solar-like systems.

For a long time, the Oort cloud comet C/1995 O1 (Hale-Bopp) was the best studied cometary body thanks to its high brightness (total visual magnitude of $\sim 10.5$ at a heliocentric distance of $7 \mathrm{au}$ ). Bockelée-Morvan et al. (2000) used ground-based sub-mm (80$370 \mathrm{GHz}$ ) facilities to study the chemical composition of HaleBopp's coma between 1997 February and April for heliocentric distances in the 0.91-1.2 au range close to its perihelion on 1997 April 1. These data were used to infer a strong correlation between Hale-Bopp's abundances of CHO- and N-bearing molecules and those derived from interstellar medium (ISM) observations on envelope- or cloud-scales (thousands of au). In contrast, the Sbearing species showed a large scatter. At the time, it was unclear whether such trends would persist for other comets. Now, the Rosetta mission has yielded unprecedented detail and wealth of information on another Jupiter-family comet, 67P/C-G (as reviewed in Altwegg, Balsiger \& Fuselier 2019). The mission accompanied and continuously monitored the comet with its suite of instruments for more than $2 \mathrm{yr}$ pre- and post- its 2015 August 12 perihelion for heliocentric distances starting at 4.4 au down to 1.24 au and back out to 3.8 au (distances of aphelion and perihelion are 5.683 and $1.243 \mathrm{au}$, respectively).

The target of the Rosetta mission, comet $67 \mathrm{P} / \mathrm{C}-\mathrm{G}$, is composed of two lobes that are $4.1 \times 3.5 \times 1.6$ and $2.5 \times 2.1 \times 1.6 \mathrm{~km}$ in size (Jorda et al. 2016). Thanks to the continuous monitoring of the target by the orbiter, it was realized that there is much variability in the outgassing of ices hidden underneath the surface, which has to do with seasonal and diurnal variations as probed with Rosetta Orbiter Spectrometer for Ion and Neutral Analysis (ROSINA), Visible and Infrared Thermal Imaging Spectrometer (VIRTIS), Microwave Instrument for the Rosetta Orbiter (MIRO) and Optical, Spectroscopic, and Infrared Remote Imaging System (OSIRIS) instruments aboard Rosetta (e.g. Biver et al. 2015; De Sanctis et al. 2015; Hässig et al. 2015; Lee et al. 2015; LuspayKuti et al. 2015; Bockelée-Morvan et al. 2015b; Barucci et al. 2016; Bockelée-Morvan et al. 2016; Fornasier et al. 2016; Hansen et al. 2016; Migliorini et al. 2016; Filacchione et al. 2016a,b; Gasc et al. 2017; Marshall et al. 2017; Filacchione et al. 2019). The bilobate geometry of the nucleus and the associated self-shielding, its changing rotational period, backfall of granular material, shortlived outbursts, active sinkhole pits, and orbital trajectory change the irradiance of its surface during a single apparition (Keller et al. 2015; Vincent et al. 2015, 2016; Feldman et al. 2016; Keller et al. 2017; Kramer et al. 2018), but also in the long term upon repeated approaches to the Sun. Nevertheless, it is possible to extract bulk abundances of the interior ices upon careful data analysis (e.g.
Calmonte et al. 2016) and to peek at them on special occasions such as cliff collapses (Pajola et al. 2017). The nucleus of 67P/C-G is thought to be homogeneous based on Comet Nucleus Sounding Experiment by Radiowave Transmission (CONSERT) and Radio Science Investigation (RSI) experiment measurements (Kofman et al. 2015; Pätzold et al. 2016). It seems likely that its shape stems from a merger of two distinct objects (e.g. Jutzi \& Asphaug 2015; Massironi et al. 2015; Matonti et al. 2019).

ISM astrochemical studies have also been profiting from new facilities capable of high spatial resolution observations, allowing Solar System scales $(<100 \mathrm{au})$ to be probed for the first time in young star-forming regions (e.g. ALMA Partnership et al. 2015; Ansdell et al. 2016). The closest Solar-like system that is still in its infant embedded phase of formation, the low-mass binary IRAS 16293-2422, has been the target of many observational campaigns over the last few decades with single-dish and interferometric facilities (see Jørgensen et al. 2016 for a review). The system still finds itself in the earliest, gas-rich, embedded phase of star formation corresponding to the suspected time of cometesimal formation. Now, with ALMA, accurate abundances of several tens of molecules can be determined in the disc-like structures found in this puerile system. This allows the volatiles being incorporated into comets and planetesimals in IRAS 16293-2422 to be directly compared to those found in our Solar System on analogous spatial scales. Early hints for the interstellar - cometary relation in the context of this protostellar source and comet Hale-Bopp have been investigated by Schöier et al. (2002), but were unable to access the most inner disc-forming regions based on data from facilities less powerful in comparison to ALMA.

IRAS 16293-2422 is the closest protostellar low-mass system that has been well-characterized physically and chemically (Jørgensen et al. 2016). It is composed of two deeply embedded protostars, $\mathrm{A}$ and $\mathrm{B}$, at a short distance of $141 \mathrm{pc}$ (Dzib et al. 2018) with a projected separation of 5".3 (747 au). The masses and luminosities have been estimated to be on the order of $18 \mathrm{~L}_{\odot}, 1.0 \mathrm{M}_{\odot}$ for source $\mathrm{A}$ and $3 \mathrm{~L}_{\odot}, 0.1 \mathrm{M}_{\odot}$ for source $\mathrm{B}$, based on previous observations and theoretical models (Jacobsen et al. 2018). The total amount of mass encompassed by the circumbinary envelope of $\sim 50 \mathrm{arcsec}$ in size is $\sim 4 \mathrm{M}_{\odot}$ (Jacobsen et al. 2018). High spatial resolution observations with ALMA have resolved the scales of the two individual discs, i.e. on scales of a few tens of au. The data suggest that the disc around source A is nearly edge-on, while that around source B is face-on (e.g. Pineda et al. 2012; Zapata et al. 2013). This has also been independently supported via dust continuum polarization studies (e.g. Liu et al. 2018; Sadavoy et al. 2018). The outer dust disc radius of B is suggested to be about 30-56 au (Rodríguez et al. 2005; Zapata et al. 2013; Hernández-Gómez et al. 2019a). The velocity gradient across the 'disc'-domain of source B (Zapata et al. 2013) is much shallower than that across A (Girart et al. 2014). It has so-far not been possible to determine the relative ages of the A and B sources using signatures of infall and chemical differentiation (e.g. Chandler et al. 2005; Zapata et al. 2013; Calcutt et al. 2018b; Rivilla et al. 2019; van der Wiel et al. 2019). It is unlikely for there to be a drastic age gap, as the two protostars are part of a binary system and are both still undergoing gravitational collapse.

To explore the hypothesis of close ties between cometary and protostellar chemical inventories, it is necessary to assume that all low-mass systems evolve analogously. In this paper, comet $67 \mathrm{P} / \mathrm{C}-\mathrm{G}$ will be considered as a representative probe of the bulk cometary ices; and IRAS 16293-2422 B will assume the role of a Solar-like embedded system. The goal of this paper is to compare the chemical inventories of these two targets and thereby test 
the chemical links that may or may not exist between cometary and interstellar volatiles. This work showcases the synergy of the powerful capabilities of Rosetta and ALMA. Section 2 describes the data that are used in this paper to obtain the results presented in Section 3. The implications of the findings are presented in Section 4 and the conclusions are summarized in Section 5.

\section{METHODS}

\section{$2.167 \mathrm{P} / \mathrm{C}-\mathrm{G}$}

The data on comet $67 \mathrm{P} / \mathrm{C}-\mathrm{G}$ analysed in this work stem from the ROSINA instrument suite aboard the orbiter, which measures the gases stemming from the comet at the distance of the orbiter from the comet surface. This reduces the uncertainties stemming from photodissociation rates, which are required to correct for photodissociation of molecules in the coma when observing with ground-based facilities (Bockelée-Morvan et al. 2000). The ROSINA Double Focusing Mass Spectrometer (DFMS) has a highmass resolution $(m / \Delta m=3000$ on mass/charge of $28 \mathrm{u} / \mathrm{e}$ at the 1 percent peak height) and the ROSINA Reflection-type TimeOf-Flight (RTOF) mass spectrometer has a wide mass range (1$1000 \mathrm{u} / \mathrm{e})$, allowing unambiguous identification of small and large molecules (Balsiger et al. 2007).

Here, the averages of the measurements obtained between 2015 May 22 and June 2 are used as bulk abundances. During this period, the orbiter was at distances in the $100-200 \mathrm{~km}$ range from the comet surface. This specific 2015 May time frame is ideal for measuring the bulk volatile content (Calmonte et al. 2016; Altwegg et al. 2019). It starts at the final pre-2015-perihelion equinox of the comet and ends prior to the coma becoming significantly polluted with dust as a result of higher activity closer to the Sun. During this period, the Southern hemisphere began experiencing the summer season (which is short, yet intense, in comparison to the summer experienced by the Northern hemisphere). This hemisphere is thought to be less covered by the resettled dust from earlier perihelia (Keller et al. 2017). Finally, during this time, 67P/C$\mathrm{G}$ was within $\sim 2$ au from the Sun, thus subjecting it to surface temperatures well above those required for thermal desorption of water. This implies that almost all the volatiles were sublimating at this time, unlike at larger distances when the volatility of molecules affects their observed desorption patterns. Even closer to the Sun, coma abundances become affected by outbursts, which appear to be powered by $\mathrm{CO}_{2}$ and in turn, are less representative of the bulk interior.

\subsection{IRAS 16293-2422}

In this work, the majority of observational data on IRAS 162932422 stem from the large unbiased Protostellar Interferometric Line Survey (PILS; ${ }^{1}$ project-id: 2013.1.00278.S, PI: Jes K. Jørgensen) carried out with ALMA in the $329-363 \mathrm{GHz}$ frequency range (Band 7) during Cycle 2 observations, supplemented with ALMA observations in Bands $3(\sim 100 \mathrm{GHz})$ and $6(\sim 230 \mathrm{GHz})$ carried out during Cycle 1 (project-id: 2012.1.00712.S, PI: Jes K. Jørgensen; Jørgensen et al. 2016). This data set represents the most complete spectral characterization of the source on (almost) identical spatial scales (the beam size does vary slightly with frequency across the large range observed). PILS was carried out at a spectral resolution

\footnotetext{
${ }^{1}$ http://youngstars.nbi.dk/PILS/
}

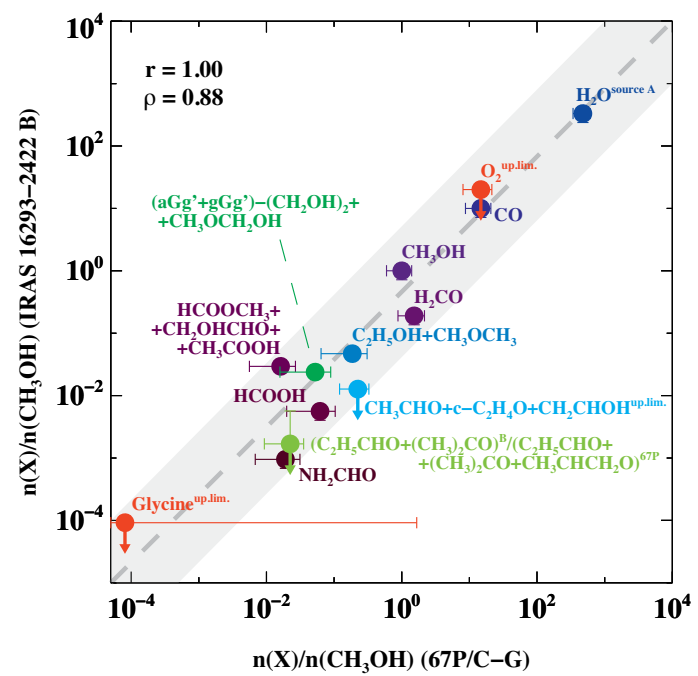

Figure 1. The abundance of CHO-bearing molecules relative to methanol detected towards the one-beam offset position from IRAS 16293-2422 B versus that measured in $67 \mathrm{P} / \mathrm{C}-\mathrm{G}$. Each molecule is marked with a unique colour. The shaded region corresponds to an order of magnitude scatter about the linear correlation. The Pearson $(r)$ and Spearman $(\rho)$ correlation coefficients are given in the upper left corner. 'up.lim.' indicates the values that are protostellar upper limits; and 'Source A' indicates the value that is an estimate based on IRAS 16293-2422 A.

of $0.2 \mathrm{~km} \mathrm{~s}^{-1}(0.244 \mathrm{MHz})$ and restored with a uniform circular beam of 0.5 . The data from the main array of $12 \mathrm{~m}$ dishes are combined with data from the Atacama Compact Array (ACA) of $7 \mathrm{~m}$ dishes, hence, resulting in the largest recoverable size of 13 arcsec. This implies that the PILS data set can be used to study the emission on the scale of the individual envelopes of the two protostars $(\sim 1-3 \operatorname{arcsec})$ and on the scale of their discs $(<1$ arcsec). The main position analysed in this work is a one-beam offset position from source B in the SW direction, which optimizes high densities (hence, boosting column densities of the least abundant molecules), while avoiding the self-absorption and dust absorption at the highest available densities found on-source (e.g. Coutens et al. 2016; Ligterink et al. 2017; Lykke et al. 2017; Calcutt et al. 2018b; Drozdovskaya et al. 2018; Manigand et al. 2019). Relative abundances at the half-beam offset position from source B in the same direction are similar to those at the full-beam offset position, but with a factor of 2 higher column density (Jørgensen et al. 2018). The offset positions in terms of the continuum and molecular emission distributions have been shown in previous publications (e.g. fig. 7 of Jørgensen et al. 2016; fig. 2 of Jørgensen et al. 2018; or fig. 1 of Drozdovskaya et al. 2018). The narrower line widths associated with the dynamical structure near source B reduce line blending. Taking the known physical structure of source B and its disc into account, at 0.'5 ( 70 au) from the source, the observations probe the material entering the protoplanetary disc, especially, since infalling velocity signatures are seen (Pineda et al. 2012).

\section{RESULTS}

\subsection{Correlations between IRAS 16293-2422 B and 67P/Churyumov-Gerasimenko}

Figs 1-4 show log-log plots of the observed relative abundances of 67P/C-G and IRAS 16293-2422 B used to search for correlations between bulk cometary volatiles and protoplanetary disc-materials. 


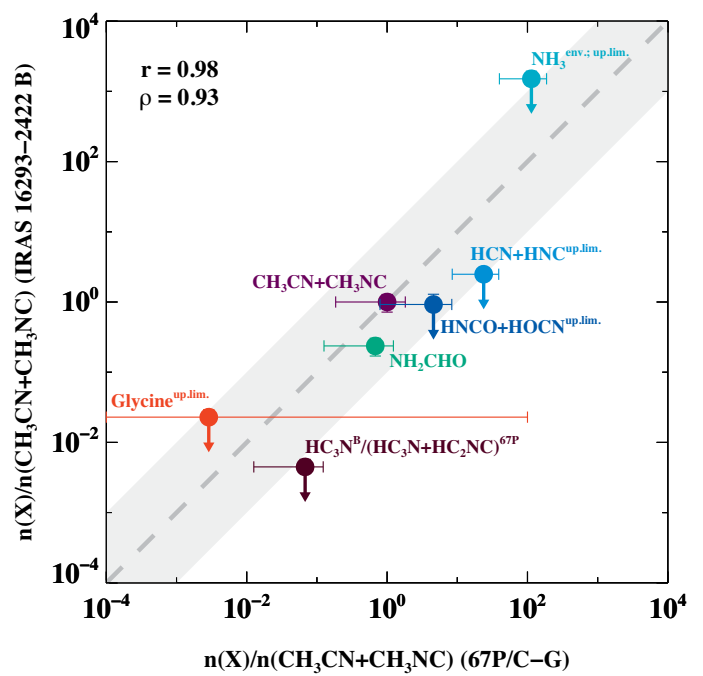

Figure 2. The abundance of N-bearing molecules relative to methyl cyanide detected towards the one-beam offset position from IRAS 16293-2422 B versus that measured in $67 \mathrm{P} / \mathrm{C}-\mathrm{G}$. Each molecule is marked with a unique colour. The shaded region corresponds to an order of magnitude scatter about the linear correlation. The Pearson $(r)$ and Spearman's $(\rho)$ correlation coefficients are given in the upper-hand corner. 'up.lim.' indicates the values that are protostellar upper limits; and 'env.' indicates the value that is an estimate based on the circumbinary envelope.

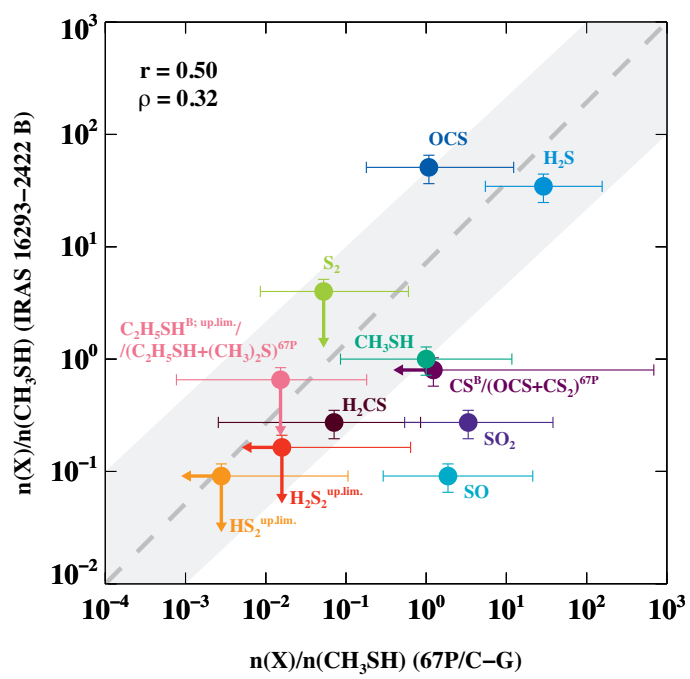

Figure 3. The abundance of S-bearing molecules relative to methyl mercaptan detected towards the one-beam offset position from IRAS 16293-2422 B versus that measured in $67 \mathrm{P} / \mathrm{C}-\mathrm{G}$. Each molecule is marked with a unique colour. The shaded region corresponds to an order of magnitude scatter about the linear correlation. The Pearson $(r)$ and Spearman's $(\rho)$ correlation coefficients are given in the upper left-hand corner. 'up.lim.' indicates the values that are protostellar upper limits.

The volatiles have been partitioned into chemical families based on the elements that they carry. The reference species for computing the relative abundances differ per chemical family and have been chosen based on the interstellar chemical pathways to form such chemically related molecules. The majority of cometary data points represent bulk abundances as derived from ROSINA measurements, as explained in Section 2.1 and published in Rubin et al. (2019). The protostellar values represent the material entering the protoplanetary

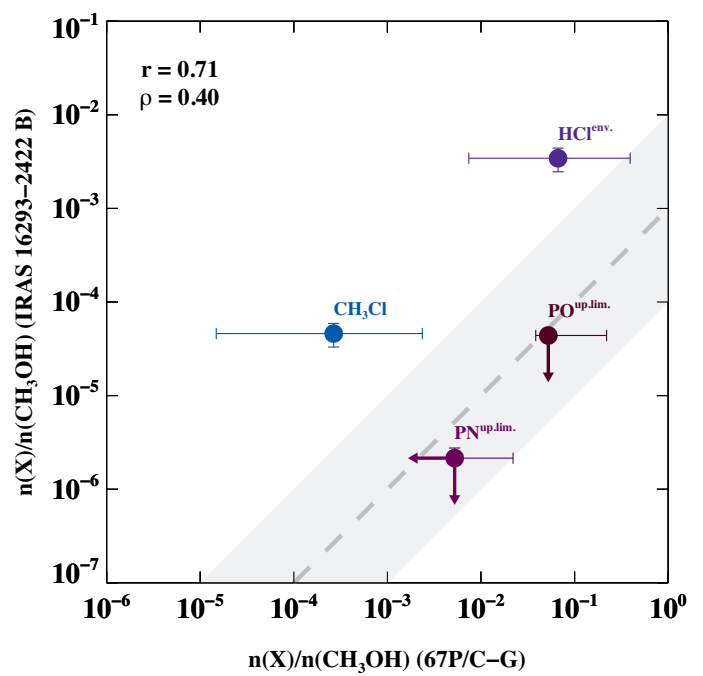

Figure 4. The abundance of $\mathrm{P}$ - and Cl-bearing molecules relative to methanol detected towards the one-beam offset position from IRAS 162932422 B versus that measured in $67 \mathrm{P} / \mathrm{C}-\mathrm{G}$. Each molecule is marked with a unique colour. The shaded region corresponds to an order of magnitude scatter about the linear correlation. The Pearson $(r)$ and Spearman's $(\rho)$ correlation coefficients are given in the upper left-hand corner. 'up.lim.' indicates the values that are protostellar upper limits; and 'env.' indicates the value that is an estimate based on the circumbinary envelope.

disc around source B that have been derived for a region 0'.5 in size and stem from the series of papers from the PILS team at a relative accuracy of 10-20 percent. Variations in the $T_{\mathrm{ex}}$ of $\sim 20$ per cent $\left(25-60 \mathrm{~K}\right.$ for $\left.T_{\mathrm{ex}}=125-300 \mathrm{~K}\right)$ change the derived column densities by $<10$ percent (Jørgensen et al. 2018). Some previously unpublished and newly derived column densities (or at least upper limits) that are used for this study are presented in Appendix A. For molecules for which PILS data are insufficient, either estimates or ranges are provided based on earlier observations. All the protostellar and cometary abundances used for the analysis are tabulated in Tables 1 and 2, respectively. The error bars have been computed by considering [minimum, maximum] ratio ranges for the case of asymmetric errors, and via error propagation equations of normally distributed values for the case of symmetric errors. The full inventory of species detected towards IRAS 16293-2422 is provided in Appendix C.

Fig. 1 displays the abundance of $\mathrm{CHO}$-bearing molecules relative to methanol $\left(\mathrm{CH}_{3} \mathrm{OH}\right)$ detected towards the one-beam offset position from source $\mathrm{B}$ versus that measured in $67 \mathrm{P} / \mathrm{C}-\mathrm{G}$. The reference species selected is $\mathrm{CH}_{3} \mathrm{OH}$ for the $\mathrm{CHO}$-bearing family, because it is thought to form on grain surfaces via sequential hydrogenation of CO (Tielens \& Hagen 1982; Fuchs et al. 2009) and to be a key precursor to the synthesis of larger O-bearing complex organic molecules (Garrod, Weaver \& Herbst 2008; Fedoseev et al. 2017). The ROSINA mass spectrometer cannot unambiguously distinguish isomers as they have the same mass; hence, the measurements at mass $44 \mathrm{u} / \mathrm{e}$ are a combination of acetaldehyde $\left(\mathrm{CH}_{3} \mathrm{CHO}\right)$, ethylene oxide $\left(\mathrm{c}-\mathrm{C}_{2} \mathrm{H}_{4} \mathrm{O}\right)$, and vinyl alcohol $\left(\mathrm{CH}_{2} \mathrm{CHOH}\right)$; at mass $46 \mathrm{u} / \mathrm{e}$ are a combination of ethanol $\left(\mathrm{C}_{2} \mathrm{H}_{5} \mathrm{OH}\right)$ and dimethyl ether $\left(\mathrm{CH}_{3} \mathrm{OCH}_{3}\right)$; at mass $60 \mathrm{u} / \mathrm{e}$ are a combination of methyl formate $\left(\mathrm{HCOOCH}_{3}\right)$, glycolaldehyde $\left(\mathrm{CH}_{2} \mathrm{OHCHO}\right)$, and acetic acid $\left(\mathrm{CH}_{3} \mathrm{COOH}\right)$; and at mass $62 \mathrm{u} / \mathrm{e}$ are a combination of both ethylene glycol forms $\left(\left(\mathrm{CH}_{2} \mathrm{OH}\right)_{2}\right)$ and methoxymethanol $\left(\mathrm{CH}_{3} \mathrm{OCH}_{2} \mathrm{OH}\right)$. Spectroscopic observations have the ability to distinguish isomers; 
Table 1. Quantities of volatiles towards IRAS 16293-2422 B as observed with ALMA on protoplanetary disc-scales ${ }^{a}$.

\begin{tabular}{|c|c|c|c|c|}
\hline Species & Name & $\mathrm{N}\left(\mathrm{cm}^{-2}\right)^{b}$ and assumptions & Reference & $T_{\text {ex }}(\mathrm{K})$ \\
\hline $\mathrm{H}_{2} \mathrm{O}$ & Water & $\begin{array}{l}\text { source A est. } \\
3.3 \times 10^{21}\end{array}$ & $\begin{array}{l}\text { Persson, Jørgensen \& van Dishoeck }(2013)^{c} \\
-\end{array}$ & $124 \pm 12$ \\
\hline $\mathrm{O}_{2}$ & Molecular oxygen & $\begin{array}{l}\text { assuming tent. detection } \\
\quad<2.0 \times 10^{20}\end{array}$ & $\begin{array}{l}\text { Taquet et al. (2018) } \\
-\end{array}$ & $\begin{array}{c}- \\
300\end{array}$ \\
\hline $\mathrm{CO}$ & Carbon monoxide & $1.0 \times 10^{20}$ & this work (Appendix A) & $100-150$ \\
\hline $\mathrm{CH}_{3} \mathrm{OH}$ & Methanol & $1.0 \times 10^{19}$ & Jørgensen et al. $(2016,2018)^{d}$ & 300 \\
\hline $\mathrm{H}_{2} \mathrm{CO}$ & Formaldehyde & $1.9 \times 10^{18}$ & Persson et al. (2018) & $106 \pm 13$ \\
\hline $\mathrm{C}_{2} \mathrm{H}_{5} \mathrm{OH}$ & Ethanol & $2.3 \times 10^{17}$ & Jørgensen et al. (2018) & 300 \\
\hline $\mathrm{CH}_{3} \mathrm{OCH}_{3}$ & Dimethyl ether & $2.4 \times 10^{17}$ & Jørgensen et al. (2018) & 125 \\
\hline $\mathrm{HCOOCH}_{3}$ & Methyl formate & $2.6 \times 10^{17}$ & Jørgensen et al. (2018) & 300 \\
\hline $\mathrm{CH}_{2} \mathrm{OHCHO}$ & Glycolal dehyde & $3.2 \times 10^{16}$ & Jørgensen et al. (2016) ${ }^{d}$ & 300 \\
\hline $\mathrm{CH}_{3} \mathrm{COOH}$ & Acetic acid & $2.8 \times 10^{15}$ & Jørgensen et al. (2016) ${ }^{d}$ & 300 \\
\hline $\mathrm{CH}_{3} \mathrm{CHO}$ & Acetaldehyde & $1.2 \times 10^{17}$ & Jørgensen et al. (2018) & 125 \\
\hline $\mathrm{c}-\mathrm{C}_{2} \mathrm{H}_{4} \mathrm{O}$ & Ethylene oxide & $5.4 \times 10^{15}$ & Lykke et al. (2017) & 125 \\
\hline $\mathrm{CH}_{2} \mathrm{CHOH}$ & Vinyl alcohol & $<1.8 \times 10^{15}$ & Lykke et al. (2017) & 125 \\
\hline $\mathrm{HCOOH}$ & Formic acid & $5.6 \times 10^{16}$ & Jørgensen et al. (2018) & 300 \\
\hline aGg'-((CH$\left.\left.{ }_{2} \mathrm{OH}\right)_{2}\right)$ & aGg'-Ethylene glycol & $5.2 \times 10^{16}$ & Jørgensen et al. (2016) ${ }^{d}$ & 300 \\
\hline gGg'-((CH$\left.\left.{ }_{2} \mathrm{OH}\right)_{2}\right)$ & gGg'-Ethylene glycol & $4.7 \times 10^{16}$ & Jørgensen et al. (2016) ${ }^{d}$ & 300 \\
\hline $\mathrm{CH}_{3} \mathrm{OCH}_{2} \mathrm{OH}$ & Methoxymethanol & $1.4 \times 10^{17}$ & Manigand et al. submitted & 130 \\
\hline $\mathrm{C}_{2} \mathrm{H}_{5} \mathrm{CHO}$ & Propanal & $2.2 \times 10^{15}$ & Lykke et al. (2017) & 125 \\
\hline$\left(\mathrm{CH}_{3}\right)_{2} \mathrm{CO}$ & Acetone & $1.7 \times 10^{16}$ & Lykke et al. (2017) & 125 \\
\hline $\mathrm{NH}_{2} \mathrm{CHO}$ & Formamide & $9.5 \times 10^{15}$ & Coutens et al. (2016) & 300 \\
\hline $\mathrm{NH}_{3}$ & Ammonia & $\begin{array}{l}\text { circumbinary envelope est. } \\
\qquad 6.1 \times 10^{19}\end{array}$ & $\begin{array}{l}\text { Hily-Blant et al. (2010), } \\
\text { van Dishoeck et al. (1995), } \\
\text { Mundy et al. (1990), } \\
\text { Mizuno et al. (1990) }\end{array}$ & $\begin{array}{c}8-30 \\
- \\
-\end{array}$ \\
\hline $\mathrm{HCN}$ & Hydrogen cyanide & $5.0 \times 10^{16}$ & this work (Appendix A) & 120 \\
\hline $\mathrm{HNC}$ & Hydrogen isocyanide & $<5.0 \times 10^{16}$ & this work (Appendix A) & 120 \\
\hline $\mathrm{CH}_{3} \mathrm{CN}$ & Methyl cyanide & $4.0 \times 10^{16}$ & Calcutt et al. (2018b) & $110 \pm 10$ \\
\hline $\mathrm{CH}_{3} \mathrm{NC}$ & Methyl isocyanide & $2.0 \times 10^{14}$ & Calcutt et al. (2018a) & $150 \pm 20$ \\
\hline HNCO & Isocyanic acid & $3.7 \times 10^{16}$ & Ligterink et al. (2017) & 100 \\
\hline $\mathrm{HOCN}$ & Cyanic acid & $<3.0 \times 10^{13}$ & Ligterink et al. (2017) & 100 \\
\hline $\mathrm{HC}_{3} \mathrm{~N}$ & Cyanoacetylene & $1.8 \times 10^{14}$ & Calcutt et al. (2018b) & $100 \pm 20$ \\
\hline $\mathrm{H}_{2} \mathrm{~S}$ & Hydrogen sulphide & $1.7 \times 10^{17}$ & Drozdovskaya et al. (2018) & 125 \\
\hline OCS & Carbonyl suplhide & $2.5 \times 10^{17}$ & Drozdovskaya et al. (2018) & 125 \\
\hline $\mathrm{CH}_{3} \mathrm{SH}$ & Methyl mercaptan & $4.8 \times 10^{15}$ & Drozdovskaya et al. (2018) & 125 \\
\hline $\mathrm{CS}$ & Carbon monosulphide & $3.9 \times 10^{15}$ & Drozdovskaya et al. (2018) & 125 \\
\hline $\mathrm{H}_{2} \mathrm{CS}$ & Thioformaldehyde & $1.3 \times 10^{15}$ & Drozdovskaya et al. (2018) & 125 \\
\hline $\mathrm{S}_{2}$ & Disulphur & $<1.9 \times 10^{16}$ & Drozdovskaya et al. (2018) & 125 \\
\hline $\mathrm{SO}_{2}$ & Sulphur dioxide & $1.3 \times 10^{15}$ & Drozdovskaya et al. (2018) & 125 \\
\hline SO & Sulphur monoxide & $4.4 \times 10^{14}$ & Drozdovskaya et al. (2018) & 125 \\
\hline $\mathrm{C}_{2} \mathrm{H}_{5} \mathrm{SH}$ & Ethyl mercaptan & $<3.2 \times 10^{15}$ & Drozdovskaya et al. (2018) & 125 \\
\hline $\mathrm{H}_{2} \mathrm{~S}_{2}$ & Disulphane & $<7.9 \times 10^{14}$ & Drozdovskaya et al. (2018) & 125 \\
\hline $\mathrm{HS}_{2}$ & Disulphanide & $<4.4 \times 10^{14}$ & Drozdovskaya et al. (2018) & 125 \\
\hline $\mathrm{PO}$ & Phosphorus monoxide & $<4.4 \times 10^{14}$ & this work (Appendix A) & 125 \\
\hline $\mathrm{PN}$ & Phosphorus mononitride & $<2.1 \times 10^{13}$ & this work (Appendix A) & 125 \\
\hline $\mathrm{HCl}$ & Hydrogen chloride & $\begin{array}{l}\text { circumbinary envelope est. } \\
\qquad<3.4 \times 10^{16}\end{array}$ & Peng et al. $(2010)^{f}$ & $\begin{array}{c}- \\
80\end{array}$ \\
\hline $\mathrm{CH}_{3} \mathrm{Cl}$ & Methyl chloride & $4.6 \times 10^{14}$ & Fayolle et al. (2017) & $102 \pm 3$ \\
\hline $\mathrm{NH}_{2} \mathrm{CH}_{2} \mathrm{COOH}$ & Glycine & $<9.2 \times 10^{14}$ & this work (Appendix A) & 300 \\
\hline
\end{tabular}

Notes: ${ }^{a}$ The assumed source size is 0.5 ; and $\eta_{\mathrm{BF}}=$ sourcesize ${ }^{2} /\left(\right.$ sourcesize $^{2}+$ beamsize $\left.^{2}\right)$, when the source size is smaller than the beam size; and $\eta_{\mathrm{BF}}=$ sourcesize ${ }^{2} / \mathrm{beamsize}^{2}$, when the beam size is smaller than the source size.

${ }^{b}$ A correction factor for the coupling of line emission with the emission from dense dust at $T_{\mathrm{bg}}$ needs to be accounted for when deriving the column density at the one-beam offset position from source B. (Persson et al. 2018) used a consistently derived value of 1.1658. Ligterink et al. (2017); Fayolle et al. (2017) and Manigand et al. (submitted) also consistently corrected for $T_{\mathrm{bg}}=21 \mathrm{~K}$. Jørgensen et al. (2016, 2018); Calcutt et al. (2018b,a) applied correction factors of 1.14 and 1.05 for $T_{\mathrm{ex}}=125$ and $300 \mathrm{~K}$, respectively (corresponding to $T_{\text {bg }}=21 \mathrm{~K}$ ). Coutens et al. (2016); Lykke et al. (2017); Drozdovskaya et al. (2018); Taquet et al. (2018) did not account for this factor in the published column densities, and hence, these values have been corrected in this work through division by 1.14 or 1.05 depending on the $T_{\text {ex }}$ of the molecule in question. This has either been explicitly stated by the authors or has been conveyed via private communication. For the column densities of PO, PN, and glycine that are newly derived in this work, these correction factors have been applied. For the column densities of $\mathrm{CO}, \mathrm{HCN}$, and HNC that are newly derived in this work, these correction factors are not applied due to highly uncertain spatial distributions of these molecules. The accuracy of the derived column densities is $10-20$ per cent. Variations in $T_{\mathrm{ex}}$ of $\sim 20$ per cent $\left(25-60 \mathrm{~K}\right.$ for $\left.T_{\mathrm{ex}}=125-300 \mathrm{~K}\right)$ change the derived column densities by $<10$ per cent (Jørgensen et al. 2018).

${ }^{c}$ For a beamsize $=0^{\prime \prime} \cdot 2, N\left(\mathrm{H}_{2}^{18} \mathrm{O}\right)=9.5 \times 10^{17} \mathrm{~cm}^{-2}$. Assuming ${ }^{16} \mathrm{O} /{ }^{18} \mathrm{O}=557$ and using $\eta_{\mathrm{BF}} \approx 6$, gives $N\left(\mathrm{H}_{2} \mathrm{O}\right)=N\left(\mathrm{H}_{2}^{18} \mathrm{O}\right) \times \eta_{\mathrm{BF}} \times\left({ }^{16} \mathrm{O} /{ }^{18} \mathrm{O}\right)$ as the value used in this work for a source size of 0 !' 5 under the assumption of homologous emission.

${ }^{d}$ The published column densities have been reduced by a factor of 2.136 to account for the shift from the half-beam offset position to the full-beam offset position from source B. ${ }^{e}$ Several estimates exist in the literature, but all are only for the circumbinary envelope. Hily-Blant et al. $(2010): T_{\text {ex }}=8-10 \mathrm{~K}$, beamsize $=12-18$ arcsec, $N\left(\mathrm{NH}_{3}\right)=$ $20-3.5 \times 10^{15} \mathrm{~cm}^{-2}$. van Dishoeck et al. (1995): $T_{\mathrm{ex}}=25 \pm 5 \mathrm{~K}$, beamsize $=20 \operatorname{arcsec}, N\left(\mathrm{NH}_{2} \mathrm{D}\right)=1.9 \times 10^{14} \mathrm{~cm}^{-2} ;$ assuming $\mathrm{D} / \mathrm{H}=0.05-0.005$ gives $N(\mathrm{NH} 3)=$ $N\left(\mathrm{NH}_{2} \mathrm{D}\right) \times(\mathrm{H} / \mathrm{D})=3.8-38 \times 10^{15} \mathrm{~cm}^{-2}$. Mundy, Wootten \& Wilking (1990): $T_{\mathrm{ex}}=15-20 \mathrm{~K}$, beamsize $=20 \operatorname{arcsec}, N\left(\mathrm{NH}_{3}\right)=2 \times 10^{15} \mathrm{~cm}^{-2} . \mathrm{Mizuno}$ et al. $(1990): T_{\mathrm{ex}}=$ $15 \mathrm{~K}$, beamsize $=40 \operatorname{arcsec}, N\left(\mathrm{NH}_{3}\right)=8 \times 10^{14} \mathrm{~cm}^{-2}$; assuming sourcesize $=20 \operatorname{arcsec}$ yields $\eta_{\mathrm{BF}}=0.2$ and gives $N\left(\mathrm{NH}_{3}\right) / \eta_{\mathrm{BF}}=4 \times 10^{15} \mathrm{~cm}{ }^{-2}$. Taking all these estimates for a beamsize $=20$ arcsec yields a range: $N\left(\mathrm{NH}_{3}\right)=2.0 \times 10^{15}-3.8 \times 10^{16} \mathrm{~cm}^{-2}$. Now assuming sourcesize $=0$ '. 5 yields $\eta_{\mathrm{BF}} \approx 6 \times 10^{-4}$ and results in the $N(\mathrm{NH}) / \eta_{\mathrm{BF}}$ value used in this work.

${ }^{f}$ Only one estimate exists in the literature, for the circumbinary envelope scales from Peng et al. $(2010)$ for beamsize $=13^{\prime \prime} .5, N(\mathrm{HCl})=4.7 \times 10^{13} \mathrm{~cm}^{-2}$; assuming sourcesize $=$ 0.5 yields $\eta_{\mathrm{BF}}=1 / 730$ and results in the $N(\mathrm{HCl}) / \eta_{\mathrm{BF}}$ value used in this work. 
Table 2. Bulk quantities of volatiles in $67 \mathrm{P} / \mathrm{C}-\mathrm{G}$ as measured with ROSINA ${ }^{a}$.

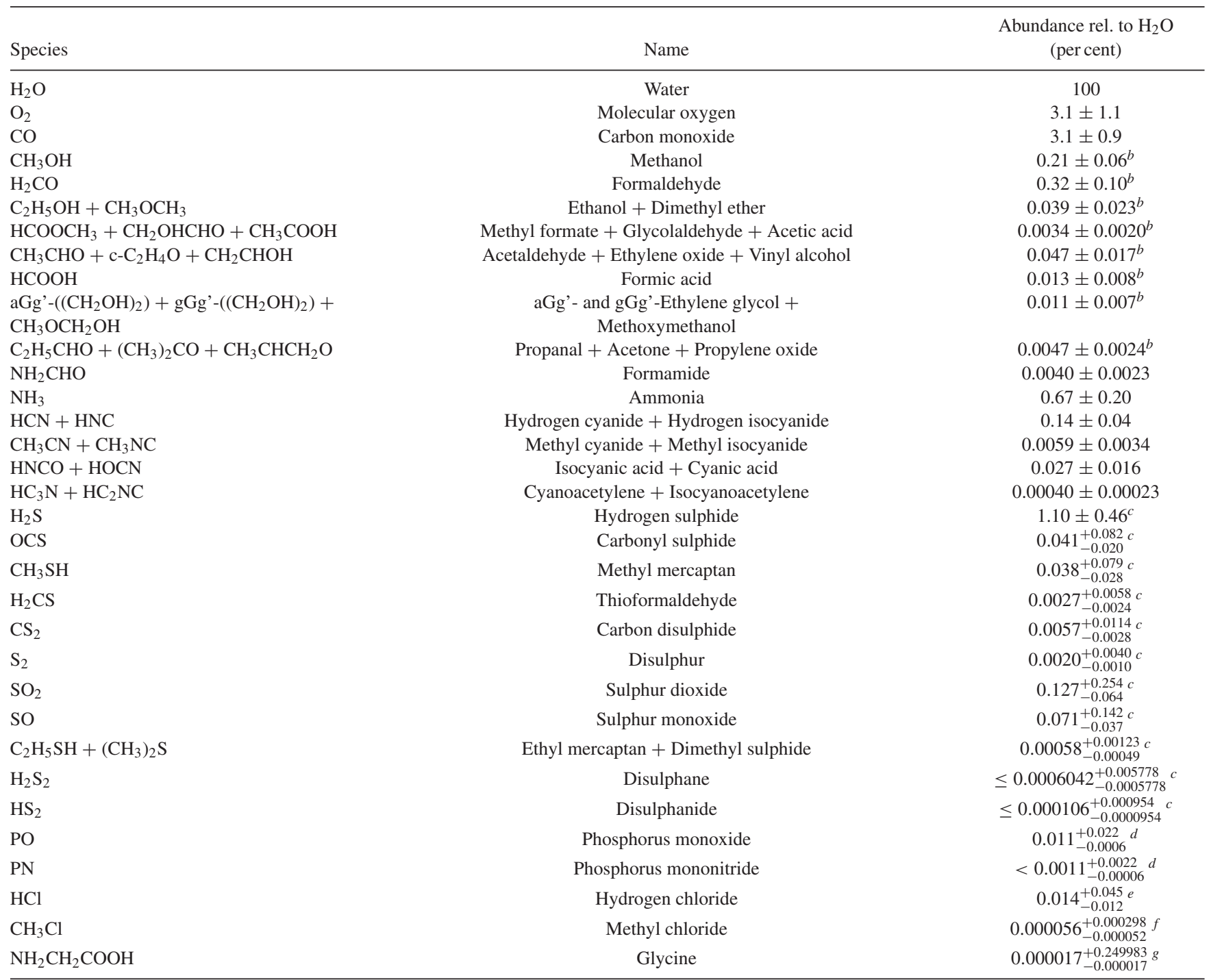

Notes: ${ }^{a}$ All bulk abundances are from Rubin et al. 2019, unless indicated otherwise (see also Altwegg et al. 2019).

${ }^{b}$ Schuhmann et al. 2019.

${ }^{c}$ Calmonte et al. (2016)

${ }^{d}$ Rivilla et al. in preparation.

${ }^{e}$ Dhooghe et al. (2017)

${ }^{f}$ Fayolle et al. (2017)

${ }^{g}$ Hadraoui et al. (in press) with error bars based on the range of glycine abundances reported in Altwegg et al. (2016).

however, the observed protostellar abundances have been summed to make an appropriate comparison with cometary measurements. The cometary value for glycine does not stem from the same period as bulk abundances for the majority of the other species analysed in this work, as continuous data were not available for this molecule during the mission. The value used stems from dedicated models of glycine in $67 \mathrm{P} / \mathrm{C}-\mathrm{G}$, which inferred it to be desorbing from the nucleus and from icy mantles of dust particles ejected from the nucleus into the coma (i.e. a distributed source, Altwegg et al. 2016), while being mixed with water ice in both of these sources (Hadraoui et al. in press). The error bars are based on the range of measured glycine abundances reported in Altwegg et al. (2016). The protostellar abundances of $\mathrm{O}_{2}, \mathrm{CH}_{2} \mathrm{CHOH}$, and glycine are upper limits, which means that these points may shift lower along the ordinate. The protostellar abundance of $\mathrm{H}_{2} \mathrm{O}$ is an estimate based on the value derived for source A (see Table 1 for details). The $\mathrm{C}_{2} \mathrm{H}_{5} \mathrm{CHO}$ data point is a currently best-possible estimate, as the ROSINA measurement at mass $58 \mathrm{u} / \mathrm{e}$ is a combination of propanal $\left(\mathrm{C}_{2} \mathrm{H}_{5} \mathrm{CHO}\right)$, acetone $\left(\left(\mathrm{CH}_{3}\right)_{2} \mathrm{CO}\right)$, and propylene oxide $\left(\mathrm{CH}_{3} \mathrm{CHCH}_{2} \mathrm{O}\right)$, while the column density of the latter is currently unavailable for IRAS 16293-2422. The figure appears to display a linear correlation between the two sets of abundances (including those that are upper limits) with a Pearson correlation coefficient of 1.00 and a Spearman's correlation coefficient of 0.88 (at a two-tailed significance of $8.1 \times 10^{-5}$; Table 3 ), which implies that cometary and protostellar $\mathrm{CHO}$-bearing volatiles are related.

Fig. 2 displays the abundance of $\mathrm{N}$-bearing molecules relative to methyl cyanide $\left(\mathrm{CH}_{3} \mathrm{CN}\right)$ detected towards the one-beam offset position from source $\mathrm{B}$ versus that measured in $67 \mathrm{P} / \mathrm{C}-\mathrm{G}$. The ROSINA mass spectrometer measurement at mass $27 \mathrm{u} / \mathrm{e}$ is a 
Table 3. Correlation coefficients between volatiles towards IRAS 16293-2422 B on disc-scales and in the bulk of 67P/C-G.

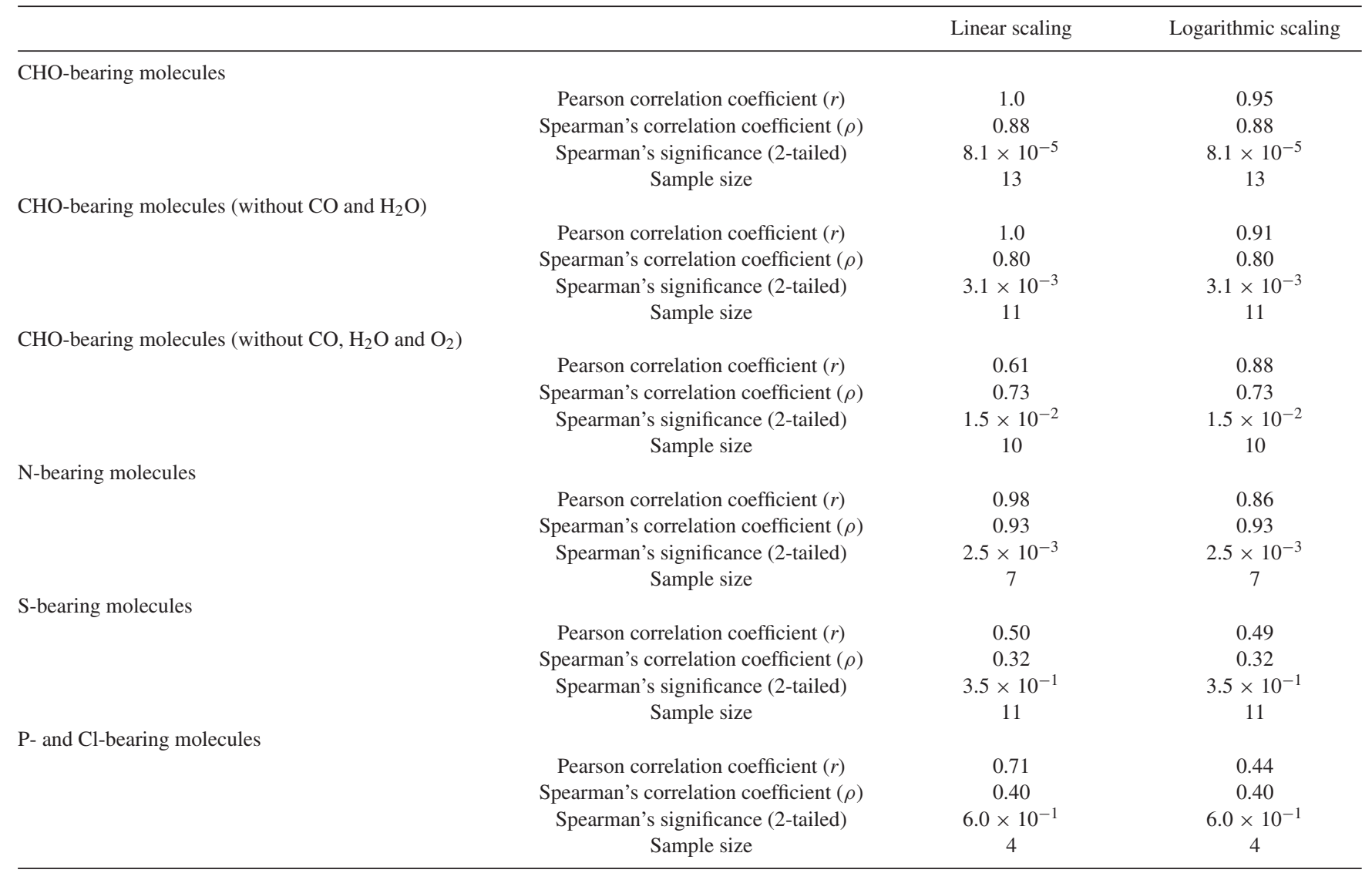

combination of hydrogen isocyanide (HNC) and hydrogen cyanide $(\mathrm{HCN})$; at mass $41 \mathrm{u} / \mathrm{e}$ is a combination of methyl isocyanide $\left(\mathrm{CH}_{3} \mathrm{NC}\right)$ and methyl cyanide; at mass $43 \mathrm{u} / \mathrm{e}$ is a combination of isocyanic acid (HNCO) and cyanic acid (HOCN); the protostellar values have been summed for these pairs of molecules accordingly. The cometary value for glycine is the best available, as discussed in the above paragraph. The protostellar abundances of HNC, HOCN, and glycine are upper limits, which means that these points may shift lower along the ordinate. The $\mathrm{HC}_{3} \mathrm{~N}$ data point is a currently best-possible estimate, as the ROSINA measurement at mass $51 \mathrm{u} / \mathrm{e}$ is a combination of cyanoacetylene $\left(\mathrm{HC}_{3} \mathrm{~N}\right)$ and isocyanoacetylene $\left(\mathrm{HC}_{2} \mathrm{NC}\right)$, while the column density of the latter is currently unavailable for IRAS 16293-2422. The protostellar abundance of $\mathrm{NH}_{3}$ is an estimated upper limit based on circumbinary envelopescale observations (see Table 1 for details). This figure appears to display a linear correlation between the two sets of abundances (including those that are upper limits) with a Pearson correlation coefficient of 0.98 and a Spearman's correlation coefficient of 0.93 (at a two-tailed significance of $2.5 \times 10^{-3}$; Table 3 ), which implies that cometary and protostellar N-bearing volatiles are related. The reference species selected is $\mathrm{CH}_{3} \mathrm{CN}$ for the $\mathrm{N}$-bearing family, whose origin is still unclear. It can be formed on grain surfaces with some contributions from gas-phase reactions (Calcutt et al. 2018b). $\mathrm{CH}_{3} \mathrm{CN}$ is the analogous 6-atom molecule with a methyl $\left(\mathrm{CH}_{3}-\right)$ functional group to $\mathrm{CH}_{3} \mathrm{OH}$. $\mathrm{CH}_{3} \mathrm{NC}$ had to be added to the chosen reference species $\mathrm{CH}_{3} \mathrm{CN}$ due to their identical mass being indistinguishable for the ROSINA instrument; however, the protostellar column density of $\mathrm{CH}_{3} \mathrm{NC}$ is two orders of magnitude lower than that of $\mathrm{CH}_{3} \mathrm{CN}$, and hence, likely only makes a minor difference.

Fig. 3 displays the abundance of S-bearing molecules relative to methyl mercaptan $\left(\mathrm{CH}_{3} \mathrm{SH}\right)$ detected towards the one-beam offset position from source B versus that measured in $67 \mathrm{P} / \mathrm{C}-\mathrm{G}$. In ROSINA data, the CS peak suffers from interference with the strong signal from abundant $\mathrm{CO}_{2}$ at mass $44 \mathrm{u} / \mathrm{e}$. Over a short flyby in 2015 March, when $\mathrm{CO}_{2}$ was scarcer, it was derived that all CS detected by ROSINA can be explained by the fragmentation of primarily $\mathrm{CS}_{2}$ and secondarily, of OCS (Calmonte et al. 2016). Therefore, the amount of CS as a radical in cometary ice is small, if existent at all. Summing the abundances of $\mathrm{CS}_{2}$ and OCS ices yields an upper limit for the amount of CS radicals incorporated into $67 \mathrm{P} / \mathrm{C}-\mathrm{G}$ at some point since the prestellar phase, assuming that all CS radicals are converted into either OCS or $\mathrm{CS}_{2}$ by the time they become constituents of cometary ice. This is the CS upper limit used in Fig. 3. Cometary values for $\mathrm{H}_{2} \mathrm{CS}, \mathrm{CH}_{3} \mathrm{SH}$, and $\mathrm{C}_{2} \mathrm{H}_{5} \mathrm{SH}$ do not stem from the same period as bulk abundances for the majority of the other species analysed in this work, as continuous data were not available for these two molecules during the mission. Hence, these two numbers may be somewhat less representative of the bulk, but are the best available (see Calmonte et al. 2016 for details). The cometary abundances of $\mathrm{HS}_{2}$ and $\mathrm{H}_{2} \mathrm{~S}_{2}$ are upper limits, which means that these points may shift to the left along the abscissa. The protostellar abundances of $\mathrm{S}_{2}, \mathrm{HS}_{2}$, $\mathrm{H}_{2} \mathrm{~S}_{2}$, and $\mathrm{C}_{2} \mathrm{H}_{5} \mathrm{SH}$ are upper limits, which means that these points may shift lower along the ordinate. The $\mathrm{C}_{2} \mathrm{H}_{5} \mathrm{SH}$ data point is a currently best-possible estimate, as the ROSINA measurement at 
this mass of $62 \mathrm{u} / \mathrm{e}$ is a combination of ethyl mercaptan $\left(\mathrm{C}_{2} \mathrm{H}_{5} \mathrm{SH}\right)$ and dimethyl sulphide $\left(\left(\mathrm{CH}_{3}\right)_{2} \mathrm{~S}\right)$, while spectroscopy of dimethyl sulphide is not yet available, which inhibits its search in the ALMA data. The figure appears to display a linear correlation between the two sets of abundances (including those that are upper limits) with a Pearson correlation coefficient of 0.50 and a Spearman's correlation coefficient of 0.32 (at a two-tailed significance of 0.35 ; Table 3), which implies that cometary and protostellar S-bearing volatiles are related. These $r$ - and $\rho$-values are lower than that of $\mathrm{CHO}-$ and N-bearing species, potentially due to the larger fraction of upper limits and best-effort estimates used in the S-bearing family. Furthermore, S-bearing molecules span a smaller range of relative abundances than the $\mathrm{CHO}$ - and $\mathrm{N}$-bearing species. The reference species selected is $\mathrm{CH}_{3} \mathrm{SH}$ for the $\mathrm{S}$-bearing family, which is formed on grain surfaces from atomic sulphur and CS hydrogenations (Vidal et al. 2017; Lamberts 2018). $\mathrm{CH}_{3} \mathrm{SH}$ is the analogous 6atom molecule with a methyl $\left(\mathrm{CH}_{3}-\right)$ functional group to $\mathrm{CH}_{3} \mathrm{OH}$ and $\mathrm{CH}_{3} \mathrm{CN}$.

Fig. 4 displays the abundance of P- and Cl-bearing molecules relative to methanol detected towards the one-beam offset position from source B versus that measured in $67 \mathrm{P} / \mathrm{C}-\mathrm{G}$. The cometary abundance of $\mathrm{PN}$ is an upper limit, as its mass peak overlaps with those of CHS and ${ }^{13} \mathrm{CS}$, and suffers from strong interference with the peak of ${ }^{13} \mathrm{CO}_{2}$ (Rivilla et al. in preparation). The cometary values for $\mathrm{PN}, \mathrm{PO}$, and $\mathrm{CH}_{3} \mathrm{Cl}$ do not stem from the same period as bulk abundances for the majority of the other species analysed in this work, as continuous data were not available for these molecules during the mission. Hence, as for $\mathrm{H}_{2} \mathrm{CS}, \mathrm{CH}_{3} \mathrm{SH}$, and $\mathrm{C}_{2} \mathrm{H}_{5} \mathrm{SH}$, these numbers may be somewhat less representative of the bulk, but are the best available (see Rivilla et al. in preparation and Fayolle et al. 2017 for details). The protostellar abundances for $\mathrm{PO}$ and PN are the currently best-available upper limit estimates, which will soon be tested with new dedicated ALMA observations (project-id: 2018.1.01496, PI: Víctor M. Rivilla). The protostellar abundance of $\mathrm{HCl}$ is an estimate based on circumbinary envelopescale observations (see Table 1 for details). The figure appears to display a linear correlation between the two sets of abundances (including those that are upper limits) with a Pearson correlation coefficient of 0.71 and a Spearman's correlation coefficient of 0.40 (at a two-tailed significance of 0.6; Table 3), although more data points are desirable. The linear correlation is not one-to-one, but is offset, which may be a result of $\mathrm{CH}_{3} \mathrm{OH}$ being chosen as the reference species. It is not clear what the best reference species is for these exotic species. The correlation tentatively suggests that cometary and protostellar P- and Cl-bearing volatiles are related. These chemical families have never been probed before.

The correlations in Figs 1-4 vary in strength and significance. The relative abundances investigated span a wide range; hence, the correlation coefficients have also been computed with logarithmic scaling (the coefficients given in the figures are derived with the plotted linear scaling). Table 3 summarizes all the correlation coefficients and their significance. It can be seen that logarithmic scaling lowers the strength of the correlations somewhat in terms of the Pearson correlation coefficients; however, they remain statistically significant. For the case of CHO-bearing molecules, that span the largest range of relative abundances, the correlation has also been scrutinized upon exclusion of simpler species, specifically $\mathrm{H}_{2} \mathrm{O}$, $\mathrm{CO}$, and $\mathrm{O}_{2}$. This results in a significant reduction of the Pearson and Spearman's correlation coefficients to 0.61 and 0.73 (at a twotailed significance of 0.015 ), respectively. This supports there being significantly more complex organic $\mathrm{CHO}$-bearing species in the comet than towards the protostar.
Beyond the chemical relevance of the three reference species $\left(\mathrm{CH}_{3} \mathrm{OH}, \mathrm{CH}_{3} \mathrm{CN}\right.$, and $\left.\mathrm{CH}_{3} \mathrm{SH}\right)$ for their respective chemical families, they are also expected to be present predominantly on small scales in the hot inner regions around protostars due to their high desorption energies (5534, 4680, and $4000 \mathrm{~K}$, respectively). This consequently makes these molecules more relevant for tracing discmaterials, rather than those that may be thermally desorbed already at lower temperatures, which are easily attained on larger envelopescales in the system (as is the case for $\mathrm{HCN}$, for example). The reference species $\mathrm{CH}_{3} \mathrm{OH}, \mathrm{CH}_{3} \mathrm{CN}$, and $\mathrm{CH}_{3} \mathrm{SH}$ ensure one-to-one correlations in Figs 1-3 for the CHO-, N-, and S-bearing families, respectively. A choice of a common reference species (such as $\mathrm{CH}_{3} \mathrm{OH}$ or $\mathrm{H}_{2} \mathrm{O}$ ) for all molecules preserves the linear correlations for the members of a single chemical family, but introduces a scaling factor to the linear correlation, i.e. it is no longer one-to-one. This can be seen by comparing Fig. B1 with Figs B2 and B3. This is analogous to the offset seen for $\mathrm{P}$-and $\mathrm{Cl}$-bearing molecules when using methanol as the reference species (Fig. 4).

\subsection{Caveats}

\subsubsection{Missed reservoirs}

Not all major reservoirs have been probed with the observations discussed in this work. As discussed in the previous section, it is not possible to probe individual isomers unambiguously with ROSINA measurements for $67 \mathrm{P} / \mathrm{C}-\mathrm{G}$, as well as the interfering $\mathrm{CS}$ and ${ }^{13} \mathrm{CO}_{2}$. Meanwhile, with ALMA data at radio frequencies that are sensitive to rotational lines of molecules, it is not possible to determine the abundance of symmetric molecules such as $\mathrm{N}_{2}, \mathrm{CH}_{4}, \mathrm{CS}_{2}$, and $\mathrm{CO}_{2}$; and of atoms such as S. Only non-trivial combinations of data from different instruments can tackle these missed reservoirs of volatiles.

\subsubsection{Representability of the targets}

There is no evidence to suggest that $67 \mathrm{P} / \mathrm{C}-\mathrm{G}$ is in any way an atypical comet. Its bilobate shape is similar to that of comet 103P/Hartley 2 (A'Hearn 2011) among others, and the transNeptunian object (486958) $2014 \mathrm{MU}_{69}$ (Stern et al. 2019). The topographically heterogeneous surface of $67 \mathrm{P} / \mathrm{C}-\mathrm{G}$ dominated by smooth-floored pits appears to be most similar to 81P/Wild 2 (Birch et al. 2017). Its dominant volatile has been shown to be water ice that is hidden in the interior and almost completely absent from the surface, which is again typical of all the comets studied thus far (Filacchione et al. 2016a). The low-density and high porosity (72-74 per cent) of the nucleus of $67 \mathrm{P} / \mathrm{C}-\mathrm{G}$ is comparable to that of comet 9P/Tempel 1 (Pätzold et al. 2016). The coma has been quite tenuous in comparison to the brightest comets that have been observed, such as Hale-Bopp whose production rates were roughly two orders of magnitude higher (Altwegg et al. 2019). The chemical richness observed on $67 \mathrm{P} / \mathrm{C}-\mathrm{G}$ is very likely a mere consequence of the superior measurement techniques (long-term monitoring at close distances coupled with high sensitivity of Rosetta's scientific payload). This is supported by the detection of ethylene glycol and formamide on comets C/2012 F6 (Lemmon) and C/2013 R1 (Lovejoy), as well as ethanol and glycolaldehyde in the latter target (Biver et al. 2014, 2015). The determined volatile composition does not show any major differences from that seen in other comets (as reviewed in Cochran et al. 2015; Dello Russo et al. 2016).

Likewise, there is no firm support for IRAS 16293-2422 being in any way a unique young stellar object in terms of the chemical abundances and diversity that is observed (Jørgensen, Schöier \& 
van Dishoeck 2004; Taquet et al. 2015). The short distance to this source facilitates the detection of all the minor and weakly emitting molecules. For example, complex organic molecule abundances towards L483 as observed with ALMA compare well to those of IRAS 16293-2422 B (Jacobsen et al. 2019). From the point of view of the physical structure, there also does not seem to be anything out of the ordinary within the large morphological diversity that is seen in star-forming regions. Multiplicity appears to be common for Class 0 and I sources (Tobin et al. 2016). Source A has also been suggested to be binary in itself (e.g. Hernández-Gómez et al. 2019a). The deuteration of water as measured via the $\mathrm{HDO} / \mathrm{H}_{2} \mathrm{O}$ ratio in IRAS 16293-2422 is in range of other deeply embedded low-mass sources on the same spatial scales (fig. 6 of Persson et al. 2014), hence, suggesting no drastic temperature differences at the time of water molecule formation in such systems. Further work remains to be done for a larger sample of isolated protostars and the more classical hot corino sources.

On the other hand, it is thought that binaries that are separated by more than disc-scales will not be significantly impacted by neither the passive (heating of the inner collapsing envelope by the protostellar luminosity) nor active (heating by shocks) heating nor the UV flux of their companion. Such conclusions were reached based on ${ }^{13} \mathrm{CO}$ observations across samples of low-mass protostars (van Kempen et al. 2009; Y1ldiz et al. 2013, 2015). The only parts of low-mass binary systems that will be heated and UV-irradiated on scales of up to $\sim 1000$ au are the outflow cavities and the cavity walls. This result even holds for species that are enhanced in abundance by UV (e.g. c- $\mathrm{C}_{3} \mathrm{H}_{2}$; Murillo et al. 2018a). In IRAS 16293-2422, source $\mathrm{B}$ appears to lie at a projected position that overlaps with the north-west outflow stemming from source A (Kristensen et al. 2013; Girart et al. 2014; van der Wiel et al. 2019). Unfortunately, the inclination angle of the north-west/southeast outflows of source A with respect to the plane of the sky and with respect to source B or the 'bridge' remains unknown. However, the emission line profiles near source B do not show any evidence for shocks or outflows impinging on to source B from the outside. Consequently, source A is thought to not affect neither the temperature structure nor the UV field in the vicinity of source B, in contrast to a source such as B1-bW (Hirano \& Liu 2014). This implies that the binary nature of IRAS 16293-2422 is likely not significant in the context of analyses on disc-scales carried out in this work.

\section{DISCUSSION}

\subsection{Chemical links between comets and the ISM}

The ALMA data analysed in Section 3 are sensitive to the gases present $\sim 70$ au away from protostar B. It is anticipated that the observed gases represent the volatiles that are being transported into the forming protoplanetary disc, which have recently been thermally desorbed upon approach to the protostar, and are likely still present in the solid phase in colder regions of the disc. Thus, these ALMA data provide a unique view into the chemical composition of planetand comet-forming materials in the low-mass source IRAS 16293 $2422 \mathrm{~B}$. The focal one $0.5 \mathrm{arcsec}$ beam offset position of this work represents some of the most accurate and systematically derived relative abundances for volatiles in a forming Solar-like disc due to the use of optically thin isotopologues, no beam dilution, and a relative accuracy of 10-20 per cent on the derived column densities.

The ROSINA data used in Section 3 pertain to in situ measurements of the coma gases of $67 \mathrm{P} / \mathrm{C}-\mathrm{G}$ with the majority of the uncertainties being $\sim 30$ percent. These measurements are unique due to the continuous monitoring carried out by the Rosetta mission, which allows bulk abundances to be derived for the first time rather than mere snap shots at an isolated moment in time. Hence, the cometary values analysed in this study are the most representative available for the building blocks of our Solar System.

Figs 1-4 display correlations between CHO-, N-, S-, P-, and Cl-bearing volatiles observed in the protostar IRAS 16293-2422 B and comet $67 \mathrm{P} / \mathrm{C}-\mathrm{G}$ with Pearson correlation coefficients in the $[0.50,1.0]$ and Spearman's correlation coefficients in the $[0.32$, 0.93 ] ranges. These correlations suggest that volatiles in all lowmass Solar-like systems may be comparable and that some degree of preservation occurs for volatiles from the protostellar phases into comets. This implies that the composition of planetesimals is set, to some extent, in the youngest embedded phase of star formation. Scatter, up to an order of magnitude, is observed. This is particularly noticeable when exploring smaller ranges of relative abundances, such as the case for $\mathrm{S}$-bearing molecules and $\mathrm{CHO}$-bearing complex organic molecules (i.e. excluding $\mathrm{H}_{2} \mathrm{O}, \mathrm{CO}$, and $\mathrm{O}_{2}$ in Fig. 1). This may stem from the inclusion of upper limits in the analysis, or may be a natural consequence of the slightly different physical evolution of our Solar System from that of IRAS 16293-2422 B.

Figs 1 and 2 show that the relative abundance ratios of $67 \mathrm{P} / \mathrm{C}-\mathrm{G}$ tend to be higher than those of IRAS 16293-2422 for CHO- and $\mathrm{N}$-bearing species. This may indicate that relative to the reference species of a molecular family, the molecules considered in this work have been destroyed at the position near IRAS 16293-2422 B investigated with ALMA data in this work. Potential destruction may occur through gas-phase chemistry upon thermal desorption. Alternatively, it may be that more of the investigated molecules have been produced by the time of incorporation into the comet. For example, chemical modelling suggests that $\mathrm{CO}$ will be converted to $\mathrm{CH}_{3} \mathrm{OH}, \mathrm{CO}_{2}$, and hydrocarbons within protoplanetary discs (Bosman, Walsh \& van Dishoeck 2018). Higher relative quantities may stem from older material, which has given chemical reactions more time to produce more chemically complex species at elevated dust temperatures and UV fluxes during collapse through grainsurface chemical reactions (e.g. Drozdovskaya et al. 2014, 2016). Finally, earlier works have indicated that the amount of methanol in comets is generally lower than in protostellar regions (Öberg et al. 2011). This could also result in higher relative abundances being seen for $67 \mathrm{P} / \mathrm{C}-\mathrm{G}$ when using $\mathrm{CH}_{3} \mathrm{OH}$ as a reference species (Figs 1 and B2). Only a dedicated model combining cometesimal formation, global physical evolution of the star-disc system, and simultaneous chemistry can shed light on these scenarios.

In comparison to earlier work, the strong correlation between Hale-Bopp's and ISM relative abundances of CHO- and N-bearing molecules (figs $3 \mathrm{a}$ and $\mathrm{b}$ of Bockelée-Morvan et al. 2000) have now been confirmed for the case of a Jupiter-family comet, 67P/C-G, and for disc-scale rather than cloud-scale materials. Such correlations for the case of S-bearing species have been established for the first time in this work. This connection may have been missed due to the data on Hale-Bopp (fig. 3c of Bockelée-Morvan et al. 2000) being a mere snapshot of its coma composition at the time that the observations were carried out. S-bearing species, especially $\mathrm{S}_{3}$ and $\mathrm{S}_{4}$, are strongly associated with high dust densities in the coma (Calmonte et al. 2016), potentially implying that remote observations may be picking up S-bearing species originating from the nucleus as well as from a distributed source (Cottin \& Fray 2008; Altwegg et al. 2017b). Contributions from S-bearing species stemming from the refractory dust may mask the correlation in volatiles. Alternatively, the abundances of S-bearing molecules may be more variable than others across star-forming regions. Most 
notably, $\mathrm{SO}$ and $\mathrm{SO}_{2}$ are well-known outflow tracers, which vary in brightness on cloud scales. In the work of Bockelée-Morvan et al. (2000), ISM observations were a compilation of data on the region L1157-B1 shocked by a nearby low-mass protostar and the hot cores associated with forming high-mass protostars $\mathrm{W} 3\left(\mathrm{H}_{2} \mathrm{O}\right)$, G34.3 + 0.15, and Orion KL (Hot Core and Compact Ridge). Observations of such a diverse set of targets are sensitive to different spatial scales and are likely to probe several different components of star-forming systems simultaneously. The correlations seen in this work have been strengthened in the case of CHO-bearing molecules, in particular for $\mathrm{CO}$ and the estimate for $\mathrm{H}_{2} \mathrm{O}$, most likely due to the probing of identical spatial scales by the ALMA data on IRAS 16293-2422. This may also be the reason for the reduction of deviation from the linear correlation for $\mathrm{HNCO}$ and $\mathrm{HC}_{3} \mathrm{~N}$ in the $\mathrm{N}$-bearing family.

When exploring the correlations between cometary and ISM molecules, Bockelée-Morvan et al. (2000) used $\mathrm{CH}_{3} \mathrm{OH}$ to scale quantities for $\mathrm{CHO}$-bearing molecules and $\mathrm{HCN}$ for $\mathrm{N}$ - and $\mathrm{S}$ bearing species. Hence, $\mathrm{CH}_{3} \mathrm{OH}$ was the only chemically relevant scaling factor used. The choice of normalizing by $\mathrm{HCN}$ for $\mathrm{N}$ and S-bearing species was justified on the basis of the comparable $\mathrm{D} / \mathrm{H}$ ratio as measured in $\mathrm{CH}_{3} \mathrm{OH}$ and $\mathrm{HCN}$. Now, $20 \mathrm{yr}$ later, it is not so clear whether this is something that holds true for protostellar sources in general. HCN was also chosen due to its high abundance (or production rate) in Hale-Bopp at that time. Beyond the argument of spatial scales of ISM observations (discussed in the above paragraph, and as $\mathrm{HCN}$ is expected to thermally desorb on envelope-scales at cool temperatures), the newly uncovered correlation in S-bearing molecules may have emerged thanks to the choice of a more representative reference molecule (that is $\mathrm{CH}_{3} \mathrm{SH}$ ).

Formamide can be classified as either a $\mathrm{CHO}$ - or an N-bearing family member. However, it appears to lie closer to the linear correlation seen in the N-bearing species, while it tends to be more of an outlier in the CHO-bearing family. This may suggest that it is more strongly chemically related to $\mathrm{CH}_{3} \mathrm{CN}$ and the $\mathrm{N}$-bearing molecules, rather than $\mathrm{CH}_{3} \mathrm{OH}$ and the $\mathrm{CHO}$-bearing species. Formamide has already been suggested to be closely related to HNCO based on observational data (Bisschop et al. 2007; López-Sepulcre et al. 2015; Coutens et al. 2016). Laboratory data are indicating that formamide is a result of combined NO hydrogenation and photolysis in CO-rich ices, and therefore linked to the formation of HNCO (Noble et al. 2015; Fedoseev et al. 2015, 2016). Theoretical calculations have suggested that the link of HNCO with formamide may stem from the two molecules reacting analogously in a physical environment at a certain temperature, but not necessarily implying a chemical connection (Quénard et al. 2018b).

\subsection{Contributions from disc chemistry}

The emission from gas-phase molecules that are observed with ALMA in IRAS 16293-2422 B is assumed to be directly representative of the ices that are being transported into the 'disc-like' structure around the protostar. However, this may not necessarily be the case as a result of the precise transport mechanisms of planetesimals and cometesimals into a protoplanetary disc. The exact location of formation of such bodies cannot be observed directly, and neither can their route into a disc. Only theoretical studies can probe these physical processes and suggest that the enhanced dust temperatures and UV fluxes do chemically alter the volatiles between the prestellar and protoplanetary disc phases during infall (Visser et al. 2009; Visser, Doty \& van Dishoeck 2011; Drozdovskaya et al. 2014, 2016; Hincelin et al. 2016; Yoneda et al. 2016). However, what exactly transpires at the disc-envelope boundary still remains unclear, for example. On the other hand, once inside the disc, icy volatiles that are locked up in sufficiently large cometesimals and that remain in the outer parts of the protoplanetary disc for the rest of the time, would no longer be affected by disc chemical processes. Hence, implying that the bulk composition of cometesimals could still be pristine disc-composing materials.

Direct observations of ices in protostellar systems and protoplanetary discs would be more directly comparable to cometary volatiles. Unfortunately, solid state observations have only been possible thanks to unique configurations in a handful of somewhat older (Class II) discs with only $\mathrm{H}_{2} \mathrm{O}, \mathrm{CO}, \mathrm{OCN}^{-}, \mathrm{OCS}$, and tentatively HDO being detected so far (Pontoppidan et al. 2005; Honda et al. 2009; Aikawa et al. 2012; Terada et al. 2012; Terada \& Tokunaga 2012; McClure et al. 2015). The James Webb Space Telescope (JWST) is expected to make much greater progress on this topic (for example within the framework of the Mid-Infrared Instrument (MIRI) European Consortium (EC) 'Protostars Survey' Guaranteed Time Observations (GTO) program, PI: Ewine F. van Dishoeck, and the 'IceAge: Chemical Evolution of Ices during Star Formation' Director's Discretionary Early Release Science (DDERS) program, McClure et al. 2018). Such comparisons will be the subject of future work; however, they will always be limited to the most abundant icy volatiles due to the need for large quantities of individual molecules to generate detectable absorption features. The full chemical inventory of such diverse sets of molecules is only possible in the gas phase with facilities such as ALMA.

\section{CONCLUSIONS}

In the quest to identify the ingredients that are needed to form Solar-like systems, a comparative study has been carried out between IRAS 16293-2422 B and 67P/C-G. IRAS 16293-2422 is an embedded low-mass binary protostellar system that is thought to be analogous to the youngest stages of formation of our Solar System. Source B is favourably positioned in the sky for a complete and quantitative chemical inventory with observations carried out by ALMA with the PILS survey on protoplanetary disc-scales (Jørgensen et al. 2016). 67P/C-G is a Jupiter-family comet that has been monitored continuously for more than $2 \mathrm{yr}$ by the instruments of the Rosetta mission allowing an unprecedented characterization of its composition and the first-time derivation of bulk cometary molecular abundances (Rubin et al. 2019). In this paper, the most complete molecular inventory to date of both targets has been compared in terms of relative abundances. The main conclusions are as follows.

(i) Abundances of $\mathrm{CHO}-, \mathrm{N}-$, and $\mathrm{S}$-bearing molecules display correlations between the protostellar IRAS 16293-2422 B and the cometary $67 \mathrm{P} / \mathrm{C}-\mathrm{G}$ volatiles relative to $\mathrm{CH}_{3} \mathrm{OH}, \mathrm{CH}_{3} \mathrm{CN}$, and $\mathrm{CH}_{3} \mathrm{SH}$, respectively, with some scatter. Tentative correlations between $\mathrm{P}$ - and Cl-bearing molecules relative to $\mathrm{CH}_{3} \mathrm{OH}$ are inferred. This suggests preservation of prestellar and protostellar volatiles into cometary bodies upon some degree of chemical alteration.

(ii) Cometary relative abundances (as measured for 67P/C-G) tend to be higher than protostellar quantities (as observed in IRAS 16293-2422 B) for CHO- and N-bearing species, which may indicate either that volatile molecules are destroyed near the protostar before entry into the protoplanetary disc or that more have been produced by the time of incorporation into the comet. It cannot be excluded that this may stem from variations of solely 
the reference molecules $\left(\mathrm{CH}_{3} \mathrm{OH}\right.$ and $\left.\mathrm{CH}_{3} \mathrm{CN}\right)$ between comets and protostellar regions.

(iii) Links between Hale-Bopp's and ISM volatiles have been confirmed for the case of $67 \mathrm{P} / \mathrm{C}-\mathrm{G}$ for $\mathrm{CHO}$ - and N-bearing molecules on protoplanetary disc-scales. For S-bearing species these may have been missed previously for Hale-Bopp due to the use of an unrepresentative reference molecule, the importance of distributed sources for S-bearing volatiles, the snap-shot nature of cometary ground-based observations or the low spatial resolution ISM data points that encompass many structures of star-forming regions simultaneously.

(iv) The volatile composition of cometesimals and planetesimals is partially inherited from the pre- and protostellar phases of evolution.

A more direct comparison with bulk cometary volatiles could be achieved by probing protoplanetary disc ices with data from future mission such as the JWST; however, this would always be limited to only the most-abundant icy species, as minor constituents would not generate observable absorption features. The legacy of the detailed in situ study of a comet as was achieved with the Rosetta mission should be extended in the future through analogous missions to comets of different dynamic origins and other small bodies of our Solar System.

\section{ACKNOWLEDGEMENTS}

This work is supported by the Swiss National Science Foundation (SNSF) Ambizione grant 180079, the Center for Space and Habitability (CSH) Fellowship, and the IAU Gruber Foundation Fellowship. MR acknowledges the support of the state of Bern and the SNSF (200020_182418). JKJ is supported by the European Research Council (ERC) under the European Union's Horizon 2020 research and innovation programme through ERC Consolidator Grant 'S4F' (grant agreement No. 646908). Research at Centre for Star and Planet Formation is funded by the Danish National Research Foundation.

The authors would like to acknowledge the contributions to this work of the entire PILS and ROSINA teams, as well as input of Holger S. P. Müller with regards to the spectroscopy of glycine, and useful discussions with Nadia Murillo and Matthijs van der Wiel about binary protostellar sources.

This paper makes use of the following ALMA data: ADS/JAO.ALMA\#2013.1.00278.S, ADS/JAO.ALMA\#2012.1.00712.S, ADS/JAO.ALMA\#2016.1.01150.S., ADS/JAO.ALMA\#2011.0.00007.SV, ADS/JAO.ALMA\#2013.1.00061.S, ADS/JAO.ALMA\#2017.1.00518.S, and ADS/JAO.ALMA\#2015.1.01193.S. ALMA is a partnership of ESO (representing its member states), NSF (USA) and NINS (Japan), together with NRC (Canada), MOST and ASIAA (Taiwan), and KASI (Republic of Korea), in cooperation with the Republic of Chile. The Joint ALMA Observatory is operated by ESO, AUI/NRAO, and NAOJ.

\section{REFERENCES}

Aikawa Y. et al., 2012, A\&A, 538, A57

ALMA Partnership, 2015, ApJ, 808, L3

Altwegg K., Balsiger H., Fuselier S. A., 2019, ARA\&A, 57, 113

Altwegg K. et al., 2016, Sci. Adv., 2, e1600285
Altwegg K. et al., 2017a, Phil. Trans. R. Soc. A, 375, 20160253

Altwegg K. et al., 2017b, MNRAS, 469, S130

Alves F. O., Vlemmings W. H. T., Girart J. M., Torrelles J. M., 2012, A\&A, 542, A 14

Andron I., Gratier P., Majumdar L., Vidal T. H. G., Coutens A., Loison J.-C., Wakelam V., 2018, MNRAS, 481, 5651

Ansdell M. et al., 2016, ApJ, 828, 46

A'Hearn M. F., 2011, ARA\&A, 49, 281

Bacmann A., Faure A., 2016, A\&A, 587, A130

Bacmann A. et al., 2010, A\&A, 521, L42

Balsiger H. et al., 2007, Space Sci. Rev., 128, 745

Barucci M. A. et al., 2016, A\&A, 595, A102

Baryshev A. M. et al., 2015, A\&A, 577, A129

Birch S. P. D. et al., 2017, MNRAS, 469, S50

Bisschop S. E., Jørgensen J. K., Bourke T. L., Bottinelli S., van Dishoeck E. F., 2008, A\&A, 488, 959

Bisschop S. E., Jørgensen J. K., van Dishoeck E. F., de Wachter E. B. M., 2007, A\&A, 465, 913

Biver N. et al., 2014, A\&A, 566, L5

Biver N. et al., 2015, A\&A, 583, A3

Blake G. A., van Dishoeck E. F., Jansen D. J., Groesbeck T. D., Mundy L. G., 1994, ApJ, 428, 680

Bockelée-Morvan D. et al., 2000, A\&A, 353, 1101

Bockelée-Morvan D. et al., 2015a, Space Sci. Rev., 197, 47

Bockelée-Morvan D. et al., 2015b, A\&A, 583, A6

Bockelée-Morvan D. et al., 2016, MNRAS, 462, S170

Bosman A. D., Walsh C., van Dishoeck E. F., 2018, A\&A, 618, A182

Bottinelli S., Wakelam V., Caux E., Vastel C., Aikawa Y., Ceccarelli C., 2014, MNRAS, 441, 1964

Bottinelli S. et al., 2004, ApJ, 617, L69

Brünken S. et al., 2014, Nature, 516, 219

Butner H. M., Charnley S. B., Ceccarelli C., Rodgers S. D., Pardo J. R., Parise B., Cernicharo J., Davis G. R., 2007, ApJ, 659, L137

Calcutt H. et al., 2018a, A\&A, 617, A95

Calcutt H. et al., 2018b, A\&A, 616, A90

Calmonte U. et al., 2016, MNRAS, 462, S253

Capria M. T. et al., 2017, MNRAS, 469, S685

Castets A., Ceccarelli C., Loinard L., Caux E., Lefloch B., 2001, A\&A, 375 , 40

Caux E. et al., 2011, A\&A, 532, A23

Cazaux S., Tielens A. G. G. M., Ceccarelli C., Castets A., Wakelam V., Caux E., Parise B., Teyssier D., 2003, ApJ, 593, L51

Ceccarelli C., Castets A., Caux E., Hollenbach D., Loinard L., Molinari S., Tielens A. G. G. M., 2000a, A\&A, 355, 1129

Ceccarelli C., Castets A., Loinard L., Caux E., Tielens A. G. G. M., 1998a, A\&A, 338, L43

Ceccarelli C., Caux E., Wolfire M., Rudolph A., Nisini B., Saraceno P., White G. J., 1998c, A\&A, 331, L17

Ceccarelli C., Haas M. R., Hollenbach D. J., Rudolph A. L., 1997, ApJ, 476, 771

Ceccarelli C., Loinard L., Castets A., Faure A., Lefloch B., 2000b, A\&A, 362,1122

Ceccarelli C., Loinard L., Castets A., Tielens A. G. G. M., Caux E., 2000c, A\&A, 357, L9

Ceccarelli C., Loinard L., Castets A., Tielens A. G. G. M., Caux E., Lefloch B., Vastel C., 2001, A\&A, 372, 998

Ceccarelli C., Maret S., Tielens A. G. G. M., Castets A., Caux E., 2003, A\&A, 410, 587

Ceccarelli C. et al., 1998b, A\&A, 331, 372

Ceccarelli C. et al., 2010, A\&A, 521, L22

Chandler C. J., Brogan C. L., Shirley Y. L., Loinard L., 2005, ApJ, 632, 371

Choi M., Panis J.-F., Evans N. J., II, 1999, ApJS, 122, 519

Cochran A. L. et al., 2015, Space Sci. Rev., 197, 9

Colom P., Lekht E. E., Pashchenko M. I., Rudnitskii G. M., Tolmachev A. M., 2016, Astron. Rep., 60, 730

Cottin H., Fray N., 2008, Space Sci. Rev., 138, 179

Coudert L. H., Margulès L., Vastel C., Motiyenko R., Caux E., Guillemin J.-C., 2019, A\&A, 624, A70 
Coutens A., Zakharenko O., Lewen F., Jørgensen J. K., Schlemmer S., Müller H. S. P., 2019b, A\&A, 623, A93

Coutens A. et al., 2012, A\&A, 539, A132

Coutens A. et al., 2013, A\&A, 553, A75

Coutens A. et al., 2016, A\&A, 590, L6

Coutens A. et al., 2018, A\&A, 612, A107

Coutens A. et al., 2019a, A\&A, 623, L13

Davidsson B. J. R. et al., 2016, A\&A, 592, A63

Dello Russo N., Kawakita H., Vervack R. J., Weaver H. A., 2016, Icarus, 278, 301

Demyk K., Bottinelli S., Caux E., Vastel C., Ceccarelli C., Kahane C., Castets A., 2010, A\&A, 517, A17

De Sanctis M. C. et al., 2015, Nature, 525, 500

Dhooghe F. et al., 2017, MNRAS, 472, 1336

Drozdovskaya M. N., Walsh C., van Dishoeck E. F., Furuya K., Marboeuf U., Thiabaud A., Harsono D., Visser R., 2016, MNRAS, 462, 977

Drozdovskaya M. N., Walsh C., Visser R., Harsono D., van Dishoeck E. F., 2014, MNRAS, 445, 913

Drozdovskaya M. N. et al., 2018, MNRAS, 476, 4949

Dzib S. A. et al., 2018, A\&A, 614, A20

Ehrenfreund P., Charnley S. B., 2000, ARA\&A, 38, 427

Endres C. P., Schlemmer S., Schilke P., Stutzki J., Müller H. S. P., 2016, J. Mol. Spectrosc., 327, 95

Favre C., Jørgensen J. K., Field D., Brinch C., Bisschop S. E., Bourke T. L., Hogerheijde M. R., Frieswijk W. W. F., 2014, ApJ, 790, 55

Fayolle E. C. et al., 2017, Nat. Astron., 1, 703

Fedoseev G., Chuang K.-J., Ioppolo S., Qasim D., van Dishoeck E. F., Linnartz H., 2017, ApJ, 842, 52

Fedoseev G., Chuang K.-J., van Dishoeck E. F., Ioppolo S., Linnartz H., 2016, MNRAS, 460, 4297

Fedoseev G., Ioppolo S., Zhao D., Lamberts T., Linnartz H., 2015, MNRAS, 446, 439

Feldman P. D. et al., 2016, ApJ, 825, L8

Filacchione G. et al., 2016a, Nature, 529, 368

Filacchione G. et al., 2016b, Science, 354, 1563

Filacchione G. et al., 2019, Space Sci. Rev., 215, 19

Fornasier S. et al., 2016, Science, 354, 1566

Fuchs G. W., Cuppen H. M., Ioppolo S., Romanzin C., Bisschop S. E., Andersson S., van Dishoeck E. F., Linnartz H., 2009, A\&A, 505, 629

Garay G., Mardones D., Rodríguez L. F., Caselli P., Bourke T. L., 2002, ApJ, 567, 980

Garrod R. T., Weaver S. L. W., Herbst E., 2008, ApJ, 682, 283

Gasc S. et al., 2017, MNRAS, 469, S108

Girart J. M., Estalella R., Palau A., Torrelles J. M., Rao R., 2014, ApJ, 780, L11

Greenberg J. M., Li A., 1999, Space Sci. Rev., 90, 149

Hansen K. C. et al., 2016, MNRAS, 462, S491

Harju J. et al., 2017, ApJ, 840, 63

Hernández-Gómez A., Sahnoun E., Caux E., Wiesenfeld L., Loinard L., Bottinelli S., Hammami K., Menten K. M., 2019b, MNRAS, 483, 2014

Hernández-Gómez A. et al., 2019a, ApJ, 875, 94

Hily-Blant P. et al., 2010, A\&A, 521, L52

Hincelin U., Commerçon B., Wakelam V., Hersant F., Guilloteau S., Herbst E., 2016, ApJ, 822, 12

Hirano N., Liu F.-c., 2014, ApJ, 789, 50

Hirano N., Mikami H., Umemoto T., Yamamoto S., Taniguchi Y., 2001, ApJ, 547, 899

Hjalmarson Å. et al., 2003, A\&A, 402, L39

Honda M. et al., 2009, ApJ, 690, L110

Huang H.-C., Kuan Y.-J., Charnley S. B., Hirano N., Takakuwa S., Bourke T. L., 2005, Adv. Space Res., 36, 146

Hässig M. et al., 2015, Science, 347, aaa0276

Imai H., Iwata T., Miyoshi M., 1999, PASJ, 51, 473

Jaber Al-Edhari A. et al., 2017, A\&A, 597, A40

Jacobsen S. K., Jørgensen J. K., Di Francesco J., Evans N. J., II, Choi M., Lee J.-E., 2019, A\&A, 629, A29

Jacobsen S. K. et al., 2018, A\&A, 612, A72

Jorda L. et al., 2016, Icarus, 277, 257
Jutzi M., Asphaug E., 2015, Science, 348, 1355

Jutzi M., Benz W., 2017, A\&A, 597, A62

Jutzi M., Benz W., Toliou A., Morbidelli A., Brasser R., 2017, A\&A, 597, A61

Jørgensen J. K., Bourke T. L., Nguyen Luong Q., Takakuwa S., 2011, A\&A, $534, \mathrm{~A} 100$

Jørgensen J. K., Favre C., Bisschop S. E., Bourke T. L., van Dishoeck E. F., Schmalzl M., 2012, ApJ, 757, L4

Jørgensen J. K., Schöier F. L., van Dishoeck E. F., 2004, A\&A, 416, 603

Jørgensen J. K. et al., 2016, A\&A, 595, A117

Jørgensen J. K. et al., 2018, A\&A, 620, A170

Kahane C., Ceccarelli C., Faure A., Caux E., 2013, ApJ, 763, L38

Keller H. U., Mottola S., Skorov Y., Jorda L., 2015, A\&A, 579, L5

Keller H. U. et al., 2017, MNRAS, 469, S357

Kofman W. et al., 2015, Science, 349, 283

Kramer T., Laeuter M., Hviid S., Jorda L., Keller H. U., Kührt E., 2018, preprint (arXiv: 1812.01490)

Kristensen L. E., Klaassen P. D., Mottram J. C., Schmalzl M., Hogerheijde M. R., 2013, A\&A, 549, L6

Kuan Y.-J. et al., 2004, ApJ, 616, L27

Lamberts T., 2018, A\&A, 615, L2

Lee S. et al., 2015, A\&A, 583, A5

Ligterink N. F. W., Terwisscha van Scheltinga J., Taquet V., Jørgensen J. K., Cazaux S., van Dishoeck E. F., Linnartz H., 2018b, MNRAS, 480, 3628

Ligterink N. F. W. et al., 2017, MNRAS, 469, 2219

Ligterink N. F. W. et al., 2018a, A\&A, 619, A28

Lindberg J. E., Charnley S. B., Jørgensen J. K., Cordiner M. A., Bjerkeli P., 2017, ApJ, 835, 3

Lis D. C., Gerin M., Phillips T. G., Motte F., 2002, ApJ, 569, 322

Lis D. C., Wootten H. A., Gerin M., Pagani L., Roueff E., van der Tak F. F. S., Vastel C., Walmsley C. M., 2016, ApJ, 827, 133

Liu H. B., Hasegawa Y., Ching T.-C., Lai S.-P., Hirano N., Rao R., 2018, A\&A, 617, A3

Loinard L., Castets A., Ceccarelli C., Tielens A. G. G. M., Faure A., Caux E., Duvert G., 2000, A\&A, 359, 1169

Luspay-Kuti A. et al., 2015, A\&A, 583, A4

Lykke J. M. et al., 2017, A\&A, 597, A53

López-Sepulcre A. et al., 2015, MNRAS, 449, 2438

Majumdar L., Gratier P., Andron I., Wakelam V., Caux E., 2017, MNRAS, 467,3525

Majumdar L., Gratier P., Vidal T., Wakelam V., Loison J.-C., Hickson K. M., Caux E., 2016, MNRAS, 458, 1859

Majumdar L., Gratier P., Wakelam V., Caux E., Willacy K., Ressler M. E., 2018, MNRAS, 477, 525

Manigand S. et al., 2019, A\&A, 623, A69

Marcelino N., Brünken S., Cernicharo J., Quan D., Roueff E., Herbst E., Thaddeus P., 2010, A\&A, 516, A105

Marshall D. W. et al., 2017, A\&A, 603, A87

Martín-Doménech R., Jiménez-Serra I., Muñoz Caro G. M., Müller H. S. P., Occhiogrosso A., Testi L., Woods P. M., Viti S., 2016, A\&A, 585, A112

Martín-Doménech R., Rivilla V. M., Jiménez-Serra I., Quénard D., Testi L., Martín-Pintado J., 2017, MNRAS, 469, 2230

Massironi M. et al., 2015, Nature, 526, 402

Matonti C. et al., 2019, Nat. Geosci., 12, 157

McClure M. K. et al., 2015, ApJ, 799, 162

McClure M. K. et al., 2018, in American Astronomical Society Meeting Abstracts, Vol. 232, American Astronomical Society Meeting Abstracts \#232. Denver, CO, p. 302.03

Menten K. M., Serabyn E., Guesten R., Wilson T. L., 1987, A\&A, 177, L57

Migliorini A. et al., 2016, A\&A, 589, A45

Mizuno A., Fukui Y., Iwata T., Nozawa S., Takano T., 1990, ApJ, 356, 184

Mumma M. J., Charnley S. B., 2011, ARA\&A, 49, 471

Mundy L. G., Wilking B. A., Myers S. T., 1986, ApJ, 311, L75

Mundy L. G., Wootten A., Wilking B. A., Blake G. A., Sargent A. I., 1992, ApJ, 385, 306

Mundy L. G., Wootten H. A., Wilking B. A., 1990, ApJ, 352, 159

Murillo N. M., van Dishoeck E. F., Tobin J. J., Mottram J. C., Karska A., 2018a, A\&A, 620, A30 
Murillo N. M., van Dishoeck E. F., van der Wiel M. H. D., Jørgensen J. K., Drozdovskaya M. N., Calcutt H., Harsono D., 2018b, A\&A, 617, A120

Müller H. S. P., Schlöder F., Stutzki J., Winnewisser G., 2005, J. Mol. Struct., 742,215

Müller H. S. P., Thorwirth S., Roth D. A., Winnewisser G., 2001, A\&A, 370, L49

Narayanan G., Walker C. K., Buckley H. D., 1998, ApJ, 496, 292

Noble J. A. et al., 2015, A\&A, 576, A91

Öberg K. I., Boogert A. C. A., Pontoppidan K. M., van den Broek S., van Dishoeck E. F., Bottinelli S., Blake G. A., Evans N. J., II, 2011, ApJ, 740,109

Oya Y., Sakai N., López-Sepulcre A., Watanabe Y., Ceccarelli C., Lefloch B., Favre C., Yamamoto S., 2016, ApJ, 824, 88

Oya Y. et al., 2018, ApJ, 854, 96

Pajola M. et al., 2017, Nat. Astron., 1, 0092

Parise B., Castets A., Herbst E., Caux E., Ceccarelli C., Mukhopadhyay I., Tielens A. G. G. M., 2004, A\&A, 416, 159

Parise B. et al., 2002, A\&A, 393, L49

Parise B. et al., 2005, A\&A, 431, 547

Parise B. et al., 2012, A\&A, 542, L5

Peng R., Yoshida H., Chamberlin R. A., Phillips T. G., Lis D. C., Gerin M., 2010, ApJ, 723, 218

Persson M. V., Jørgensen J. K., van Dishoeck E. F., 2013, A\&A, 549, L3

Persson M. V., Jørgensen J. K., van Dishoeck E. F., Harsono D., 2014, A\&A, 563, A74

Persson M. V. et al., 2018, A\&A, 610, A54

Pickett H. M., Poynter R. L., Cohen E. A., Delitsky M. L., Pearson J. C., Müller H. S. P., 1998, J. Quant. Spec. Radiat. Transf., 60, 883

Pineda J. E. et al., 2012, A\&A, 544, L7

Pontoppidan K. M., Dullemond C. P., van Dishoeck E. F., Blake G. A., Boogert A. C. A., Evans N. J., II, Kessler-Silacci J. E., Lahuis F., 2005, ApJ, 622, 463

Pontoppidan K. M., Salyk C., Bergin E. A., Brittain S., Marty B., Mousis O., Öberg K. I., 2014, in Henrik B., Klessen R. S., Dullemond C. P., Henning T., eds, Protostars and Planets VI, Volatiles in Protoplanetary Disks. Univ. Arizona Press, Tucson, AZ, p. 363

Pätzold M. et al., 2016, Nature, 530, 63

Quénard D., Bottinelli S., Caux E., Wakelam V., 2018a, MNRAS, 477, 5312

Quénard D., Jiménez-Serra I., Viti S., Holdship J., Coutens A., 2018b, MNRAS, 474, 2796

Rao R., Girart J. M., Marrone D. P., Lai S.-P., Schnee S., 2009, ApJ, 707, 921

Remijan A., Snyder L. E., Friedel D. N., Liu S.-Y., Shah R. Y., 2003, ApJ, 590,314

Remijan A. J., Hollis J. M., 2006, ApJ, 640, 842

Rice T. S., Bergin E. A., Jørgensen J. K., Wampfler S. F., 2018, ApJ, 866 , 156

Richard C. et al., 2013, A\&A, 552, A117

Rivilla V. M., Beltrán M. T., Vasyunin A., Caselli P., Viti S., Fontani F., Cesaroni R., 2019, MNRAS, 483, 806

Rodríguez L. F., Loinard L., D’Alessio P., Wilner D. J., Ho P. T. P., 2005 , ApJ, 621, L133

Rubin M. et al., 2018, Sci. Adv., 4, eaar6297

Rubin M. et al., 2019, MNRAS, 489, 594

Sadavoy S. I. et al., 2018, ApJ, 869, 115

Schloerb F. P. et al., 2015, A\&A, 583, A29

Schuhmann M.et al., 2019, American Astronomical Society Meeting Abstracts, 51, 208.01

Schwartz S. R., Michel P., Jutzi M., Marchi S., Zhang Y., Richardson D. C., 2018, Nat. Astron., 2, 379

Schöier F. L., Jørgensen J. K., van Dishoeck E. F., Blake G. A., 2002, A\&A, 390,1001

Schöier F. L., Jørgensen J. K., van Dishoeck E. F., Blake G. A., 2004, A\&A, 418,185

Shiao Y.-S. J., Looney L. W., Remijan A. J., Snyder L. E., Friedel D. N., 2010, ApJ, 716, 286

Shu F. H., Adams F. C., Lizano S., 1987, ARA\&A, 25, 23
Stark R. et al., 2004, ApJ, 608, 341

Stern S. A. et al., 2019, Science, 364, aaw9771

Takakuwa S., Kamazaki T., 2011, PASJ, 63, 921

Takakuwa S. et al., 2007, ApJ, 662, 431

Taquet V., López-Sepulcre A., Ceccarelli C., Neri R., Kahane C., Charnley S. B., 2015, ApJ, 804, 81

Taquet V. et al., 2018, A\&A, 618, A11

Terada H., Tokunaga A. T., 2012, ApJ, 753, 19

Terada H. et al., 2012, AJ, 144, 175

Tielens A. G. G. M., Hagen W., 1982, A\&A, 114, 245

Tobin J. J. et al., 2016, ApJ, 818, 73

van der Wiel M. H. D. et al., 2019, A\&A, 626, A93

van Dishoeck E. F., Blake G. A., Jansen D. J., Groesbeck T. D., 1995, ApJ, 447,760

van Kempen T. A. et al., 2009, A\&A, 507, 1425

Vastel C. et al., 2010, A\&A, 521, L31

Vidal T. H. G., Loison J.-C., Jaziri A. Y., Ruaud M., Gratier P., Wakelam V., 2017, MNRAS, 469, 435

Vigorito A., Calabrese C., Melandri S., Caracciolo A., Mariotti S., Giannetti A., Massardi M., Maris A., 2018, A\&A, 619, A140

Vincent J.-B. et al., 2015, Nature, 523, 63

Vincent J.-B. et al., 2016, MNRAS, 462, S184

Visser R., Doty S. D., van Dishoeck E. F., 2011, A\&A, 534, A132

Visser R., van Dishoeck E. F., Doty S. D., Dullemond C. P., 2009, A\&A, 495,881

Wakelam V., Castets A., Ceccarelli C., Lefloch B., Caux E., Pagani L., 2004, A\&A, 413, 609

Walker C. K., Carlstrom J. E., Bieging J. H., 1993, ApJ, 402, 655

Walker C. K., Carlstrom J. E., Bieging J. H., Lada C. J., Young E. T., 1990, ApJ, 364, 173

Walker C. K., Lada C. J., Young E. T., Maloney P. R., Wilking B. A., 1986, ApJ, 309, L47

Walker C. K., Lada C. J., Young E. T., Margulis M., 1988, ApJ, 332, 335

Walker C. K., Maloney P. R., Serabyn E., 1994, ApJ, 437, L127

Wampfler S. F., Jørgensen J. K., Bizzarro M., Bisschop S. E., 2014, A\&A, 572, A24

Wilking B. A., Claussen M. J., 1987, ApJ, 320, L133

Wootten A., 1989, ApJ, 337, 858

Wootten A., Loren R. B., 1987, ApJ, 317, 220

Yeh S. C. C., Hirano N., Bourke T. L., Ho P. T. P., Lee C.-F., Ohashi N., Takakuwa S., 2008, ApJ, 675, 454

Yoneda H., Tsukamoto Y., Furuya K., Aikawa Y., 2016, ApJ, 833, 105

Young E. T., Lada C. J., Wilking B. A., 1986, ApJ, 304, L45

Yildız U. A. et al., 2013, A\&A, 556, A89

Yildız U. A. et al., 2015, A\&A, 576, A109

Zakharenko O., Lewen F., Ilyushin V. V., Drozdovskaya M. N., Jørgensen J. K., Schlemmer S., Müller H. S. P., 2019, A\&A, 621, A114

Zapata L. A., Loinard L., Rodríguez L. F., Hernández-Hernández V., Takahashi S., Trejo A., Parise B., 2013, ApJ, 764, L14

Zeng S., Quénard D., Jiménez-Serra I., Martín-Pintado J., Rivilla V. M., Testi L., Martín-Doménech R., 2019, MNRAS, 484, L43

Zhou Y. et al., 2018, preprint (arXiv:1807.00986)

\section{APPENDIX A: NEWLY DETERMINED COLUMN DENSITIES IN IRAS 16293-2422 B}

This study required a full chemical inventory of IRAS 16293$2422 \mathrm{~B}$, including some molecules for which column densities (or at least upper limits on them) have not been previously published, and hence, had to be newly derived. These are presented in this appendix. The synthetic spectra have been generated with custom IDL routines under the assumption of local thermal equilibrium (LTE), which has been justified for the case of methanol in section 5.1 of Jørgensen et al. (2016). The spectroscopic data stem from the 
Cologne Database of Molecular Spectroscopy (CDMS; Müller et al. 2001, 2005; Endres et al. 2016).

\section{A1 CO}

For a highly abundant species, such as $\mathrm{CO}$, line optical thickness is a severe problem for determining the column densities, even of the isotopologues. The PILS Band 7 range covers the $J=3-2$ transitions of the four main isotopologues of $\mathrm{CO}\left({ }^{12} \mathrm{CO},{ }^{13} \mathrm{CO}\right.$, $\mathrm{C}^{18} \mathrm{O}$ and $\mathrm{C}^{17} \mathrm{O}$ ); while the transitions of the less abundant doubly substituted variants and the vibrationally excited states fall at lower frequencies outside of this range. The two rarer variants of these four, $\mathrm{C}^{18} \mathrm{O}$, and $\mathrm{C}^{17} \mathrm{O}$, both show inverse $\mathrm{P}$ Cygni profiles towards the one-beam offset position, which is an indication of significant optical depth. Consequently, only a ball-park number for the $\mathrm{CO}$ column density can be derived: for $\mathrm{C}^{17} \mathrm{O}$, the blue emission part of the line profiles suggests temperatures in the $100-150 \mathrm{~K}$ range with a column density of $5 \times 10^{16} \mathrm{~cm}^{-2}$. A higher column density can also fit the $\mathrm{C}^{17} \mathrm{O}$ transition, if a lower excitation temperature is adopted. However, that would be at odds with the $\mathrm{C}^{18} \mathrm{O} J=3-2$ transition that is then severely underproduced.

The derived $\mathrm{C}^{17} \mathrm{O}$ column density would imply an overall $\mathrm{CO}$ column density of $\sim 1 \times 10^{20} \mathrm{~cm}^{-2}$, i.e. an order of magnitude above that of $\mathrm{CH}_{3} \mathrm{OH}$. Adopting the lower limit for the $\mathrm{H}_{2}$ column density based on the dust continuum emission (Jørgensen et al. 2016), the upper limit to the $\mathrm{CO}$ abundance relative to $\mathrm{H}_{2}$, is $2 \times 10^{-5}$. These relative $\mathrm{CH}_{3} \mathrm{OH} / \mathrm{CO}$ and $\mathrm{CO} / \mathrm{H}_{2}$ abundances are not unreasonable, e.g. in comparison to typical ice measurements in star-forming regions. However, due to the above uncertainties, the derived value is at best only accurate to within a factor of a few.

\section{A2 HCN and HNC}

Like for $\mathrm{CO}$, the lines of the $\mathrm{HCN}$ and $\mathrm{HNC}$ are strongly affected by optical depth issues. The PILS Band 7 data cover the $\mathrm{HC}^{15} \mathrm{~N} \mathrm{~J}=$ $4-3$ transition at $344.2 \mathrm{GHz}$. If an excitation temperature of $120 \mathrm{~K}$ is assumed, this transition is fit by a column density of $2.5 \times 10^{14} \mathrm{~cm}^{-2}$ with the emission being marginally optically thick. This corresponds to a column density of $5 \times 16 \mathrm{~cm}^{-2}$ for the main isotopologue. The PILS Band 7 data also cover the $J=4-3$ transition of $\mathrm{DC}^{15} \mathrm{~N}$ and a tentative assignment can be made at an implied column density of $7.5 \times 10^{12} \mathrm{~cm}^{-2}$. This would correspond to a $\mathrm{D} / \mathrm{H}$ ratio of about 3 percent, which is in-line with other measurements of D/H ratios from PILS (e.g. Drozdovskaya et al. 2018; Jørgensen et al. 2018). Conversely, if the HCN column density had been grossly underestimated, the $\mathrm{D} / \mathrm{H}$ ratio would need to be much lower for this species in particular. Finally, the PILS Band 7 data also cover two lines of vibrationally excited $\mathrm{HCN}$. If correctly assigned, these transitions would imply a column density of $3 \times 10^{17} \mathrm{~cm}^{-2}$, assuming an excitation temperature of $120 \mathrm{~K}$, while an assumed temperature of $200 \mathrm{~K}$ would lower this to $2 \times 10^{16} \mathrm{~cm}^{-2}$. Thus, for $\mathrm{HCN}$, the vibrationally excited lines correspond to the same regime as the isotopologues, but do not provide any stronger constraints.

For HNC, no emission is seen from either isotopologue or vibrationally excited states. A possible explanation is that HNC has a much more compact distribution than that of $\mathrm{HCN}$, causing its lines to be completely optically thick and fully quenched. It is thought that its column density cannot be higher than that of $\mathrm{HCN}$.

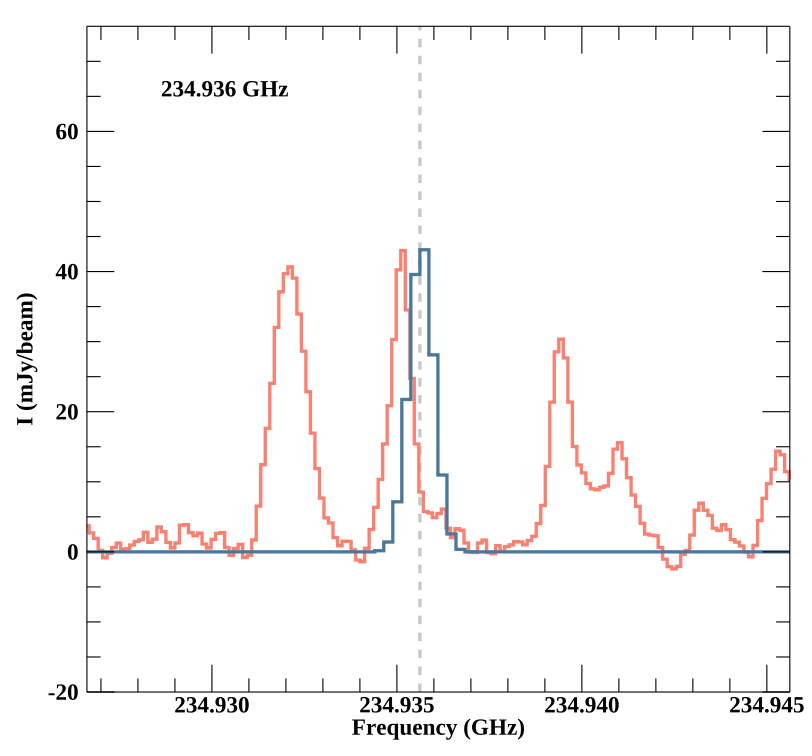

Figure A1. The detected $J=5-4$ line of PN in the $v=0$ state (CDMS entry $045511)$ with $E_{\mathrm{up}}=34 \mathrm{~K}$ and $A_{i j}=5.2 \times 10^{-4} \mathrm{~s}^{-1}$. The observed ALMA Band 6 spectrum convolved with a uniform circular restoring beam of 0 '.5 at the one-beam ( $\sim 70 \mathrm{au})$ offset from source B of IRAS 16293-2422 in the $\mathrm{SW}$ direction is in salmon. The turquoise line is the LTE fit for the transition, assuming a source size that is equal to the beam size of 0 "' 5 , full width halfmaximum (FWHM) of $1 \mathrm{~km} \mathrm{~s}^{-1}, T_{\mathrm{ex}}=125 \mathrm{~K}$, and $N=2.1 \times 10^{13} \mathrm{~cm}^{-2}$. The local standard of rest (LSR) velocity, $v_{\mathrm{LSR}}$, is assumed to be $2.7 \mathrm{~km} \mathrm{~s}^{-1}$ at this position.

Additionally, the detected $J=1-0$ lines of $\mathrm{HNC}$ and $\mathrm{HCN}$ lines in ALMA Band 3 were examined (project-id 2017.1.00518.S, PI: Ewine F. van Dishoeck, and project-id 2015.1.01193.S, PI: Víctor M. Rivilla), which indicate that the emission from these low excitation lines stems primarily from the circumbinary envelope scales. These data were obtained at a lower spatial resolution than the PILS observations ( $\sim 1$ arcsec versus 0.5), making it hard to conclusively derive the emitting regions. The analysis of HNC and HCN lines in ALMA Band 7 (PILS) suggests a much smaller column density for $\mathrm{HNC}$ than that of $\mathrm{HCN}$, as no emission is seen at frequencies corresponding to lines of vibrationally excited $\mathrm{HNC}$ and its isotopologues, while lines of vibrationally excited $\mathrm{HCN}$ and its isotopologues are detected. Consequently, an upper limit on $N(\mathrm{HNC})$ equal to $N(\mathrm{HCN})$ is assumed in this work.

\section{A3 PN}

IRAS 16293-2422 was observed in ALMA Band 6 under projectid 2016.1.01150.S (PI: Vianney Taquet) in Cycle 4. The data set appears to contain a detection of the $J=5-4$ line of PN in the $v=$ 0 state (Fig. A1) with $E_{\text {up }}=34 \mathrm{~K}$ and $A_{i j}=5.2 \times 10^{-4} \mathrm{~s}^{-1}$. This line was also covered in project-id: 2016.1.00457, PI: Yoko Oya; however, the data set has not been compared with that of projectid 2016.1.01150.S, PI: Vianney Taquet. The binary system was also observed in ALMA Band 9 under project-id 2011.0.00007.SV during the Science Verification phase. The data set covers the $J=$ $15-14$ line of PN in the $v=0$ state (Fig. A2) with $E_{\text {up }}=271 \mathrm{~K}$ and $A_{i j}=1.5 \times 10^{-2} \mathrm{~s}^{-1}$. However, this data set suffers from a poor baseline and a high noise level, making it hard to firmly constrain $T_{\mathrm{ex}}$ and $N$. It can only be used to verify that the column density derived based on the Band 6 line does not contradict the emission observed in Band 9. Using the spectra upon convolution with a uniform 


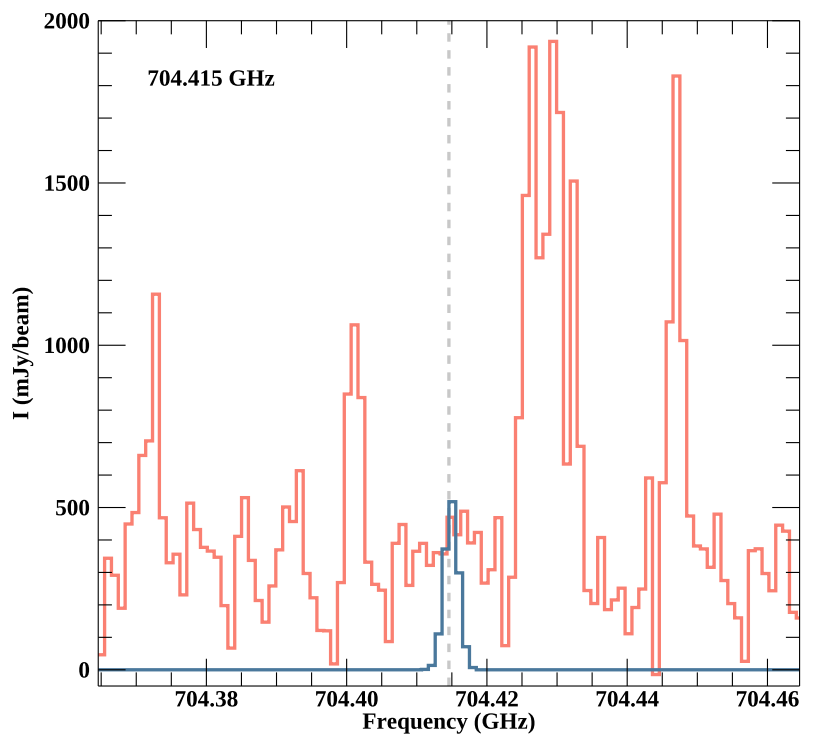

Figure A2. The covered $J=15-14$ line of PN in the $v=0$ state (CDMS entry 045511) with $E_{\text {up }}=271 \mathrm{~K}$ and $A_{i j}=1.5 \times 10^{-2} \mathrm{~s}^{-1}$. The observed ALMA Band 9 spectrum convolved with a uniform circular restoring beam of 0 '.5 at the one-beam ( $\sim 70 \mathrm{au})$ offset from source B of IRAS 16293-2422 in the SW direction is in salmon. The turquoise line is the LTE fit for the transition, assuming a source size that is equal to the beam size of $0^{\prime \prime} .5$, FWHM of $1 \mathrm{~km} \mathrm{~s}^{-1}, T_{\mathrm{ex}}=125 \mathrm{~K}$, and $N=2.1 \times 10^{13} \mathrm{~cm}^{-2} \cdot v_{\mathrm{LSR}}=$ $2.7 \mathrm{~km} \mathrm{~s}^{-1}$ is assumed at this position.

circular restoring beam of 0.5 , yields $N(\mathrm{PN})=2.1 \times 10^{13} \mathrm{~cm}^{-2}$ at the one-beam $(\sim 70 \mathrm{au})$ offset from source B of IRAS 16293-2422 in the $\mathrm{SW}$ direction upon assuming $T_{\mathrm{ex}}=125 \mathrm{~K}$. The observed $J=5-4$ line of PN should not be blended with any other species, based on checks with currently available spectroscopic catalogues. The secondary minor emission bump to the right of the line may stem from two weak acetone lines. The observed frequency shift of about two spectral channels may be due to a velocity shift with respect to the assumed source velocity, which may point towards PN emission tracing a different, adjacent component of the system. Upcoming new dedicated ALMA observations will secure the detection and firmly constrain the column density, excitation temperature, and associated velocity (project-id: 2018.1.01496, PI: Víctor M. Rivilla).

The PILS data in Bands 7 and 3 cover two lines of $\mathrm{P}^{15} \mathrm{~N}$ ( 358.686 and $89.685 \mathrm{GHz}$, with the Band 7 line being also covered in the continuum window of project-id: 2017.1.00568, PI: Lars Kristensen). Two more lines are covered in Bands 7 and 8 of projectid: 2013.1.00061, PI: Audrey Coutens. No emission is seen from $\mathrm{P}^{15} \mathrm{~N}$ in any of these four lines, although synthetic spectra predict these to be very weak ( $<2 \mathrm{mJy}_{\text {beam }}^{-1}$, assuming LTE, a source size that is equal to the beam size of 0.5 , FWHM of $1 \mathrm{~km} \mathrm{~s}^{-1}, T_{\mathrm{ex}}=$ $125 \mathrm{~K}$ and $N=1.5 \times 10^{11} \mathrm{~cm}^{-2}$ ).

\section{A4 PO}

Two weak $\left(A_{i j}=4.7 \times 10^{-6} \mathrm{~s}^{-1}\right)$ lines of $\mathrm{PO}$ are covered by the PILS data in Band 6 with $E_{\text {up }}=37 \mathrm{~K}(239.704$ and $240.268 \mathrm{GHz}$, with the latter line being also covered in project-id: 2016.1.00457, PI: Yoko Oya). Synthetic spectra predict these two lines to be of equal strength; however, in the observations one is completely absent (Fig. A3). This makes it rather unlikely that the other observed line stems from PO. The observed emission at this frequency likely stems from vinyl cyanide and $\mathrm{CH}_{2} \mathrm{DOH}$. Furthermore, synthetic spectra predict these to be very weak $\left(<2 \mathrm{mJy}\right.$ beam $\left.^{-1}\right)$, assuming LTE, a source size that is equal to the beam size of 0 '.5, FWHM of $1 \mathrm{~km} \mathrm{~s}^{-1}, T_{\text {ex }}=125 \mathrm{~K}$ and $N=4.4 \times 10^{14} \mathrm{~cm}^{-2}$. More lines of PO are covered by the PILS data in Band 7: six in total; however, giving only three spectrally resolved lines due to the proximity in frequency. Synthetic spectra predict the observed emission to be $\sim 0.4,48$, and 42 mJy beam $^{-1}$, respectively (Fig. A4). The data show a tentative detection at $329.557 \mathrm{GHz}$, which should not be blended with any other species, based on checks with currently available spectroscopic catalogues. Synthetic spectra predict a detectable line at $329.571 \mathrm{GHz}$ as well; however, there is a very strong quadruple dimethyl ether line to the right, which is causing an absorption feature in the observed spectrum. This absorption likely prevents
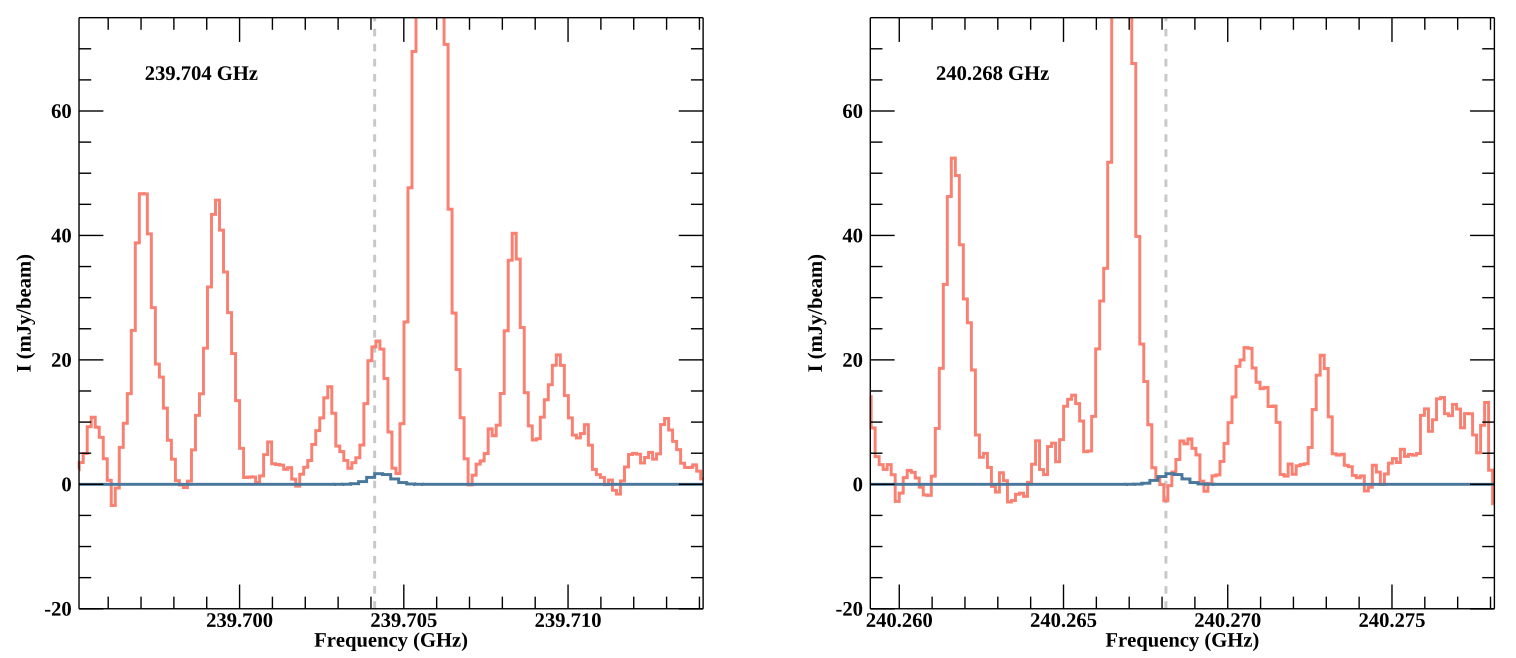

Figure A3. The covered lines of PO (CDMS entry 047507) with $E_{\mathrm{up}}=37 \mathrm{~K}$ and $A_{i j}=4.7 \times 10^{-6} \mathrm{~s}^{-1}$. The observed ALMA Band 6 spectrum convolved with a uniform circular restoring beam of $0 . " 5$ at the one-beam ( $70 \mathrm{au}$ ) offset from source B of IRAS 16293-2422 in the SW direction is in salmon. The turquoise line is the LTE fit for the transition, assuming a source size that is equal to the beam size of 0 '. 5 , FWHM of $1 \mathrm{~km} \mathrm{~s}^{-1}, T_{\mathrm{ex}}=125 \mathrm{~K}$, and $N=4.4 \times 10^{14} \mathrm{~cm}^{-2}$. $v_{\mathrm{LSR}}=2.7 \mathrm{~km} \mathrm{~s}^{-1}$ is assumed at this position. 


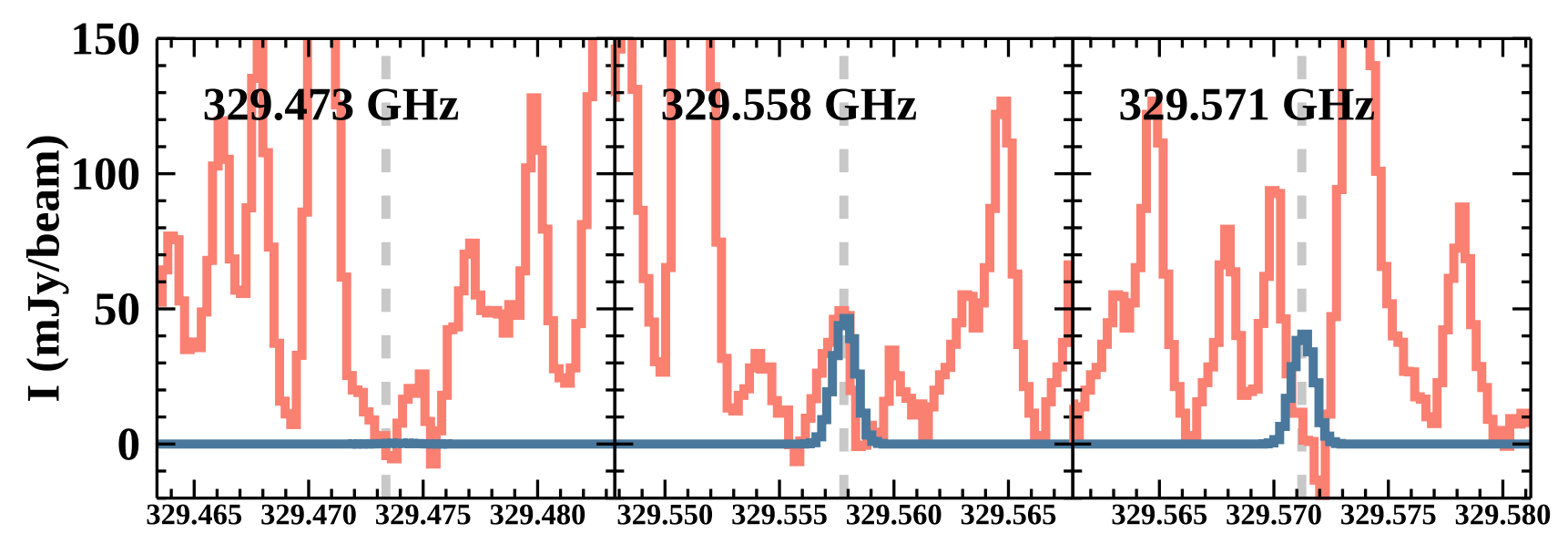

\section{Frequency (GHz)}

Figure A4. The covered lines of PO (CDMS entry 047507) with $E_{\mathrm{up}}=387 \mathrm{~K}$ and $A_{i j}=6.3 \times 10^{-6}$ and $6.6 \times 10^{-4} \mathrm{~s}^{-1}$ for the left-hand and middle jointly with right-hand panels, respectively. The observed ALMA Band 7 spectrum convolved with a uniform circular restoring beam of $0^{\prime \prime} .5$ at the one-beam $(\sim 70$ au) offset from source B of IRAS 16293-2422 in the SW direction is in salmon. The turquoise line is the LTE fit for the transition, assuming a source size that is equal to the beam size of 0 '. 5 , FWHM of $1 \mathrm{~km} \mathrm{~s}^{-1}, T_{\mathrm{ex}}=125 \mathrm{~K}$, and $N=4.4 \times 10^{14} \mathrm{~cm}^{-2} \cdot v_{\mathrm{LSR}}=2.7 \mathrm{~km} \mathrm{~s}^{-1}$ is assumed at this position. The middle panel shows a tentative detection, while the left-hand panel contains a non-detected line due to intrinsic line weakness and the right-hand panel contains a non-detection due to interference with the absorption feature caused by a strong quadrupole line of dimethyl ether to the right.

Table A1. Estimated upper limits on glycine column densities towards IRAS $16293-2422 \mathrm{~B}^{a}$.

\begin{tabular}{lcc}
\hline Glycine & $\mathrm{N}\left(\mathrm{cm}^{-2}\right)$ for $T_{\mathrm{ex}}=125 \mathrm{~K}$ & $\mathrm{~N}\left(\mathrm{~cm}^{-2}\right)$ for $T_{\mathrm{ex}}=300 \mathrm{~K}$ \\
\hline Conformer I & $3.0 \times 10^{15}$ & $8.9 \times 10^{14}$ \\
Conformer II & $1.3 \times 10^{14}$ & $2.7 \times 10^{13}$ \\
\hline
\end{tabular}

Note: ${ }^{a}$ The estimates are obtained under the assumptions of LTE, a source size that is equal to the beam size of 0.5 , FWHM of $1 \mathrm{~km} \mathrm{~s}^{-1}$, and two different assumed values for $T_{\mathrm{ex}}$. Vibrational correction factors and the correction factor for the coupling of line emission with the emission from dense dust at $T_{\mathrm{bg}}$ have been accounted for, as described in the text.

the PO emission from being seen at this frequency. Consequently, only an upper limit is derived for the PO column density of $4.4 \times 10^{14} \mathrm{~cm}^{-2}$ at the one-beam $(\sim 70 \mathrm{au})$ offset from source B of IRAS 16293-2422 in the SW direction.

Five more very weak PO lines have been covered by ALMA observations of IRAS 16293-2422; however, synthetic spectra predict all these to be below $\sim 0.1 \mathrm{mJy}$ beam $^{-1}$ in intensity and consequently, unobservable. Specifically, there are two lines at $\sim 241.574 \mathrm{GHz}$ in Band 6 (project-id: 2013.1.00061, PI: Audrey Coutens); one line at $\sim 349.792 \mathrm{GHz}$ in Band 7 (covered by the PILS survey and by project-id: 2013.1.00018, PI: Markus Schmalzl); ${ }^{2}$ and a double line at $\sim 109.830 \mathrm{GHz}$ (project-id: 2017.1.01247, PI: Giovanni Dipierro). Finally, ten lines of PO lie in the continuum window of project-id: 2016.1.01468, PI: Victor Magalhães, but the strongest of which is predicted to be only $\sim 4$ MJybeam $^{-1}$. As for PN, upcoming new dedicated ALMA observations will

\footnotetext{
${ }^{2}$ Note that this line is only available through the Jet Propulsion Laboratory (JPL) catalogue (Pickett et al. 1998; entry 47006) due to its intrinsic weakness and the intensity cut-off used by the CDMS catalogue.
}

secure the detection and firmly constrain the column density and excitation temperature (project-id: 2018.1.01496, PI: Víctor M. Rivilla).

\section{A5 Glycine}

Spectroscopic characterization of glycine is associated with many large uncertainties. Uncertainties in the ALMA Band 7 frequency range are thought to be at least $\sim 0.15-0.25 \mathrm{MHz}$ for the strongest lines. The molecule has many low-lying modes, which increase the vibrational correction factor, especially at $T_{\mathrm{ex}} \sim 300 \mathrm{~K}$. Gasphase measurements are currently unavailable, only theoretical calculations can be found in the literature. Crude estimates for the vibrational correction factors for Conformer I are 2.05 and 10.65 at 125 and $300 \mathrm{~K}$, respectively. For example, the consideration of an additional low-lying conformer at $1.4 \pm 0.4 \mathrm{kcal} \mathrm{mol}^{-1}$ would introduce an additional correction factor of $1.1-1.5$ at $T_{\mathrm{ex}} \sim 300 \mathrm{~K}$ (H. Müller, private communication).

The PILS Band 7 frequency range covers 949 lines of Conformer I and 1085 lines of Conformer II when making use of the CDMS entries 075511 and 075512 , respectively. These have been used to estimate upper limits on the column density of glycine for excitation temperatures of 125 and $300 \mathrm{~K}$. The obtained values are tabulated in Table A1 upon applying the vibrational correction factors at these two temperatures as derived for Conformer I to both conformers. The synthetic spectra overlaid on to observed spectra around 12 of the strongest predicted lines of each conformer in the PILS frequency range are shown in Figs A5-A8. The column density of glycine used in Table 1 is the sum of the two conformers at $T_{\mathrm{ex}}=300 \mathrm{~K}$, as glycine is expected to be co-spatial with and under comparable excitation conditions as other large complex organic molecules such as glycolaldehyde, ethylene glycol, and formamide. 


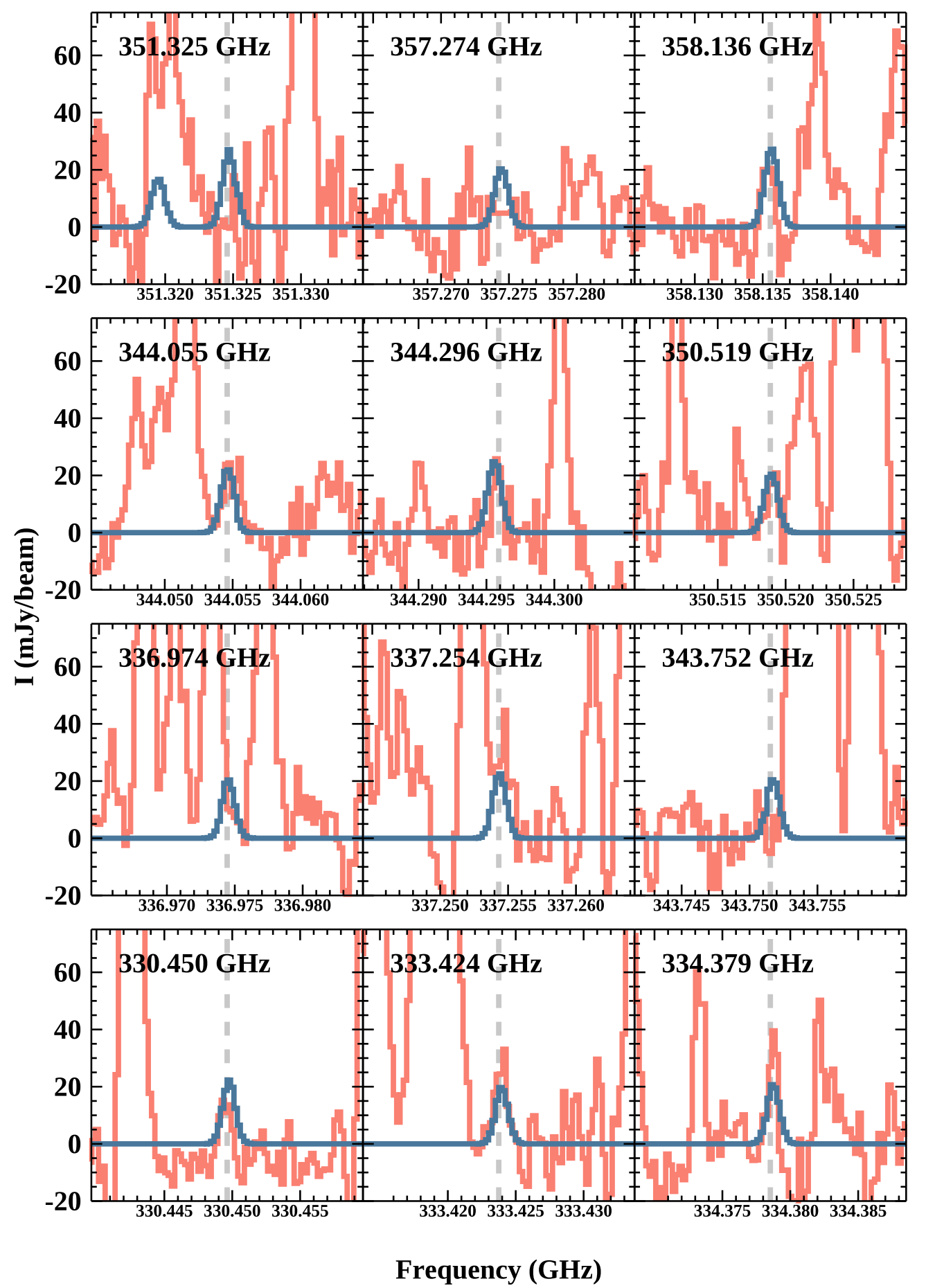

Figure A5. The synthetic spectra overlaid on to observed spectra around 12 of the strongest predicted lines of glycine Conformer I (CDMS entry 075511$)$ in the PILS frequency range. The observed PILS ALMA Band 7 spectrum convolved with a uniform circular restoring beam of $0 . .5$ at the one-beam ( $\sim 70$ au) offset from source B of IRAS 16293-2422 in the SW direction is in salmon. The turquoise lines are the LTE fits for the covered transitions, assuming a source size that is equal to the beam size of 0 '. 5 , FWHM of $1 \mathrm{~km} \mathrm{~s}^{-1}, T_{\mathrm{ex}}=125 \mathrm{~K}$, and $N \leq 3.0 \times 10^{15} \mathrm{~cm}^{-2}$ (upon accounting for the vibrational correction factor and the correction factor for the coupling of line emission with the emission from dense dust at $T_{\mathrm{bg}}$ ). $v_{\mathrm{LSR}}=2.7 \mathrm{~km} \mathrm{~s}^{-1}$ is assumed at this position. 

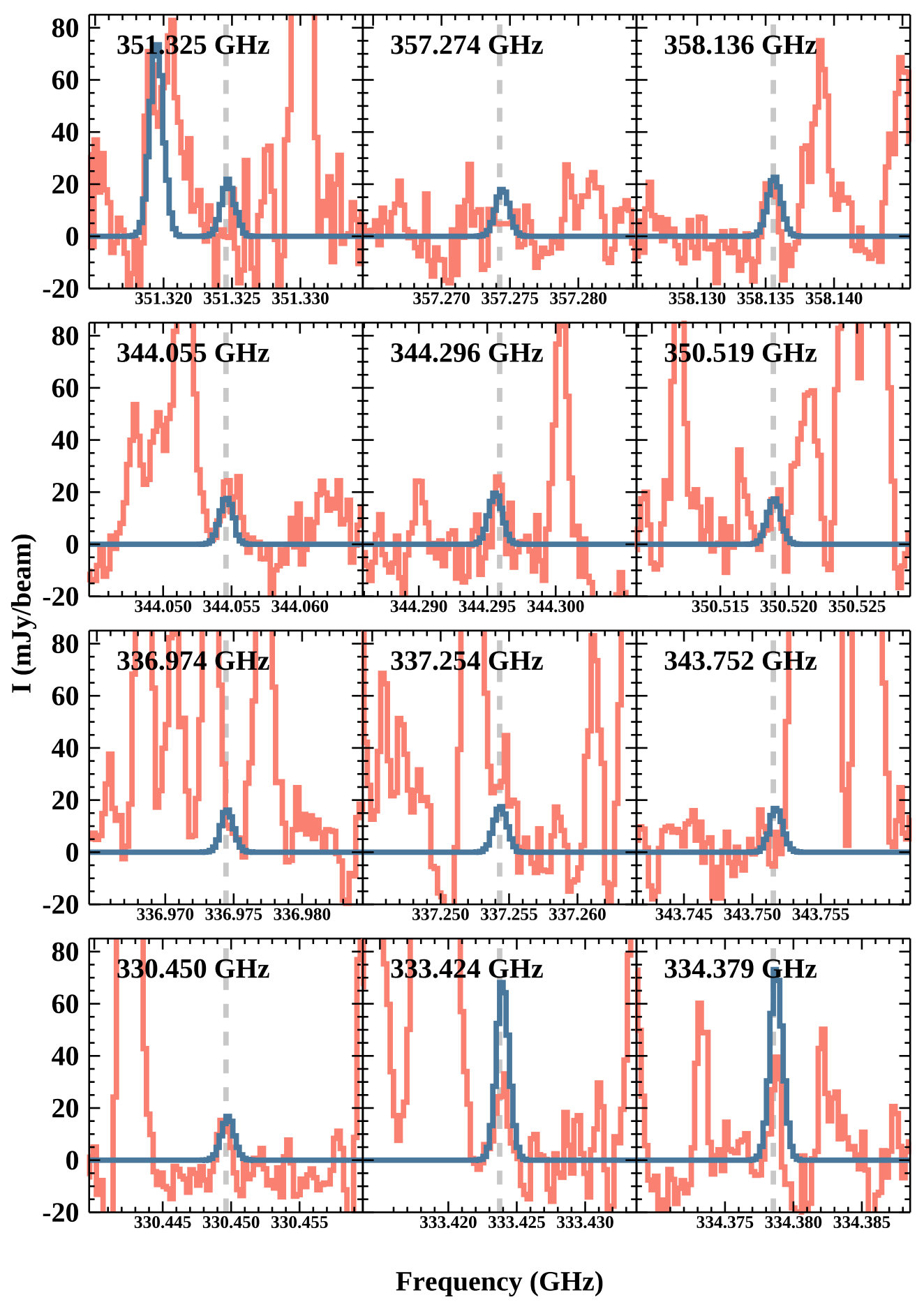

Figure A6. The synthetic spectra overlaid on to observed spectra around 12 of the strongest predicted lines of glycine Conformer I (CDMS entry 075511$)$ in the PILS frequency range. The observed PILS ALMA Band 7 spectrum convolved with a uniform circular restoring beam of 0 .' 5 at the one-beam $(\sim 70$ au) offset from source B of IRAS 16293-2422 in the SW direction is in salmon. The turquoise lines are the LTE fits for the covered transitions, assuming a source size that is equal to the beam size of 0 '. 5 , FWHM of $1 \mathrm{~km} \mathrm{~s}^{-1}, T_{\mathrm{ex}}=300 \mathrm{~K}$, and $N \leq 1.3 \times 10^{14} \mathrm{~cm}^{-2}$ (upon accounting for the vibrational correction factor and the correction factor for the coupling of line emission with the emission from dense dust at $T_{\mathrm{bg}}$ ). $v_{\mathrm{LSR}}=2.7 \mathrm{~km} \mathrm{~s}^{-1}$ is assumed at this position. 


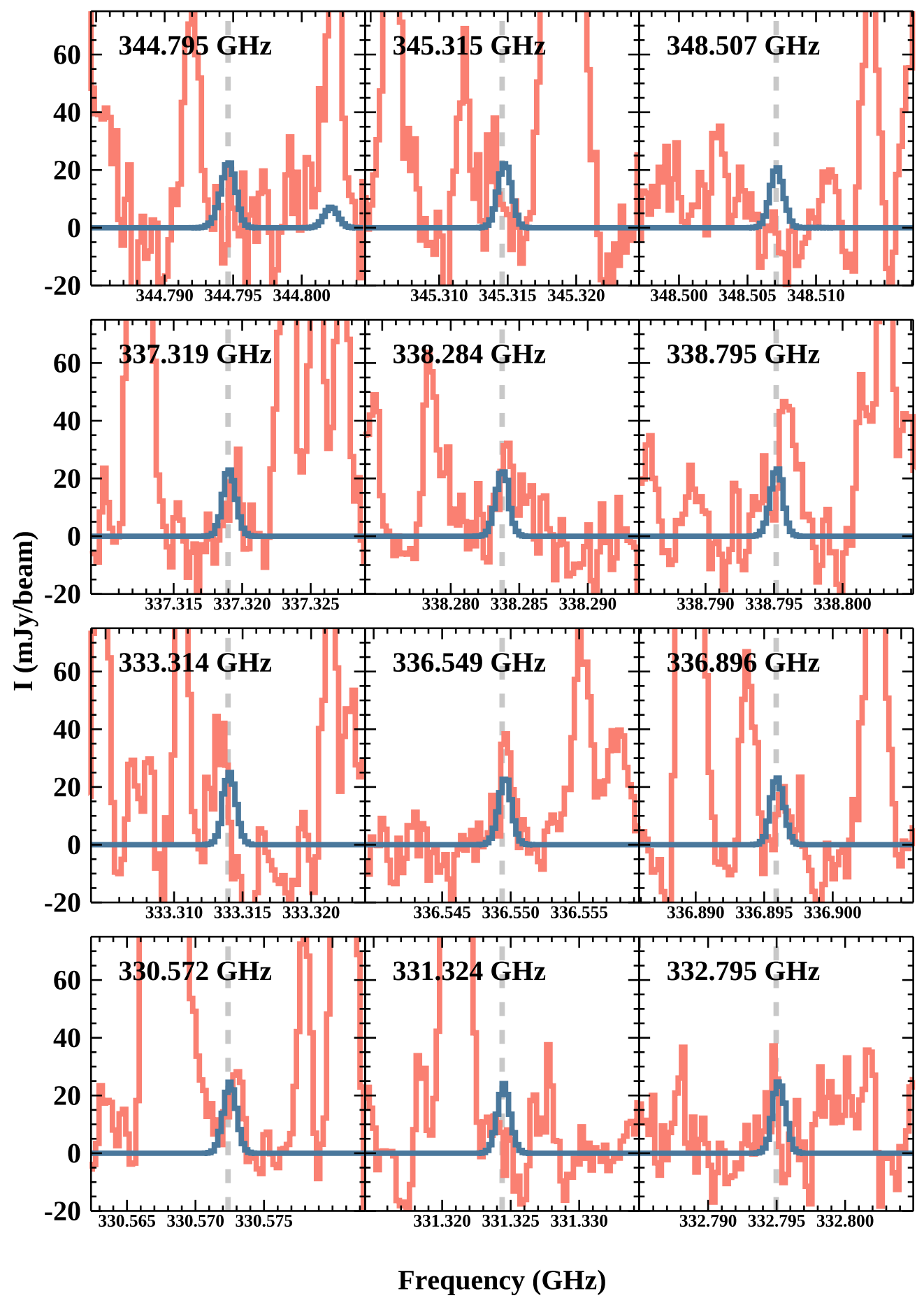

Figure A7. The synthetic spectra overlaid on to observed spectra around 12 of the strongest predicted lines of glycine Conformer II (CDMS entry 075512) in the PILS frequency range. The observed PILS ALMA Band 7 spectrum convolved with a uniform circular restoring beam of $00^{\prime \prime} 5$ at the one-beam ( $~ 70$ au) offset from source B of IRAS 16293-2422 in the SW direction is in salmon. The turquoise lines are the LTE fits for the covered transitions, assuming a source size that is equal to the beam size of 0 '. 5 , FWHM of $1 \mathrm{~km} \mathrm{~s}^{-1}, T_{\mathrm{ex}}=125 \mathrm{~K}$, and $N \leq 8.9 \times 10^{14} \mathrm{~cm}^{-2}$ (upon accounting for the vibrational correction factor and the correction factor for the coupling of line emission with the emission from dense dust at $T_{\mathrm{bg}}$ ). $v_{\mathrm{LSR}}=2.7 \mathrm{~km} \mathrm{~s}^{-1}$ is assumed at this position. 

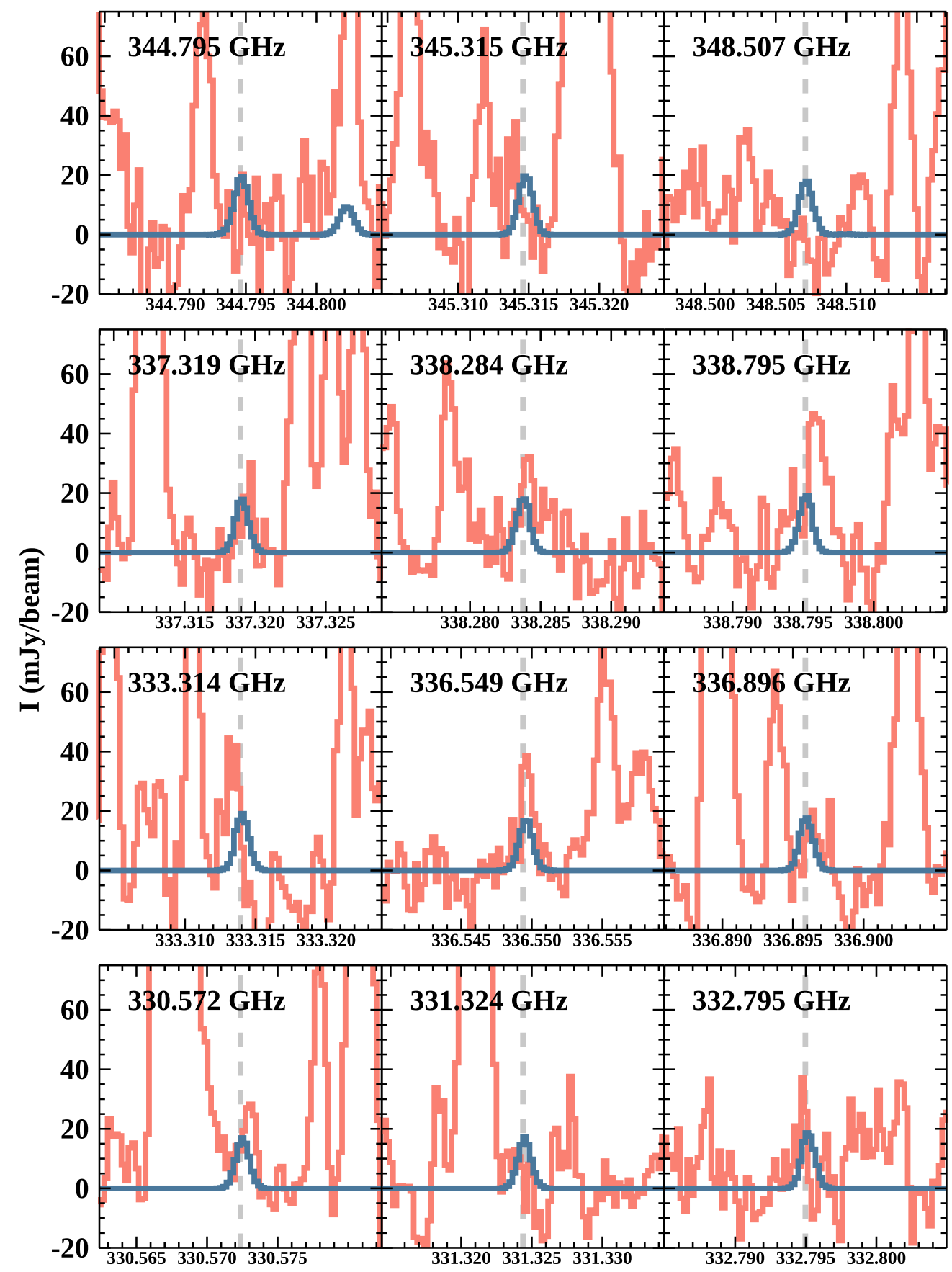

\section{Frequency (GHz)}

Figure A8. The synthetic spectra overlaid on to observed spectra around 12 of the strongest predicted lines of glycine Conformer II (CDMS entry 075512$)$ in the PILS frequency range. The observed PILS ALMA Band 7 spectrum convolved with a uniform circular restoring beam of $0 . ' 5$ at the one-beam ( $\sim 70$ au) offset from source B of IRAS 16293-2422 in the SW direction is in salmon. The turquoise lines are the LTE fits for the covered transitions, assuming a source size that is equal to the beam size of 0.5 , FWHM of $1 \mathrm{~km} \mathrm{~s}^{-1}, T_{\mathrm{ex}}=300 \mathrm{~K}$, and $N \leq 2.7 \times 10^{13} \mathrm{~cm}^{-2}$ (upon accounting for the vibrational correction factor and the correction factor for the coupling of line emission with the emission from dense dust at $T_{\mathrm{bg}}$ ). $v_{\mathrm{LSR}}=2.7 \mathrm{~km} \mathrm{~s}^{-1}$ is assumed at this position. 


\section{APPENDIX B: ADDITIONAL MERGED CORRELATION PLOTS}

This appendix contains three additional correlation plots for the CHO-, N-, S-, P-, and Cl-bearing chemical families. One containing the data of Figs 1-4 merged on to the same scales, while preserving the different respective reference species. The other two figures

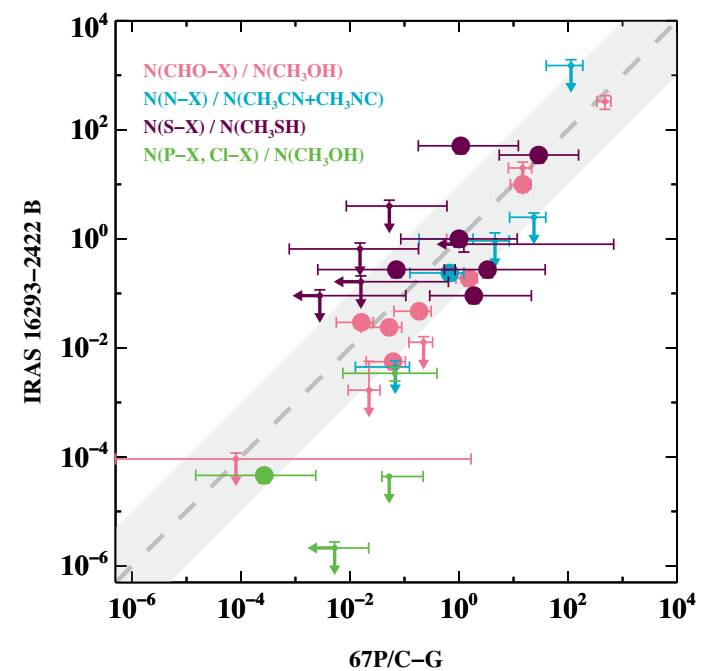

Figure B1. The abundance of CHO-, N-, S-, P-, and Cl-bearing molecules relative to methanol, methyl cyanide, and methyl mercaptan (depending on the family and as indicated in the legend) detected towards the one-beam offset position from IRAS 16293-2422 B versus that measured in 67P/C-G. Each chemical family is marked with a unique colour. The shaded region corresponds to an order of magnitude scatter about the one-to-one linear correlation. Data points that are smaller in size indicate that this pertains to an upper limit or an estimate of sorts either for the protostar, or the comet, or both.

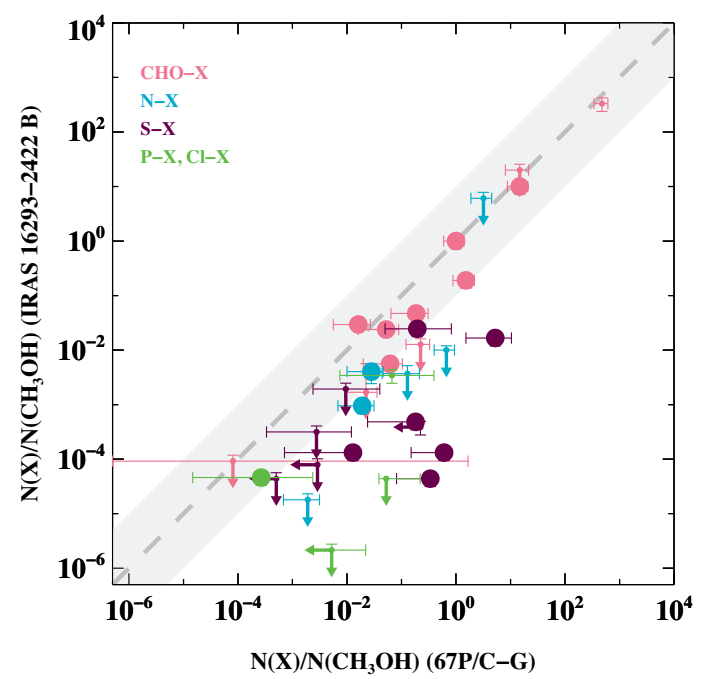

Figure B2. The abundance of CHO-, N-, S-, P-, and Cl-bearing molecules relative to methanol detected towards the one-beam offset position from IRAS $16293-2422$ B versus that measured in $67 \mathrm{P} / \mathrm{C}-\mathrm{G}$. Each chemical family is marked with a unique colour. The shaded region corresponds to an order of magnitude scatter about the one-to-one linear correlation. Data points that are smaller in size indicate that this pertains to an upper limit or an estimate of sorts either for the protostar, or the comet, or both.

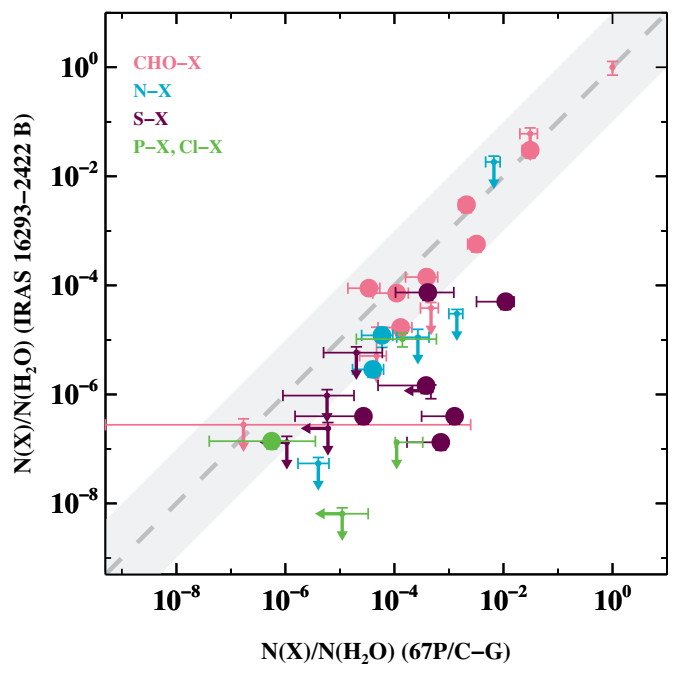

Figure B3. The abundance of CHO-, N-, S-, P-, and Cl-bearing molecules relative to water detected towards the one-beam offset position from IRAS $16293-2422 \mathrm{~B}$ versus that measured in 67P/C-G. Each chemical family is marked with a unique colour. The shaded region corresponds to an order of magnitude scatter about the one-to-one linear correlation. Data points that are smaller in size indicate that this pertains to an upper limit or an estimate of sorts either for the protostar, or the comet, or both.

contain the data when using a common reference species for all molecules probed, namely $\mathrm{CH}_{3} \mathrm{OH}$ and $\mathrm{H}_{2} \mathrm{O}$. Note that the column density of water towards IRAS 16293-2422 B is an estimate based on the value derived for source A (see Table 1 for details).

\section{APPENDIX C: MOLECULES OF IRAS 16293-2422}

In this appendix, the molecules that have been observed towards IRAS 16293-2422 are inventorized. The scales that the respective observations have been carried out on are also considered, and hence, the physical structures that the various species may be tracing are discussed. The cloud scales are $\sim 40 \operatorname{arcsec}-1$ arcmin, the clump scales are $\sim 20-40$ arcsec, the circumbinary envelope scales are $\sim 3-20$ arcsec, the individual envelope scales are $\sim 1-3$ arcsec and the disc scales are $\lesssim 1$ arcsec. Although, these ranges are somewhat arbitrary as it depends on which dust and gas components are being examined. Table 1 summarizes the values used for the plots presented in Section 3.

\section{C1 CHO-bearing molecules}

\section{C1.1 Water $\left(\mathrm{H}_{2} \mathrm{O}\right), \mathrm{m}=18$}

The presence of water has long been established for IRAS 162932422. It was first observed thanks to masers (Wilking \& Claussen 1987), which were quickly realized to be associated with source A (Wootten 1989; Alves et al. 2012) and to also be rapidly variable (Imai, Iwata \& Miyoshi 1999; Colom et al. 2016). $\mathrm{H}_{2} \mathrm{O}$ and HDO emission has since been detected at radio frequencies on envelope scales (van Dishoeck et al. 1995; Stark et al. 2004; Caux et al. 2011; Lis et al. 2016), and has been claimed to originate from both sources, A and B (Chandler et al. 2005; Parise et al. 2005; Jørgensen et al. 2011). Para- $\mathrm{D}_{2} \mathrm{O}$ and ortho- $\mathrm{D}_{2} \mathrm{O}$ have been discovered towards the binary (Butner et al. 2007; Vastel et al. 
2010). $\mathrm{H}_{2} \mathrm{O}$ has been detected in the infrared with ISO/LWS and the Odin satellite (Ceccarelli et al. 1998b; Hjalmarson et al. 2003). $\mathrm{H}_{2} \mathrm{O}$, $\mathrm{HDO}$, and ortho- $\mathrm{D}_{2} \mathrm{O}$ lines were also detected on envelope scales in the infrared with the Herschel Space Observatory (Herschel)/HIFI guaranteed time CHESS key program (Ceccarelli et al. 2010), and para- $\mathrm{D}_{2} \mathrm{O}$ tentatively (Coutens et al. 2013). HDO has additionally been observed with SOFIA (Parise et al. 2012). Combined studies of data from the CHESS program, IRAM $30 \mathrm{~m}$, and JCMT have detected water in the form of $\mathrm{HDO}, \mathrm{p}-\mathrm{H}_{2}^{18} \mathrm{O}, \mathrm{o}-\mathrm{H}_{2}^{18} \mathrm{O}$ and lone lines of $\mathrm{p}-\mathrm{H}_{2}^{17} \mathrm{O}$, o- $\mathrm{H}_{2}^{17} \mathrm{O}$, and $\mathrm{HD}^{18} \mathrm{O}$, but without the ability to spatially resolve the binary (smallest beam size probed was 10'.4; Coutens et al. 2012).

Studies of water on the smallest disc scales have so far been difficult. Persson et al. (2013) detected HDO and $\mathrm{H}_{2}^{18} \mathrm{O}$ on scales of $\sim 0$ '.2 $-55^{\prime \prime} 0$, thereby finally unambiguously disentangling the emission from sources A and B. Towards source A, the lines appear in emission. With the smallest beam size available, the emitting region seems to be marginally spatially resolved. This source size ( 0 '.2 specifically) is then used to derive the column densities while accounting for the beam dilution with bigger beam sizes. There is little dependence on $T_{\mathrm{ex}}$ in the 80 to $300 \mathrm{~K}$ range and the best estimate is $124 \pm 12 \mathrm{~K}$. The values of Persson et al. (2013) used in this paper are given in Table 1. Towards source B, only one line is detected in absorption and column density estimates are not available. However, future work will obtain these column densities (A. Coutens private communication, which will also include a check on the values derived for source A).

\section{C1.2 Carbon monoxide $(\mathrm{CO}), \mathrm{m}=28$}

After the initial identification of IRAS 16293-2422 in the infrared, the first molecules that the region was studied in were ${ }^{12} \mathrm{CO},{ }^{13} \mathrm{CO}$, $\mathrm{C}^{18} \mathrm{O}, \mathrm{C}^{17} \mathrm{O}$ at radio wavelengths (Mundy, Wilking \& Myers 1986; Young, Lada \& Wilking 1986; Menten et al. 1987; Wootten \& Loren 1987; Mundy et al. 1990). Detections in the infrared have also been made with the Infrared Space Observatory (ISO)/Long Wavelength Spectrometer (LWS; Ceccarelli et al. 1998b) and with the Herschel/HIFI guaranteed time CHESS key program (Ceccarelli et al. 2010). Studies were subsequently carried out at available increasing spatial resolutions (Blake et al. 1994; Lis et al. 2002; Yeh et al. 2008; Rao et al. 2009; Caux et al. 2011; Jørgensen et al. 2011); and CO envelope abundances started to be modelled with jump profiles (Schöier et al. 2002, 2004). ${ }^{13} \mathrm{C}^{18} \mathrm{O}$ and ${ }^{13} \mathrm{C}^{17} \mathrm{O}$ have also been detected on envelope scales (Blake et al. 1994). It has been shown that $\mathrm{CO}$ and its isotopologues are present on the large cloud and core scales in L1689N; and on the smaller circumbinary envelope, and disc-A and -B scales. It also traces the outflows from the system (Walker et al. 1988; Mizuno et al. 1990), as also solidified by the detection of $\mathrm{CO}^{+}$in ISO/LWS data (Ceccarelli et al. 1998c, although still remaining to be verified with ALMA observations). Most recently, studies with spatial resolution superseding that of PILS have been used to study the shocks and outflows stemming from source A as traced by carbon monoxide emission (Favre et al. 2014; Girart et al. 2014; Kristensen et al. 2013, based on the line labelled in Baryshev et al. 2015; van der Wiel et al. 2019). Unfortunately, estimates of the column density are not provided in those works as too few lines are targeted at a time. Jacobsen et al. (2018b) developed sophisticated models of the binary IRAS $16293-2422$ system based on ${ }^{13} \mathrm{CO}, \mathrm{C}^{17} \mathrm{O}$, and $\mathrm{C}^{18} \mathrm{O}$ $J=3-2$ observations. The latest derivation of the column density is presented in Appendix A.

\section{C1.3 Molecular oxygen $\left(\mathrm{O}_{2}\right), \mathrm{m}=32$}

Taquet et al. (2018) searched for molecular oxygen towards source $\mathrm{B}$ through the ${ }^{16} \mathrm{O}^{18} \mathrm{O}$ line at $234 \mathrm{GHz}$, claiming at most a tentative detection.

\section{C1.4 Hydroperoxyl $\left(\mathrm{HO}_{2}\right), \mathrm{m}=33$}

Taquet et al. (2018) searched for $\mathrm{HO}_{2}$ towards source $\mathrm{B}$ and derived an upper limit.

\section{C1.5 Hydrogen peroxide $\left(\mathrm{H}_{2} \mathrm{O}_{2}\right), \mathrm{m}=34$}

Taquet et al. (2018) searched for $\mathrm{H}_{2} \mathrm{O}_{2}$ towards source $\mathrm{B}$, but were unable to derive an upper limit due to blending with other species.

\section{C1.6 Formaldehyde $\left(\mathrm{H}_{2} \mathrm{CO}\right), \mathrm{m}=30$}

$\mathrm{H}_{2} \mathrm{CO}, \mathrm{H}_{2}^{13} \mathrm{CO}$, HDCO, $\mathrm{H}_{2} \mathrm{C}^{18} \mathrm{O}$, and $\mathrm{D}_{2} \mathrm{CO}$ have been detected on envelope scales, and with both ortho- and para- versions showing self-absorption (van Dishoeck et al. 1995; Ceccarelli et al. 1998a; Loinard et al. 2000; Chandler et al. 2005; Caux et al. 2011; van der Wiel et al. 2019) and towards both protostars, even as $\mathrm{D}_{2}^{13} \mathrm{CO}$ (Jørgensen et al. 2011). A number of models have addressed the abundance of formaldehyde and its release via thermal desorption in the vicinity of the protostars (Ceccarelli et al. 2000c, 2001; Schöier et al. 2002; Ceccarelli et al. 2003; Schöier et al. 2004). $\mathrm{H}_{2} \mathrm{CO}$ was detected on envelope scales in the infrared with the Herschel/HIFI guaranteed time CHESS key program (Ceccarelli et al. 2010). Formaldehyde has also been shown to be associated with larger scale shocked regions (Castets et al. 2001), although it is significantly more abundant in the warm inner regions around the protostars (Lindberg et al. 2017). Individual lines of $\mathrm{D}_{2} \mathrm{CO}$ and HDCO have been labelled in high spatial resolution observations of Baryshev et al. (2015) and Martín-Doménech et al. (2016). Estimates of the column densities towards A and B have been provided in Rivilla et al. (2019); however, corrections for optically thick emission have not been carried out. Persson et al. (2018) identified $\mathrm{H}_{2} \mathrm{CO}, \mathrm{H}_{2}^{13} \mathrm{CO}$, $\mathrm{H}_{2} \mathrm{C}^{17} \mathrm{O}, \mathrm{H}_{2} \mathrm{C}^{18} \mathrm{O}, \mathrm{HDCO}, \mathrm{HDC}^{18} \mathrm{O}, \mathrm{D}_{2} \mathrm{CO}, \mathrm{D}_{2}^{13} \mathrm{CO}$ towards source $\mathrm{B}$ and derived accurate column densities. Manigand et al. submitted identified $\mathrm{H}_{2} \mathrm{CO}, \mathrm{H}_{2}^{13} \mathrm{CO}, \mathrm{H}_{2} \mathrm{C}^{17} \mathrm{O}, \mathrm{H}_{2} \mathrm{C}^{18} \mathrm{O}, \mathrm{HDCO}$, and $\mathrm{D}_{2} \mathrm{CO}$ towards source $\mathrm{A}$ and derived accurate column densities.

\section{C1.7 Methanol $\left(\mathrm{CH}_{3} \mathrm{OH}\right), \mathrm{m}=32$}

$\mathrm{CH}_{3} \mathrm{OH},{ }^{13} \mathrm{CH}_{3} \mathrm{OH}, \mathrm{CH}_{2} \mathrm{DOH}, \mathrm{CHD}_{2} \mathrm{OH}, \mathrm{CH}_{3} \mathrm{OD}$, and $\mathrm{CD}_{3} \mathrm{OH}$ have been detected on envelope scales (van Dishoeck et al. 1995; Parise et al. 2002, 2004; Kuan et al. 2004; Chandler et al. 2005; Caux et al. 2011). On larger cloud and core scales, $\mathrm{CH}_{3} \mathrm{OH}$ emission seems to arise from the material entrained by a wide-angle stellar wind stemming from the protostar and is estimated to be enhanced by a factor of $\sim 500$ relative to the ambient gas (Garay et al. 2002; Lis et al. 2016; Lindberg et al. 2017). $\mathrm{CH}_{3} \mathrm{OH}$ was detected on envelope scales in the infrared with the Herschel/HIFI guaranteed time CHESS key program (Ceccarelli et al. 2010). $\mathrm{CH}_{3} \mathrm{OH}$ and ${ }^{13} \mathrm{CH}_{3} \mathrm{OH}$ have been observed towards the two protostars individually (Jørgensen et al. 2011), even at spatial resolution superseding that of PILS - lines of $\mathrm{CH}_{3} \mathrm{OH},{ }^{13} \mathrm{CH}_{3} \mathrm{OH}, \mathrm{CH}_{2} \mathrm{DOH}$ have been labelled in Baryshev et al. (2015) and Martín-Doménech et al. (2016). Towards source $\mathrm{B}, \mathrm{CH}_{3} \mathrm{OH}$ emission displays inverse $\mathrm{P}$ Cygni profiles, indicating infall (Zapata et al. 2013). Estimates of 
the column densities towards A and B have been provided in Rivilla et al. (2019); however, corrections for optically thick emission have not been carried out. Additionally, $\mathrm{CH}_{3} \mathrm{OH}$ has been argued to display abundance enhancements at the centrifugal barrier in both sources (Oya et al. 2016, 2018); however, these studies claim optically thin emission, which is likely not the case as analysed in Jørgensen et al. $(2016,2018)$ based on $\mathrm{CH}_{3} \mathrm{OH}, \mathrm{CH}_{2} \mathrm{DOH}$, and $\mathrm{CH}_{3} \mathrm{OD}$ emission and accurate column densities towards source $\mathrm{B}$. $\mathrm{CH}_{3}^{18} \mathrm{OH}, \mathrm{CH}_{2} \mathrm{DOH}$, and $\mathrm{CH}_{3} \mathrm{OD}$ have also been detected towards source A, like towards source $\mathrm{B}$, and accurate column densities have been derived (Manigand et al. submitted).

\section{C1.8 Ethanol $\left(\mathrm{C}_{2} \mathrm{H}_{5} \mathrm{OH}\right), \mathrm{m}=46$}

g- $\mathrm{C}_{2} \mathrm{H}_{5} \mathrm{OH}$ was initially detected towards source A (Huang et al. 2005) and then towards both sources (Bisschop et al. 2008; Jørgensen et al. 2011). Jørgensen et al. (2018) identified $\mathrm{CH}_{3} \mathrm{CH}_{2} \mathrm{OH}$, a-a- $\mathrm{CH}_{2} \mathrm{DCH}_{2} \mathrm{OH}$, a-s- $\mathrm{CH}_{2} \mathrm{DCH}_{2} \mathrm{OH}$, a$\mathrm{CH}_{3} \mathrm{CHDOH}$, a- $\mathrm{CH}_{3} \mathrm{CH}_{2} \mathrm{OD}$, a- $\mathrm{CH}_{3}^{13} \mathrm{CH} 2 \mathrm{OH}, \mathrm{a}^{13} \mathrm{CH}_{3} \mathrm{CH}_{2} \mathrm{OH}$ towards source $\mathrm{B}$ and derived accurate column densities. Manigand et al. submitted identified $\mathrm{CH}_{3} \mathrm{CH}_{2} \mathrm{OH}$, a-a- $\mathrm{CH}_{2} \mathrm{DCH}_{2} \mathrm{OH}$, a-s$\mathrm{CH}_{2} \mathrm{DCH}_{2} \mathrm{OH}$, a- $\mathrm{CH}_{3} \mathrm{CHDOH}$, a- $\mathrm{CH}_{3} \mathrm{CH}_{2} \mathrm{OD}$ towards source A and derived accurate column densities.

\section{C1.9 Ketene/Ethenone $\left(\mathrm{CH}_{2} \mathrm{CO}\right), \mathrm{m}=42$}

$\mathrm{CH}_{2} \mathrm{CO}$ has been detected on envelope scales (van Dishoeck et al. 1995; Caux et al. 2011) and towards the two sources individually (Kuan et al. 2004; Bisschop et al. 2008; Jørgensen et al. 2011). Inverse P Cygni profiles are seen near source B, unlike near source A, suggesting on-going infall and face-on orientation (Pineda et al. 2012). Lines of $\mathrm{CH}_{2} \mathrm{CO}$ are also labelled in Majumdar et al. (2016). Jørgensen et al. (2018) identified $\mathrm{CH}_{2} \mathrm{CO},{ }^{13} \mathrm{CH}_{2} \mathrm{CO}, \mathrm{CH}_{2}^{13} \mathrm{CO}$, CHDCO towards source $\mathrm{B}$ and derived accurate column densities. Manigand et al. submitted identified $\mathrm{CH}_{2} \mathrm{CO}$ and $\mathrm{CHDCO}$ towards source $\mathrm{A}$ and derived accurate column densities.

\section{C1.10 Formic acid ( $\mathrm{HCOOH}), \mathrm{m}=46$}

$\mathrm{HCOOH}$ has been detected towards source B (Cazaux et al. 2003; Remijan \& Hollis 2006) and towards A (Jørgensen et al. 2011), as has HCOOD (Jørgensen et al. 2011). Jørgensen et al. (2018) and Manigand et al. submitted identified $\mathrm{t}-\mathrm{HCOOH}, \mathrm{t}-\mathrm{H}^{13} \mathrm{COOH}, \mathrm{t}-$ $\mathrm{DCOOH}, \mathrm{t}-\mathrm{HCOOD}$ towards sources $\mathrm{B}$ and $\mathrm{A}$, and derived accurate column densities.

\section{C1.11 Acetaldehyde $\left(\mathrm{CH}_{3} \mathrm{CHO}\right), \mathrm{m}=44$}

$\mathrm{CH}_{3} \mathrm{CHO}$ was detected in its - $\mathrm{A}$ and -E forms towards both sources (Cazaux et al. 2003; Bisschop et al. 2008; Jørgensen et al. 2011) and also on envelope scales (Caux et al. 2011). Its lines have also been labelled in Baryshev et al. (2015) and Martín-Doménech et al. (2016), and mentioned in Girart et al. (2014). Lykke et al. (2017); Jørgensen et al. (2018); Coudert et al. (2019) identified $\mathrm{CH}_{3} \mathrm{CHO}$, $\mathrm{CH}_{3} \mathrm{CDO},{ }^{13} \mathrm{CH}_{3} \mathrm{CHO}, \mathrm{CH}_{3}^{13} \mathrm{CHO}$, and $\mathrm{CH}_{2} \mathrm{DCHO}$ towards source $\mathrm{B}$ and derived accurate column densities; and $\mathrm{CH}_{3} \mathrm{CHO}$ towards source A (Manigand et al. submitted).

\section{C1.12 Methyl formate $\left(\mathrm{HCOOCH}_{3}\right), \mathrm{m}=60$}

$\mathrm{HCOOCH}_{3}$ has been detected in its -A and -E forms towards both protostars individually (Cazaux et al. 2003; Bottinelli et al. 2004; Kuan et al. 2004; Chandler et al. 2005; Remijan \& Hollis 2006; Shiao et al. 2010). On circumbinary envelope scales, initially, only upper limits were available (Remijan et al. 2003), but then a detection was also made (Caux et al. 2011). $\mathrm{DCOOCH}_{3}$ was tentatively detected with the TIMASSS survey (Demyk et al. 2010). Inverse P Cygni profiles are seen near source B, unlike near source A, suggesting on-going infall and face-on orientation (Pineda et al. 2012; Favre et al. 2014). Its lines have also been labelled in Baryshev et al. (2015) and Martín-Doménech et al. (2016). Additionally, $\mathrm{HCOOCH}_{3}$ has been argued to display abundance enhancements at the centrifugal barrier in both sources (Oya et al. 2016, 2018); however, these studies claim optically thin emission, which is likely not the case as analysed in Jørgensen et al. (2018) based on $\mathrm{CH}_{3} \mathrm{OCHO}, \mathrm{CH}_{3} \mathrm{OCDO}, \mathrm{CH}_{2} \mathrm{DOCHO}$, and $\mathrm{CH}_{3} \mathrm{O}^{13} \mathrm{CHO}$ emission towards source B. Manigand et al. (2019) and Manigand et al. submitted detected $\mathrm{CHD}_{2} \mathrm{OCHO}, \mathrm{CH}_{2} \mathrm{DOCHO}, \mathrm{CH}_{3} \mathrm{OCDO}$, $\mathrm{CH}_{3} \mathrm{O}^{13} \mathrm{CHO}$, and $\mathrm{CH}_{3} \mathrm{OCHO}$ towards both protostars. Accurate column densities were derived in these works (Jørgensen et al. 2018; Manigand et al. 2019; Manigand et al. submitted).

\section{C1.13 Acetic acid $\left(\mathrm{CH}_{3} \mathrm{COOH}\right), \mathrm{m}=60$}

$\mathrm{CH}_{3} \mathrm{COOH}$ was detected in its - $\mathrm{E}$ form towards source B (Cazaux et al. 2003). Subsequently, in its -E and -A forms towards source A, but with only upper limits derived towards source B (Shiao et al. 2010). Higher spatial resolution observations secured its detection towards both sources (Jørgensen et al. 2011). On envelope scales, only upper limits have been derived (Remijan et al. 2003). $\mathrm{CH}_{3} \mathrm{COOH}$ was again observed towards source B by Jørgensen et al. (2016) with an accurate column density being derived, and towards source A (Manigand et al. submitted).

\section{C1.14 Glycolaldehyde $\left(\mathrm{CH}_{2} \mathrm{OHCHO}\right), \mathrm{m}=60$}

The first time detection towards both sources was obtained by Jørgensen et al. (2012). The submitted paper of Zhou et al. (2018) erroneously claims the first time detection of vibrationally excited glycolaldehyde, as $v=1$ and $v=2$ lines have been detected towards both sources already in Jørgensen et al. (2012). Column densities towards A and B have been derived in Rivilla et al. (2019) on $\sim 1$ arcsec scales. Jørgensen et al. (2016) identified $\mathrm{CH}_{2} \mathrm{OHCHO}, \mathrm{CHDOH}-$ $\mathrm{CHO}, \mathrm{CH}_{2} \mathrm{ODCHO}, \mathrm{CH}_{2} \mathrm{OHCDO},{ }^{13} \mathrm{CH}_{2} \mathrm{OHCHO}, \mathrm{CH}_{2} \mathrm{OH}^{13} \mathrm{CHO}$ towards source $\mathrm{B}$ and provided accurate column densities on $\sim 0$.'5 scales; and $\mathrm{CH}_{2} \mathrm{OHCHO}$ towards source A (Manigand et al. submitted).

\section{C1.15 Ethylene glycol/Ethane-1,2-diol $\left(\left(\mathrm{CH}_{2} \mathrm{OH}\right)_{2}\right), \mathrm{m}=62$}

A tentative detection towards source $\mathrm{B}$ has been reported in Jørgensen et al. (2012). Jørgensen et al. (2016) firmly identified aGg'-ethylene glycol and gGg'-ethylene glycol towards source B and derived accurate column densities. The detection of ethylene glycol towards source A has been claimed by the submitted paper of Zhou et al. (2018); however, this was already established earlier by the PILS team (Manigand et al. submitted). 


\section{C1.16 Dimethyl ether/Methoxymethane $\left(\mathrm{CH}_{3} \mathrm{OCH}_{3}\right), \mathrm{m}=46$}

$\mathrm{CH}_{3} \mathrm{OCH}_{3}$ was initially detected towards source B (Cazaux et al. 2003), but then towards both protostars (Chandler et al. 2005; Huang et al. 2005; Jørgensen et al. 2011) and on envelope scales (Caux et al. 2011). $\mathrm{CH}_{2} \mathrm{DOCH}_{3}$ has also been detected on envelope scales (Richard et al. 2013). $\mathrm{CH}_{3} \mathrm{OCH}_{3}$ lines have been labelled in Baryshev et al. (2015) and in Martín-Doménech et al. (2016). Jørgensen et al. (2018) and Manigand et al. submitted identified $\mathrm{CH}_{3} \mathrm{OCH}_{3},{ }^{13} \mathrm{CH}_{3} \mathrm{OCH}_{3}$, asym- $\mathrm{CH}_{2} \mathrm{DOCH}_{3}$, sym- $\mathrm{CH}_{2} \mathrm{DOCH}_{3}$ towards sources $\mathrm{A}$ and $\mathrm{B}$, and derived accurate column densities.

\section{C1.17 Acetone/Propan-2-one $\left(\left(\mathrm{CH}_{3}\right)_{2} \mathrm{CO}\right), \mathrm{m}=58$}

$\left(\mathrm{CH}_{3}\right)_{2} \mathrm{CO}$ has been detected towards both sources by Jørgensen et al. (2011), and once more by Lykke et al. (2017) and Manigand et al. submitted with accurate column densities being derived.

\section{C1.18 Ethylene oxide $\left(c-\mathrm{C}_{2} \mathrm{H}_{4} \mathrm{O}\right), \mathrm{m}=44$}

Ethylen oxide was detected towards source B by Lykke et al. (2017) and an accurate column density was derived; towards source A (Manigand et al. submitted).

\section{C1.19 Propanal/Propionaldehyde $\left(\mathrm{C}_{2} \mathrm{H}_{5} \mathrm{CHO}\right), \mathrm{m}=58$}

Propanal was detected towards sources B and A by Lykke et al. (2017) and Manigand et al. submitted, and accurate column densities were derived.

\section{C1.20 Vinyl alcohol $\left(\mathrm{CH}_{2} \mathrm{CHOH}\right), \mathrm{m}=44$}

An upper limit for vinyl alcohol towards source B was derived by Lykke et al. (2017).

\section{C1.21 Methoxymethanol $\left(\mathrm{CH}_{3} \mathrm{OCH}_{2} \mathrm{OH}\right)$}

Methoxymethanol has been detected towards source B and an accurate column density has been derived, while for source A an upper limit has been computed (Manigand et al. submitted).

\section{C1.22 Trans ethyl methyl ether $\left(t-\mathrm{C}_{2} \mathrm{H}_{5} \mathrm{OCH}_{3}\right)$}

Trans ethyl methyl ether has been detected towards source B and an accurate column density has been derived, while for source A an upper limit has been computed (Manigand et al. submitted).

\section{C1.23 Glycine $\left(\mathrm{NH}_{2} \mathrm{CH}_{2} \mathrm{COOH}\right), \mathrm{m}=75$}

A search for glycine was carried towards IRAS 16293-2422 with IRAM 30-m telescope yielding an upper limit based on the nondetection (Ceccarelli et al. 2000b). The latest derivation of an upper limit on the column density is presented in Appendix A.

\section{C1.24 Butane-1,2-diol $\left(\mathrm{HOCH}_{2}(\mathrm{HO}) \mathrm{CHCH}_{2} \mathrm{CH}_{3}\right), \mathrm{m}=90$}

Six conformers of 1,2-butanediol were searched for towards source B based ALMA data, but only an upper limit of $1 \times 10^{13} \mathrm{~cm}^{-2}$ has been derived (Vigorito et al. 2018).

\section{C1.25 Hydroxide $\left(\mathrm{OH}^{-}\right), \mathrm{m}=17$}

$\mathrm{OH}^{-}$is an anion, which has been detected on circumbinary envelope scales with ISO/LWS (Ceccarelli et al. 1998b), but remains to be conclusively confirmed. OD has also been discovered on these scales with SOFIA (Parise et al. 2012). OH has been observed at $\mathrm{cm}$ wavelengths and its emission appears to have variable flares (Colom et al. 2016).

\section{C1.26 Formyl cation $\left(\mathrm{HCO}^{+}\right), \mathrm{m}=29$}

$\mathrm{HCO}^{+}$cation has been detected on large cloud and core scales, and smaller circumbinary envelope scales (Mizuno et al. 1990; van Dishoeck et al. 1995; Narayanan, Walker \& Buckley 1998; Lis et al. 2002; Stark et al. 2004; Caux et al. 2011; Lis et al. 2016; Quénard et al. 2018a). Its lines show blueshifted self-absorption, which has been interpreted as a sign of expansion (Choi, Panis \& Evans 1999). $\mathrm{DCO}^{+}$has also been detected on these scales (Wootten \& Loren 1987; van Dishoeck et al. 1995; Lis et al. 2002; Stark et al. 2004; Caux et al. 2011; Lindberg et al. 2017; Quénard et al. 2018a), and so have $\mathrm{H}^{13} \mathrm{CO}^{+}$(van Dishoeck et al. 1995; Narayanan et al. 1998; Lis et al. 2002; Stark et al. 2004; Rao et al. 2009; Caux et al. 2011; Jørgensen et al. 2011; Quénard et al. 2018a) and $\mathrm{HC}^{18} \mathrm{O}^{+}$ (Blake et al. 1994; van Dishoeck et al. 1995; Caux et al. 2011; Quénard et al. 2018a). D ${ }^{13} \mathrm{CO}^{+}$(Caux et al. 2011; Quénard et al. 2018a) and $\mathrm{HC}^{17} \mathrm{O}^{+}$have also been detected (Caux et al. 2011). $\mathrm{HCO}^{+}$and $\mathrm{H}^{13} \mathrm{CO}^{+}$in the infrared was detected on envelope scales in the infrared with the Herschel/HIFI guaranteed time CHESS key program (Ceccarelli et al. 2010). On the scale of individual envelopes, $\mathrm{DCO}^{+}$has been detected only towards A (Jørgensen et al. 2011). Most recently, the distribution of $\mathrm{DCO}^{+}$has been analysed by Murillo et al. (2018b) on the scale of the individual protostars and the circumbinary envelope.

\section{C1.27 Formyl radical $(\mathrm{HCO}), \mathrm{m}=29$}

The HCO radical has been detected in the TIMASSS survey and analysed by Bacmann \& Faure (2016). Very recently it has been mapped on 1 arcsec scales towards both protostars, whereby blueshifted absorption is seen towards both sources, implying that A is undergoing infall just like B (Rivilla et al. 2019).

\section{C1.28 Hydrocarboxyl $\left(\mathrm{HOCO}^{+}\right), \mathrm{m}=45$}

A detection of $\mathrm{HOCO}^{+}$has been claimed based on two lines by Majumdar et al. (2018) on envelope scales.

\section{C2 N-bearing molecules}

\section{C2.1 Ammonia $\left(\mathrm{NH}_{3}\right), \mathrm{m}=17$}

$\mathrm{NH}_{3}$ is a symmetric molecule with very weak hyperfine (inversion) emission lines at radio frequencies. It was detected on large scales of the cloud and core in emission (Menten et al. 1987; Wootten \& Loren 1987; Mizuno et al. 1990; Mundy et al. 1990) and in emission and absorption in its ortho- and para- forms with the Herschel/HIFI guaranteed time CHESS key program (Ceccarelli et al. 2010; HilyBlant et al. 2010). $\mathrm{NH}_{2} \mathrm{D}$ was detected on smaller envelope scales (van Dishoeck et al. 1995; Caux et al. 2011). Para-NH2 D and ortho$\mathrm{NH}_{3}$ were also studied across L1689N based on Herschel/HIFI data by Lis et al. (2016). Their data clearly showed strong $\mathrm{NH}_{3}$ emission towards the location of IRAS 16293-2422 suggesting 
$\mathrm{NH}_{3}$ emission to be optically thick towards that position, and also subject to absorption from the foreground material. Para- $\mathrm{NH}_{2} \mathrm{D}$ was not detected towards IRAS 16293-2422 at those frequencies. Unfortunately, the field of view of their ALMA ACA mosaic, which included $\mathrm{a} \mathrm{ND}_{3}$ line, did not cover the position of IRAS 16293-2422.

Subsequently, at the moment, the only estimate of the quantity of ammonia in IRAS 16293-2422 available is that on the scales of its circumbinary envelope. Lis et al. (2016) do not provide an estimate of the column density of $\mathrm{NH}_{3}$ towards the position of IRAS 16293-2422. Caux et al. (2011) do not derive the column density of $\mathrm{NH}_{2} \mathrm{D}$ due to severe blending. Estimates from infrared $\mathrm{NH}_{3}$ observations of Hily-Blant et al. (2010) span a range larger than an order of magnitude for a source size of $12-18$ arcsec. Observations of van Dishoeck et al. (1995) of $\mathrm{NH}_{2} \mathrm{D}$ can also be used to calculate the column density of $\mathrm{NH}_{3}$ within a range spanning an order of magnitude, as the appropriate $\mathrm{D} / \mathrm{H}$ ratio to use is unclear (hence, a range of $0.05-0.005$ is considered in this work; e.g. Persson et al. 2014) for a source size of 20 arcsec. Mundy et al. (1990) provided the only direct estimate of the column density of $\mathrm{NH}_{3}$ for a source size of 20 arcsec; however, this value may be too low due to optical thickness of ammonia emission. In fact, this value gives the lower limit of the range of ammonia column densities used in this work. Finally, the $\mathrm{NH}_{3}$ column density estimate of Mizuno et al. (1990) is for a beam size of 40 arcsec. If a source size of 20 arcsec, representative of the circumbinary envelope, is assumed, then the column density of Mizuno et al. (1990) can be corrected for beam dilution. These four values are then used to obtain the range of ammonia column densities considered in this work (Table 1).

\section{C2.2 Hydrogen cyanide (HCN), $\mathrm{m}=27$}

HCN lines show deep self-absorption (Mizuno et al. 1990), which has been observed to be blueshifted and interpreted as a sign of expansion (Choi et al. 1999). Studies on large cloud and core scales have testified to the presence of $\mathrm{H}^{13} \mathrm{CN}$ and DCN (Lis et al. 2002). Smaller envelope scales have been shown to contain HCN (including its self-absorption), $\mathrm{H}^{13} \mathrm{CN}, \mathrm{HC}^{15} \mathrm{~N}$, DCN originating from the two protostars (van Dishoeck et al. 1995; Kuan et al. 2004; Takakuwa et al. 2007; Caux et al. 2011; Jørgensen et al. 2011; Wampfler et al. 2014; van der Wiel et al. 2019). Its envelope abundances can be reproduced with jump profiles (Schöier et al. 2002). The vibrationally excited state of HCN has only been observed towards A (Huang et al. 2005). Source A was checked for $\mathrm{D}^{13} \mathrm{CN}$ emission, but not detected (Wampfler et al. 2014). $\mathrm{HCN}$, $\mathrm{H}^{13} \mathrm{CN}$, and DCN have also been observed on envelope scales in the infrared with the Herschel/HIFI guaranteed time CHESS key program (Ceccarelli et al. 2010; Rice et al. 2018). Observations at spatial resolution superseding that of PILS show that $\mathrm{H}^{13} \mathrm{CN}$ and $\mathrm{HC}^{15} \mathrm{~N}$ display inverse $\mathrm{P}$ Cygni profiles near source $\mathrm{B}$, indicating infall, a small velocity gradient and an almost face-on orientation for the B-disc (Zapata et al. 2013, who analysed the lines labelled in Baryshev et al. 2015). HCN and DCN are covered in the data of Lis et al. (2016), but, unfortunately, are not discussed towards the position of IRAS 16293-2422. The works of Zapata et al. (2013); Wampfler et al. (2014) targeted too few lines to estimate column densities. The latest derivation of the column density is presented in Appendix A, which has also been mentioned in Rice et al. (2018).

\section{C2.3 Hydrogen isocyanide $(H N C), \mathrm{m}=27$}

$\mathrm{HNC}, \mathrm{HN}^{13} \mathrm{C}$, and DNC have been detected on smaller envelope scales (van Dishoeck et al. 1995; Caux et al. 2011) at abundances that are reproducible with jump profiles (Schöier et al. 2002). $\mathrm{H}^{15} \mathrm{NC}$ was additionally detected towards source A (Wampfler et al. 2014). HNC was detected on envelope scales in the infrared with the Herschel/HIFI guaranteed time CHESS key program (Ceccarelli et al. 2010). HNC and DNC are covered in the data of Lis et al. (2016), but, unfortunately, are not discussed towards the position of IRAS 16293-2422. The works of Caux et al. (2011); Wampfler et al. (2014) targeted too few lines to estimate column densities. The latest estimate of the column density is presented in Appendix A.

\section{C2.4 Nitroxyl/Azanone/Nitrosyl hydride (HNO), $\mathrm{m}=31$}

A tentative detection of DNO was claimed in absorption towards source A (Chandler et al. 2005). Coutens et al. (2019a) derived an upper limit for HNO towards source B.

\section{C2.5 Cyanic acid (HOCN), $\mathrm{m}=43$}

Marcelino et al. (2010) claimed a tentative detection of HOCN. This was not confirmed by Ligterink et al. (2017).

C2.6 Fulminic acid/Oxidoazaniumylidynemethane (HCNO), $\mathrm{m}=$ 43

Marcelino et al. (2010) reported a non-detection of HCNO.

\section{C2.7 Isocyanic acid $(H N C O), \mathrm{m}=43$}

HNCO has been detected on the envelope scales (van Dishoeck et al. 1995; Caux et al. 2011) and towards both protostars individually (Bisschop et al. 2008; Jørgensen et al. 2011). An upper limit for $\mathrm{H}^{15} \mathrm{NCO}$ has been derived (van Dishoeck et al. 1995). $\mathrm{HNC}{ }^{18} \mathrm{O}$ was detected towards both, A and B (Jørgensen et al. 2011). A combined study of IRAM $30 \mathrm{~m}$ TIMASSS, APEX, Herschel/HIFI CHESS and ALMA PILS data has been carried out by Hernández-Gómez et al. (2019b) to infer column densities of HNCO for different components of the system. The authors argued that the emission in their observations is dominated by the nine times brighter source A; hence, the presented values are thought to be representative of the large-scale structures encompassing A (Section 2.4 of HernándezGómez et al. 2019b). Coutens et al. (2016); Ligterink et al. (2017) detected DNCO and $\mathrm{HN}^{13} \mathrm{CO}$ towards source B and derived accurate column densities for them, and derived upper limits for $\mathrm{H}^{15} \mathrm{NCO}$ and $\mathrm{HNC}^{18} \mathrm{O}$. Martín-Doménech et al. (2017) detected $\mathrm{HNC}^{18} \mathrm{O}$ towards source $\mathrm{B}$ and consequently, also derived a column density of HNCO. Manigand et al. submitted detected HNCO and DNCO towards source A and derived accurate column densities.

\section{C2.8 Methyl cyanide/Acetonitrile $\left(\mathrm{CH}_{3} \mathrm{CN}\right), \mathrm{m}=41$}

$\mathrm{CH}_{3} \mathrm{CN}$ has been observed on envelope scales (van Dishoeck et al. 1995; Caux et al. 2011; Andron et al. 2018) and then individually towards both protostars (Cazaux et al. 2003; Bottinelli et al. 2004; Bisschop et al. 2008; Jørgensen et al. 2011). $\mathrm{CH}_{2} \mathrm{DCN}$ has been labelled in fig. 1 of Parise et al. (2004) of envelope-scale emission and detected towards both protostars by Jørgensen et al. (2011). $\mathrm{CH}_{3}^{13} \mathrm{CN}$ was detected towards source B by Bisschop et al. (2008), and then towards both (Jørgensen et al. 2011). Calcutt et al. (2018b) detected methyl cyanide and five of its isotopologues $\left({ }^{13} \mathrm{CH}_{3} \mathrm{CN}, \mathrm{CH}_{3}^{13} \mathrm{CN}, \mathrm{CH}_{3} \mathrm{C}^{15} \mathrm{~N}, \mathrm{CH}_{2} \mathrm{DCN}\right.$, and $\left.\mathrm{CHD}_{2} \mathrm{CN}\right)$ towards both sources $\mathrm{A}$ and $\mathrm{B}$, and derived accurate column densities. 


\section{C2.9 Vinyl cyanide/Acrylonitrile $\left(\mathrm{C}_{2} \mathrm{H}_{3} \mathrm{CN}\right), \mathrm{m}=53$}

$\mathrm{C}_{2} \mathrm{H}_{3} \mathrm{CN}$ has been firmly detected, and $\mathrm{CH}_{2} \mathrm{CDCN}$ tentatively, towards the two protostars individually (Kuan et al. 2004; Huang et al. 2005). Calcutt et al. (2018b) re-confirmed the detection of vinyl cyanide towards source B and derived an accurate column density, but only were able to derive an upper limit towards source A.

\section{C2.10 Ethyl cyanide/Propionitrile $\left(\mathrm{C}_{2} \mathrm{H}_{5} \mathrm{CN}\right), \mathrm{m}=55$}

$\mathrm{C}_{2} \mathrm{H}_{5} \mathrm{CN}$ was initially detected only towards source B (Cazaux et al. 2003; Huang et al. 2005), but then quickly towards both (Remijan \& Hollis 2006; Jørgensen et al. 2011). Subsequent observations with the Combined Array for Research in Millimeterwave Astronomy (CARMA) yielded only upper limits (Shiao et al. 2010). Observations on envelope scales have also only yielded upper limits (Remijan et al. 2003). Ethyl cyanide was re-confirmed towards both sources by Calcutt et al. (2018b) with accurate column densities derived.

\section{C2.11 Formamide $\left(\mathrm{NH}_{2} \mathrm{CHO}\right), \mathrm{m}=45$}

First detection was secured with TIMASSS in conjunction with newer IRAM $30 \mathrm{~m}$ data on envelope scales (Kahane et al. 2013). Coutens et al. (2016) detected $\mathrm{NH}_{2} \mathrm{CHO}$ in the $v=0$ and $v_{12}=$ 1 states, $\mathrm{NH}_{2} \mathrm{CDO}$, cis-NHDCHO, trans-NHDCHO, $\mathrm{NH}_{2}^{13} \mathrm{CHO}$ towards source $\mathrm{B}$ and computed accurate column densities, as well as derived upper limits for ${ }^{15} \mathrm{NH}_{2} \mathrm{CHO}$ and $\mathrm{NH}_{2} \mathrm{CH}^{18} \mathrm{O}$. Manigand et al. submitted detected $\mathrm{NH}_{2} \mathrm{CHO}$ towards source $\mathrm{A}$ and derived an accurate column density.

\section{C2.12 Methyl isocyanide $\left(\mathrm{CH}_{3} \mathrm{NC}\right), \mathrm{m}=41$}

Calcutt et al. (2018a) detected $\mathrm{CH}_{3} \mathrm{NC}$ towards source $\mathrm{B}$ and computed an accurate column density, and derived an upper limit towards source A.

\section{C2.13 Methyl isocyanate $\left(\mathrm{CH}_{3} \mathrm{NCO}\right), \mathrm{m}=57$}

Methyl isocyanate was independently detected by Ligterink et al. (2017) and Martín-Doménech et al. (2017) towards source B, and towards source A by Ligterink et al. (2017).

\section{C2.14 Acetonitrile oxide $\left(\mathrm{CH}_{3} \mathrm{CNO}\right), \mathrm{m}=57$}

$\mathrm{CH}_{3} \mathrm{CNO}$ was searched for by Ligterink et al. (2017), but undetected.

\section{C2.15 Methyl Cyanate $\left(\mathrm{CH}_{3} \mathrm{OCN}\right), \mathrm{m}=57$}

$\mathrm{CH}_{3} \mathrm{OCN}$ was searched for by Ligterink et al. (2017), but undetected.

\section{C2.16 Cyanide $(\mathrm{CN}), \mathrm{m}=26$}

CN has been detected on envelope scales (van Dishoeck et al. 1995; Caux et al. 2011).
C2.17 Cyanoacetylene $\left(\mathrm{HC}_{3} \mathrm{~N}\right), \mathrm{m}=51$

$\mathrm{HC}_{3} \mathrm{~N}$ was detected by van Dishoeck et al. (1995), while Caux et al. (2011) additionally detected $\mathrm{DC}_{3} \mathrm{~N}$. Its envelope abundances can be reproduced with jump profiles (Schöier et al. 2002). The emission from $\mathrm{HC}_{3} \mathrm{~N}$ seems to originate from both sources (Kuan et al. 2004; Chandler et al. 2005); however, Jørgensen et al. (2011) were able to secure a detection only towards source $\mathrm{A}$. $\mathrm{HC}_{3} \mathrm{~N}$ and $\mathrm{DC}_{3} \mathrm{~N}$ were observed on envelope scales in TIMASSS (Jaber Al-Edhari et al. 2017). Calcutt et al. (2018b) firmly detected $\mathrm{HC}_{3} \mathrm{~N}$ towards both protostars and derived accurate column densities.

\section{C2.18 Cyanobutadiyne $\left(\mathrm{HC}_{5} \mathrm{~N}\right), \mathrm{m}=75$}

$\mathrm{HC}_{5} \mathrm{~N}$ has been detected on envelope scales in TIMASSS (Jaber Al-Edhari et al. 2017).

\section{C2.19 Methylene amidogen $\left(\mathrm{H}_{2} \mathrm{CN}\right), \mathrm{m}=28$}

$\mathrm{H}_{2} \mathrm{CN}$ has been detected towards both protostars (Jørgensen et al. 2011).

\section{C2.20 Nitric oxide $(\mathrm{NO}), \mathrm{m}=30$}

NO has been detected on envelope scales (Caux et al. 2011), and towards source B (Ligterink et al. 2018a) with an accurate column density provided.

\section{C2.21 Nitrous oxide $\left(\mathrm{N}_{2} \mathrm{O}\right), \mathrm{m}=44$}

$\mathrm{N}_{2} \mathrm{O}$ has been detected towards source B by Ligterink et al. (2018a) with an accurate column density provided.

\section{C2.22 Hydroxylamine $\left(\mathrm{NH}_{2} \mathrm{OH}\right), \mathrm{m}=33$}

An upper limit for $\mathrm{NH}_{2} \mathrm{OH}$ towards source $\mathrm{B}$ has been derived by Ligterink et al. (2018a).

\section{C2.23 Methanimine $\left(\mathrm{CH}_{2} \mathrm{NH}\right), \mathrm{m}=29$}

$\mathrm{CH}_{2} \mathrm{NH}$ has been detected towards source B by Ligterink et al. (2018a) with an accurate column density provided.

\section{C2.24 Methylamine $\left(\mathrm{CH}_{3} \mathrm{NH}_{2}\right), \mathrm{m}=31$}

An upper limit for $\mathrm{CH}_{3} \mathrm{NH}_{2}$ towards source $\mathrm{B}$ has been derived by Ligterink et al. (2018a).

\section{C2.25 Acetamide $\left(\mathrm{CH}_{3} \mathrm{C}(\mathrm{O}) \mathrm{NH}_{2}\right), \mathrm{m}=59$}

Acetamide has been tentatively detected towards source B by Ligterink et al. (2018b).

\section{C2.26 N-methylformamide $\left(\mathrm{CH}_{3} \mathrm{NHCHO}\right), \mathrm{m}=59$}

An upper limit for $\mathrm{N}$-methylformamide has been derived towards source B by Ligterink et al. (2018b). 


\section{C2.27 Cyanamide $\left(\mathrm{NH}_{2} \mathrm{CN}\right), \mathrm{m}=42$}

$\mathrm{NH}_{2} \mathrm{CN}, \mathrm{NHDCN}$, and $\mathrm{NH}_{2}^{13} \mathrm{CN}$ have been detected towards source B by Coutens et al. (2018) with accurate column densities derived. $\mathrm{H}_{2}^{15} \mathrm{NCN}$ has been tentatively detected, and an upper limit for $\mathrm{H}_{2} \mathrm{NC}^{15} \mathrm{~N}$ has been derived towards source $\mathrm{B}$ (Coutens et al. 2019b).

\section{C2.28 Imidogen $(\mathrm{NH}), \mathrm{m}=15$}

The NH radical has been detected in absorption in the infrared with the Herschel/HIFI guaranteed time CHESS key program (Bacmann et al. 2010; Hily-Blant et al. 2010). The ND radical has also been detected (Bacmann et al. 2010).

\section{C2.29 Amidogen $\left(\mathrm{NH}_{2}\right), \mathrm{m}=16$}

The $\mathrm{NH}_{2}$ radical has been detected in absorption in the infrared with the Herschel/HIFI guaranteed time CHESS key program (HilyBlant et al. 2010).

\section{C2.30 Diazenylium $\left(\mathrm{N}_{2} \mathrm{H}^{+}\right), \mathrm{m}=29$}

$\mathrm{N}_{2} \mathrm{H}^{+}$and $\mathrm{N}_{2} \mathrm{D}^{+}$were detected at radio frequencies on large cloud and core scales, as well as envelope scales (Castets et al. 2001; Lis et al. 2002, 2016). $\mathrm{N}_{2} \mathrm{H}^{+}$was also detected on envelope scales in the infrared with the Herschel/HIFI guaranteed time CHESS key program (Ceccarelli et al. 2010). On the scale of individual envelopes, $\mathrm{N}_{2} \mathrm{D}^{+}$is seen only towards A (Jørgensen et al. 2011). Most recently, the distribution of $\mathrm{N}_{2} \mathrm{D}^{+}$has been analysed by Murillo et al. (2018b) on the scale of the individual protostars and the circumbinary envelope.

\section{C2.31 Glycolonitrile $\left(\mathrm{HOCH}_{2} \mathrm{CN}\right), \mathrm{m}=57$}

Glycolonitrile was detected towards source B by Zeng et al. (2019).

\section{C2.32 Aminoacetonitrile $\left(\mathrm{NH}_{2} \mathrm{CH}_{2} \mathrm{CN}\right), \mathrm{m}=56$}

An upper limit was derived towards source B by Zeng et al. (2019).

\section{C2.33 Formyl cyanide $($ CHOCN), $\mathrm{m}=55$}

An upper limit was derived towards source B by Zeng et al. (2019).

\section{C2.34 Ethenimine $\left(\mathrm{CH}_{2} \mathrm{CNH}\right), \mathrm{m}=41$}

An upper limit was derived towards source B by Zeng et al. (2019).

\section{C2.35 Nitrous acid (HONO), $\mathrm{m}=47$}

Coutens et al. (2019a) detected HONO towards source B and derived an accurate column density.

\section{C2.36 Nitrogen dioxide $\left(\mathrm{NO}_{2}\right), \mathrm{m}=46$}

Coutens et al. (2019a) derived an upper limit for $\mathrm{NO}_{2}$ towards source B.
C2.37 Nitrosyl cation $\left(\mathrm{NO}^{+}\right), \mathrm{m}=30$

Coutens et al. (2019a) derived an upper limit for $\mathrm{NO}^{+}$towards source B.

C2.38 Nitric acid $\left(\mathrm{HNO}_{3}\right), \mathrm{m}=63$

Coutens et al. (2019a) derived an upper limit for $\mathrm{HNO}_{3}$ towards source B.

\section{C3 S-bearing molecules}

C3.1 Hydrogen sulphide $\left(\mathrm{H}_{2} \mathrm{~S}\right), \mathrm{m}=34$

$\mathrm{H}_{2} \mathrm{~S}$ was detected on envelope scales (Blake et al. 1994), as was HDS (van Dishoeck et al. 1995; Caux et al. 2011). It was also observed towards the two protostars individually (Chandler et al. 2005; Jørgensen et al. 2011). On large cloud and core scales, $\mathrm{H}_{2} \mathrm{~S}$ is also observed, but at lower abundances than closer to the binary source (Wakelam et al. 2004). $\mathrm{H}_{2} \mathrm{~S}$ was detected on envelope scales in the infrared with the Herschel/HIFI guaranteed time CHESS key program (Ceccarelli et al. 2010). Baryshev et al. (2015) reported a line of $\mathrm{H}_{2} \mathrm{~S}$ in absorption from the foreground cloud. HDS and $\mathrm{HD}^{34} \mathrm{~S}$ were detected towards source B by Drozdovskaya et al. (2018) with accurate column densities provided.

\section{C3.2 Carbon monosulphide (CS), $\mathrm{m}=44$}

$\mathrm{C}^{32} \mathrm{~S}$ was the second molecule studied in IRAS 16293-2422. Its emission line profile showed strong self-absorption and prominent asymmetry, which was interpreted as a signature of infall (Walker et al. 1986; van Dishoeck et al. 1995). $\mathrm{C}^{34} \mathrm{~S}$ was observed soon thereafter (Menten et al. 1987). CS, like CO, is present on the large cloud and core scales; and the smaller circumbinary envelope and disc-A and -B scales (Mizuno et al. 1990; Walker, Carlstrom \& Bieging 1993; Blake et al. 1994; Narayanan et al. 1998; Caux et al. 2011; Jørgensen et al. 2011; Takakuwa \& Kamazaki 2011; van der Wiel et al. 2019). It also traces the outflows (Walker et al. 1990; Girart et al. 2014), and shocks, as solidified by the detection of vibrationally excited lines (Blake et al. 1994; Walker, Maloney \& Serabyn 1994). Its envelope abundance has also been modelled with jump profiles (Schöier et al. 2002). ${ }^{13} \mathrm{CS}, \mathrm{C}^{33} \mathrm{~S}$, and $\mathrm{C}^{34} \mathrm{~S}$ were detected on smaller envelope scales (Caux et al. 2011; Jørgensen et al. 2011; Favre et al. 2014). CS and $C^{34} S$ were detected on envelope scales in the infrared with the Herschel/HIFI guaranteed time CHESS key program (Ceccarelli et al. 2010). $C^{34} S, C^{33} S$, and $\mathrm{C}^{36} \mathrm{~S}$ were detected towards source B by Drozdovskaya et al. (2018) with accurate column densities derived.

C3.3 Acetylene sulphide $\left(C_{2} S\right), \mathrm{m}=56$

$\mathrm{C}_{2} \mathrm{~S}$ has been detected on envelope scales (Caux et al. 2011).

\section{C3.4 Carbonyl sulphide (OCS), $\mathrm{m}=60$}

OCS, $\mathrm{OC}^{34} \mathrm{~S}, \mathrm{O}^{13} \mathrm{CS}$, and ${ }^{18} \mathrm{OCS}$ have been detected on envelope scales (Blake et al. 1994; van Dishoeck et al. 1995; Huang et al. 2005; Caux et al. 2011). OCS has subsequently been detected towards both protostars individually, but $\mathrm{O}^{13} \mathrm{CS}$ only towards $\mathrm{A}$ (Jørgensen et al. 2011). Additionally, OCS has been argued to display abundance enhancements at the centrifugal barrier in both sources (Oya et al. 2016, 2018); however, these studies claim 
optically thin emission, which is likely not the case as analysed in Drozdovskaya et al. (2018). OCS in its ground and vibrationally excited states, as well as $\mathrm{O}^{13} \mathrm{CS}, \mathrm{OC}^{34} \mathrm{~S}, \mathrm{OC}^{33} \mathrm{~S}$, and ${ }^{18} \mathrm{OCS}$ were detected towards source B by Drozdovskaya et al. (2018) with accurate column densities derived.

\section{C3.5 Sulphur monoxide ( $\mathrm{SO}$ ), $\mathrm{m}=48$}

Initially, SO was only detected towards source A and proposed to be associated with outflows; while its non-detection towards source B indicated an abundance at least a factor of 10 lower in comparison (Mundy et al. 1992). The detection towards A was verified several times (Chandler et al. 2005; Huang et al. 2005). SO and its vibrationally excited state, ${ }^{34} \mathrm{SO}$ and $\mathrm{S}^{18} \mathrm{O}$ were observed on circumbinary envelope scales (Blake et al. 1994; Wakelam et al. 2004; Caux et al. 2011). SO was detected on envelope scales in the infrared with the Herschel/HIFI guaranteed time CHESS key program (Ceccarelli et al. 2010). SO was finally detected towards both protostars, but ${ }^{33} \mathrm{SO}$ and ${ }^{34} \mathrm{SO}$ only towards A (Jørgensen et al. 2011). SO is labelled in Baryshev et al. (2015) for source B. Lindberg et al. (2017) also demonstrated its presence on the large cloud scales. The presence of SO was re-confirmed for source B by Drozdovskaya et al. (2018) with an accurate column density derived.

\section{C3.6 Sulphur dioxide $\left(\mathrm{SO}_{2}\right), \mathrm{m}=64$}

$\mathrm{SO}_{2}$ has been detected on envelope scales (Blake et al. 1994; van Dishoeck et al. 1995; Caux et al. 2011) and large cloud and core scales (Wakelam et al. 2004) at lower abundances. Its vibrationally excited state was detected towards both inner regions of sources $\mathrm{A}$ and $\mathrm{B}$, but ${ }^{34} \mathrm{SO}_{2}$ was only observed towards A (Chandler et al. 2005; Huang et al. 2005). $\mathrm{SO}_{2}$ was detected on envelope scales in the infrared with the Herschel/HIFI guaranteed time CHESS key program (Ceccarelli et al. 2010). Subsequently, $\mathrm{SO}_{2}$ was observed towards both protostars, but ${ }^{33} \mathrm{SO}_{2},{ }^{34} \mathrm{SO}_{2}, \mathrm{SO}^{18} \mathrm{O}$, and $\mathrm{SO}^{17} \mathrm{O}$ only towards A (Jørgensen et al. 2011). ${ }^{33} \mathrm{SO}_{2},{ }^{34} \mathrm{SO}_{2}$ are labelled in Baryshev et al. (2015) for source B. The presence of $\mathrm{SO}_{2}$ and ${ }^{34} \mathrm{SO}_{2}$ were re-confirmed for source B by Drozdovskaya et al. (2018) with accurate column densities derived.

\section{C3.7 Thioformaldehyde $\left(\mathrm{H}_{2} \mathrm{CS}\right), \mathrm{m}=46$}

$\mathrm{H}_{2} \mathrm{CS}, \mathrm{H}_{2}^{13} \mathrm{CS}, \mathrm{H}_{2} \mathrm{C}^{34} \mathrm{~S}$, and HDCS have been detected on envelope scales (Blake et al. 1994; Huang et al. 2005; Caux et al. 2011). Towards the two protostars individually, $\mathrm{H}_{2} \mathrm{CS}$ and HDCS have been observed, but $\mathrm{H}_{2} \mathrm{C}^{34} \mathrm{~S}$ and $\mathrm{D}_{2} \mathrm{CS}$ only towards A (Jørgensen et al. 2011). $\mathrm{H}_{2} \mathrm{CS}$ has been argued to trace the envelope and the A- and B-discs (Oya et al. 2016, 2018), which collaborates nearly optically thin emission claimed by Drozdovskaya et al. (2018). The presence of $\mathrm{H}_{2} \mathrm{CS}$ and HDCS were re-confirmed for source B by Drozdovskaya et al. (2018) with accurate column densities derived, and upper limits derived for $\mathrm{H}_{2} \mathrm{C}^{34} \mathrm{~S}$ and $\mathrm{D}_{2} \mathrm{CS}$.

\section{C3.8 Methyl mercaptan/Methanethiol $\left(\mathrm{CH}_{3} \mathrm{SH}\right), \mathrm{m}=48$}

$\mathrm{CH}_{3} \mathrm{SH}$ has been detected on circumbinary envelope scales (Majumdar et al. 2016) and towards source B (Drozdovskaya et al. 2018) with an accurate column density derived. An upper limits for $\mathrm{CH}_{3} \mathrm{SD}$ has been derived towards source B (Zakharenko et al. 2019).

\section{C3.9 Ethyl mercaptan/Ethanethiol $\left(\mathrm{C}_{2} \mathrm{H}_{5} \mathrm{SH}\right), \mathrm{m}=62$}

Upper limits for gauche- and anti- $\mathrm{C}_{2} \mathrm{H}_{5} \mathrm{SH}$, and gauche- $\mathrm{C}_{2} \mathrm{H}_{5}^{34} \mathrm{SH}$, were derived towards source B by Drozdovskaya et al. (2018).

\section{C3.10 Disulphur $\left(S_{2}\right), \mathrm{m}=64$}

An upper limit for $S_{2}$ on the envelope scale has been reported based on APEX data by Martín-Doménech et al. (2016). An upper limit has also been derived towards source B by Drozdovskaya et al. (2018).

\section{C3.11 Trisulphur $\left(S_{3}\right), \mathrm{m}=96$}

An upper limit has been derived towards source B by Drozdovskaya et al. (2018).

\section{C3.12 Tetrathietane $\left(S_{4}\right), \mathrm{m}=128$}

An upper limit has been derived towards source B by Drozdovskaya et al. (2018).

\section{C3.13 Disulphanide $\left(H S_{2}\right), \mathrm{m}=65$}

An upper limit for $\mathrm{HS}_{2}$ on the envelope scale has been reported based on APEX data by Martín-Doménech et al. (2016). An upper limit has also been derived towards source B by Drozdovskaya et al. (2018).

\section{C3.14 Disulphane $\left(\mathrm{H}_{2} \mathrm{~S}_{2}\right), \mathrm{m}=66$}

An upper limit for $\mathrm{H}_{2} \mathrm{~S}_{2}$ on the envelope scale has been reported based on APEX data by Martín-Doménech et al. (2016). An upper limit has also been derived towards source B by Drozdovskaya et al. (2018).

\section{C3.15 Sulphur mononitride (NS), $\mathrm{m}=46$}

NS is detected towards source A (Chandler et al. 2005) and on envelope scales (Caux et al. 2011). An upper limit was derived towards source B by Drozdovskaya et al. (2018).

\section{C3.16 Thioformyl (HCS), $\mathrm{m}=45$}

HCS has been detected on envelope scales (Blake et al. 1994; van Dishoeck et al. 1995; Caux et al. 2011), also solely towards source A (Jørgensen et al. 2011). An upper limit has been derived towards source B by Drozdovskaya et al. (2018).

\section{C3.17 Thioformylium $\left(\mathrm{HCS}^{+}\right), \mathrm{m}=45$}

$\mathrm{DCS}^{+}$has been tentatively detected towards source A (Jørgensen et al. 2011). Upper limits for $\mathrm{HCS}^{+}, \mathrm{DCS}^{+}$, and $\mathrm{HC}^{34} \mathrm{~S}^{+}$have been derived towards source B by Drozdovskaya et al. (2018).

\section{C3.18 Disulphur monoxide $\left(\mathrm{S}_{2} \mathrm{O}\right), \mathrm{m}=80$}

An upper limit for $\mathrm{S}_{2} \mathrm{O}$ was derived towards source B by Drozdovskaya et al. (2018). 
C3.19 Disulphur dioxide $\left(\mathrm{S}_{2} \mathrm{O}_{2}\right), \mathrm{m}=96$

An upper limit for cis- $\mathrm{S}_{2} \mathrm{O}_{2}$ was derived towards source $\mathrm{B}$ by Drozdovskaya et al. (2018).

\section{C3.20 Thiohydroxymethylidyne (HSC), $\mathrm{m}=45$}

An upper limit for HSC was derived towards source B by Drozdovskaya et al. (2018).

\section{C3.21 Thioethenylidene (CCS), $\mathrm{m}=56$}

An upper limit for CCS was derived towards source B by Drozdovskaya et al. (2018).

\section{C3.22 Thioketene $\left(\mathrm{H}_{2} \mathrm{C}_{2} \mathrm{~S}\right), \mathrm{m}=58$}

An upper limit for $\mathrm{H}_{2} \mathrm{C}_{2} \mathrm{~S}$ was derived towards source $\mathrm{B}$ by Drozdovskaya et al. (2018).

$\mathrm{C} 3.23 \mathrm{c}-\mathrm{C}_{2} \mathrm{H}_{4} \mathrm{~S}, \mathrm{~m}=60$

An upper limit for $\mathrm{c}-\mathrm{C}_{2} \mathrm{H}_{4} \mathrm{~S}$ was derived towards source $\mathrm{B}$ by Drozdovskaya et al. (2018).

C3.24 Thiocyanate (NCS), $\mathrm{m}=58$

An upper limit for NCS was derived towards source B by Drozdovskaya et al. (2018).

\section{C3.25 Isothiocyanic acid (HNCS), $\mathrm{m}=59$}

An upper limit for a-type HNCS was derived towards source B by Drozdovskaya et al. (2018).

\section{C3.26 Thiocyanic acid (HSCN), $\mathrm{m}=59$}

An upper limit for HSCN was derived towards source B by Drozdovskaya et al. (2018).

\section{C4 Other species}

C4.1 Hydrogen chloride $(\mathrm{HCl}), \mathrm{m}=36$

$\mathrm{HCl}$ and $\mathrm{H}^{37} \mathrm{Cl}$ were detected on envelope scales in the infrared with the Herschel/HIFI guaranteed time CHESS key program (Ceccarelli et al. 2010) and with the CSO (Peng et al. 2010).

\section{C4.2 Methyl chloride $\left(\mathrm{CH}_{3} \mathrm{Cl}\right), \mathrm{m}=50$}

$\mathrm{CH}_{3} \mathrm{Cl}$ and $\mathrm{CH}_{3}^{37} \mathrm{Cl}$ were detected towards source $\mathrm{B}$ by Fayolle et al. (2017) with accurate column densities derived.

\section{C4.3 Fluoromethane $\left(\mathrm{CH}_{3} \mathrm{~F}\right), \mathrm{m}=34$}

An upper limit for $\mathrm{CH}_{3} \mathrm{~F}$ was derived towards source B by Fayolle et al. (2017).
C4.4 Silicon monoxide ( $\mathrm{SiO}), \mathrm{m}=44$

$\mathrm{SiO}$ and ${ }^{29} \mathrm{SiO}$ were detected on envelope scales (Blake et al. 1994; Ceccarelli et al. 2000a; Caux et al. 2011) at abundances reproducible with jump profiles (Schöier et al. 2002). On large cloud and core scales, $\mathrm{SiO}$ emission seems to arise from the interaction of the outflowing gas and the dense ambient clump material, the outflow lobe itself, and the central region of the lobe (Hirano et al. 2001; Wakelam et al. 2004). It has been claimed to be consistent with Ctype shock profiles and estimated to be enhanced by a factor of $\sim 330$ relative to the ambient gas (Garay et al. 2002; Girart et al. 2014). SiO has been suggested to be massing at an off-source position between the two protostars (Remijan \& Hollis 2006; Rao et al. 2009). SiO has been observed towards the two protostars individually by Jørgensen et al. (2011) and van der Wiel et al. (2019). Oya et al. (2018) address $\mathrm{SiO}$ emission near source $\mathrm{B}$ as a tracer of the outflow launch, but could not rule out the influence of A on B.

C4.5 Silicon monosulphide (SiS), $\mathrm{m}=60$

SiS and ${ }^{29} \mathrm{SiS}$ were detected on envelope scales (Blake et al. 1994). $\mathrm{Si}^{34} \mathrm{~S}$ was tentatively detected towards source A (Chandler et al. 2005).

\section{C4.6 Phosphorus mononitride (PN), $\mathrm{m}=45$}

A line of PN has been labelled on envelope scales (Caux et al. 2011). The latest derivation of an upper limit on the column density is presented in Appendix A.

\section{C4.7 Trihydrogen $\left(H_{3}^{+}\right), \mathrm{m}=3$}

On cloud scales, $\mathrm{H}_{2} \mathrm{D}^{+}$has been detected (Stark et al. 2004). Orthoand para- $\mathrm{H}_{2} \mathrm{D}^{+}$were detected with APEX in emission and with SOFIA in absorption, respectively (Brünken et al. 2014). Ortho$\mathrm{D}_{2} \mathrm{H}^{+}$was now also found with SOFIA in absorption (Harju et al. 2017).

\section{C5 Detected atoms}

Several atomic lines have also been detected on circumbinary envelope scales towards this source, which include OI with the Kuiper Airborn Observatory (Ceccarelli et al. 1997), CI with the JCMT (Ceccarelli et al. 1998b), and CII with ISO/LWS (Ceccarelli et al. 1998b).

\section{C6 Hydrocarbons}

C6.1 Methylidyne $(\mathrm{CH}), \mathrm{m}=13$

$\mathrm{CH}$ has been observed in absorption in the infrared with the Herschel/HIFI guaranteed time CHESS key program on the large cloud and circumbinary envelope scales (Bottinelli et al. 2014).

\section{C6.2 Ethynyl $\left(\mathrm{C}_{2} \mathrm{H}\right), \mathrm{m}=25$}

$\mathrm{C}_{2} \mathrm{H}$ and $\mathrm{C}_{2} \mathrm{D}$ have been detected on envelope scales (van Dishoeck et al. 1995; Caux et al. 2011). $\mathrm{C}_{2} \mathrm{H}$ was detected and its distribution studied by Murillo et al. (2018b) and van der Wiel et al. (2019) on the scale of the individual protostars and the circumbinary envelope. 
C6.3 Cyclopropanediylidenyl $\left(c-C_{3} H\right), \mathrm{m}=37$

c- $\mathrm{C}_{3} \mathrm{H}$ has been detected on envelope scales (Caux et al. 2011).

C6.4 Cyclopropenylidene $\left(c-\mathrm{C}_{3} \mathrm{H}_{2}\right), \mathrm{m}=38$

c- $\mathrm{C}_{3} \mathrm{H}_{2}$ has been detected on cloud scales (Lindberg et al. 2017), envelope scales (van Dishoeck et al. 1995; Caux et al. 2011), and on the scales of the individual envelopes (Kuan et al. 2004; Majumdar et al. 2017). An upper limit on $\mathrm{C}_{3} \mathrm{HD}$ was reported by van Dishoeck et al. (1995) and a detection was secured by Caux et al. (2011) on the scales of the circumbinary envelope. On the scales of individual envelopes, $\mathrm{c}-\mathrm{C}_{3} \mathrm{H}_{2}$ and $\mathrm{c}-\mathrm{C}_{3} \mathrm{HD}$ have now also been observed (Majumdar et al. 2017). c- $C_{3} D_{2}$ has been tentatively labelled in Martín-Doménech et al. (2016). Most recently, its distribution has been analysed by Murillo et al. (2018b) on the scale of the individual protostars and the circumbinary envelope.
C6.5 Butadiynyl $\left(\mathrm{C}_{4} \mathrm{H}\right), \mathrm{m}=49$

$\mathrm{C}_{4} \mathrm{H}$ has been detected on envelope scales (Caux et al. 2011).

C6.6 Propyne $\left(\mathrm{CH}_{3} \mathrm{CCH}\right), \mathrm{m}=40$

$\mathrm{CH}_{3} \mathrm{CCH}$ was detected on envelope scales (van Dishoeck et al. 1995; Caux et al. 2011; Andron et al. 2018) and towards source B (Cazaux et al. 2003). $\mathrm{CH}_{2} \mathrm{DCCH}$ has also been observed on envelope scales (Caux et al. 2011). Calcutt et al. submitted have detected and derived accurate column densities of $\mathrm{CH}_{3} \mathrm{CCH}$ towards both protostars, as well as upper limits for $\mathrm{CH}_{3}^{13} \mathrm{CCH},{ }^{13} \mathrm{CH}_{3} \mathrm{CCH}$, $\mathrm{CH}_{3} \mathrm{C}^{13} \mathrm{CH}, \mathrm{CH}_{3} \mathrm{CCD}, \mathrm{CH}_{2} \mathrm{DCCH}$ towards both protostars.

This paper has been typeset from a $\mathrm{T}_{\mathrm{E}} \mathrm{X} / \mathrm{LT} \mathrm{E} \mathrm{X}$ file prepared by the author. 